\title{
Which AGN jets quench star formation in massive galaxies?
}

\author{
Kung-Yi Su, ${ }^{1,2,3 \star}$ Philip F. Hopkins ${ }^{\bullet},{ }^{3}$ Greg L. Bryan ${ }^{\circledR},{ }^{1}$ Rachel S. Somerville, ${ }^{2}$
} Christopher C. Hayward ${ }^{\oplus},{ }^{2}$ Daniel Anglés-Alcázar, ${ }^{2,4}$ Claude-André Faucher-Giguère ${ }^{\oplus}, 5$

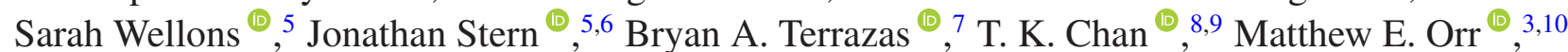
Cameron Hummels, ${ }^{3}$ Robert Feldmann ${ }^{\oplus 11}$ and Dušan Kereš ${ }^{9}$

${ }^{1}$ Department of Astronomy, Columbia University, 550 West 120th Street, New York, NY 10027, USA

${ }^{2}$ Center for Computational Astrophysics, Flatiron Institute, 162 Fifth Avenue, New York, NY 10010, USA

${ }^{3}$ TAPIR 350-17, California Institute of Technology, 1200 E. California Boulevard, Pasadena, CA 91125, USA

${ }^{4}$ Department of Physics, University of Connecticut, 196 Auditorium Road, U-3046, Storrs, CT 06269-3046, USA

${ }^{5}$ Department of Physics \& Astronomy and CIERA, Northwestern University, 1800 Sherman Ave, Evanston, IL 60201, USA

${ }^{6}$ School of Physics \& Astronomy, Tel Aviv University, Tel Aviv 69978, Israel

${ }^{7}$ Harvard-Smithsonian Center for Astrophysics, Cambridge, MA 02138, USA

${ }^{8}$ Institute for Computational Cosmology, Durham University, South Road, Durham DH1 3LE, UK

${ }^{9}$ Department of Physics and Center for Astrophysics and Space Science, University of California at San Diego, 9500 Gilman Drive, La Jolla, CA 92093, USA

${ }^{10}$ Department of Physics and Astronomy, Rutgers, The State University of New Jersey, 136 Frelinghuysen Rd, Piscataway, NJ 08854, USA

${ }^{11}$ Institute for Computational Science, University of Zurich, Winterthurerstrasse 190, CH-8057 Zurich, Switzerland

Accepted 2021 July 10. Received 2021 July 9; in original form 2021 February 3

\begin{abstract}
Without additional heating, radiative cooling of the halo gas of massive galaxies (Milky Way-mass and above) produces cold gas or stars exceeding that observed. Heating from active galactic nucleus (AGN) jets is likely required, but the jet properties remain unclear. This is particularly challenging for galaxy simulations, where the resolution is orders-of-magnitude insufficient to resolve jet formation and evolution. On such scales, the uncertain parameters include the jet energy form [kinetic, thermal, cosmic ray (CR)]; energy, momentum, and mass flux; magnetic fields; opening angle; precession; and duty cycle. We investigate these parameters in a $10^{14} \mathrm{M}_{\odot}$ halo using high-resolution non-cosmological magnetohydrodynamic simulations with the FIRE-2 (Feedback In Realistic Environments) stellar feedback model, conduction, and viscosity. We explore which scenarios qualitatively meet observational constraints on the halo gas and show that CR-dominated jets most efficiently quench the galaxy by providing CR pressure support and modifying the thermal instability. Mildly relativistic $\left(\sim \mathrm{MeV}\right.$ or $\left.\sim 10^{10} \mathrm{~K}\right)$ thermal plasma jets work but require $\sim 10$ times larger energy input. For fixed energy flux, jets with higher specific energy (longer cooling times) quench more effectively. For this halo mass, kinetic jets are inefficient at quenching unless they have wide opening or precession angles. Magnetic fields also matter less except when the magnetic energy flux reaches $\gtrsim 10^{44} \mathrm{erg} \mathrm{s}^{-1}$ in a kinetic jet model, which significantly widens the jet cocoon. The criteria for a successful jet model are an optimal energy flux and a sufficiently wide jet cocoon with a long enough cooling time at the cooling radius.
\end{abstract}

Key words: turbulence - methods: numerical-cosmic rays-galaxies: clusters: intracluster medium-galaxies: jets - galaxies: magnetic fields.

\section{INTRODUCTION}

A major outstanding problem in galaxy formation for decades has been how to 'quench' massive galaxies (stellar masses $\gtrsim 10^{11} \mathrm{M}_{\odot}$ or above $\sim L_{*}$ in the galaxy luminosity function) and keep them 'red and dead' over a large fraction of cosmic time (see e.g. Bell et al. 2003; Kauffmann et al. 2003; Madgwick et al. 2003; Baldry et al. 2004; Blanton et al. 2005; Kereš et al. 2005; Dekel \& Birnboim 2006; Kereš et al. 2009; Pozzetti et al. 2010; Wetzel, Tinker \& Conroy 2012; Feldmann \& Mayer 2015; Voit et al. 2015). The difficulty lies in the classic 'cooling flow' problem - X-ray observations have found

^E-mail: kungyisu@gmail.com significant radiative cooling in the hot gas of elliptical galaxies and clusters, indicating cooling times shorter than a Hubble time (Fabian et al. 1994; Peterson \& Fabian 2006; Stern et al. 2019). However, compared to the inferred cooling flow (reaching up to $\sim 1000 \mathrm{M}_{\odot} \mathrm{yr}^{-1}$ in clusters), neither sufficient cold gas from $\mathrm{HI}$ and $\mathrm{CO}$ observations (McDonald, Veilleux \& Mushotzky 2011; Werner et al. 2013) nor sufficient star formation (Tamura et al. 2001; O'Dea et al. 2008; Rafferty, McNamara \& Nulsen 2008) has been observed in galaxies. Simulations and semi-analytic models which do not suppress the cooling flows, and simply allow gas to cool into the galactic core, typically predict over an order of magnitude higher star formation rates (SFRs) than observed (for recent examples see e.g. the weak/no feedback runs in Sijacki et al. 2007; Somerville et al. 2008; Booth \& Schaye 2009; Choi et al. 2015; Li et al. 2015; Anglés-Alcázar et al. 2017). 
Some heat source or pressure support must be present to compensate for the observed cooling. Moreover, the heating must still preserve the cool core structure (e.g. density and entropy profiles) observed in the majority of galaxies (Peres et al. 1998; Mittal et al. 2009). One way to achieve this is to suppress the cooling flow and maintain a very-low SFR, stable cool-core (CC) cluster. Another possibility is that clusters undergo cool-core-non-cool-core (NCC) cycles: a stronger episode of feedback overturns the cooling flows, resulting in a NCC cluster that gradually recovers to a CC cluster and starts another episode of feedback.

The various non-active galactic nucleus (non-AGN) solutions to the cooling flow problem proposed in the literature generally belong to the former case, including: stellar feedback from shock-heated asymptotic giant branch (AGB) winds (Conroy, van Dokkum \& Kravtsov 2015), Type Ia supernovae (SNe; e.g. Sharma et al. 2012, and references therein), SNe-injected cosmic rays (CRs; Pfrommer et al. 2017; Ruszkowski, Yang \& Zweibel 2017a; Butsky \& Quinn 2018; Farber et al. 2018; Jacob et al. 2018), magnetic fields (Soker \& Sarazin 1990; Beck et al. 1996, 2012), and thermal conduction (Binney \& Cowie 1981; Tucker \& Rosner 1983; Fabian, Voigt \& Morris 2002; Voigt et al. 2002; Zakamska \& Narayan 2003) in the circum-galactic medium (CGM) or intra-cluster medium (ICM), or 'morphological quenching' via altering the galaxy morphology and gravitational stability properties (Dekel, Sari \& Ceverino 2009; Martig et al. 2009). Although these processes can slightly suppress star formation, or help suppress the cooling flows, most previous studies, including our own exhaustive survey studying each of these in simulations similar to those presented here (Su et al. 2019, hereafter Paper I), have shown that they do not fundamentally alter the classic cooling flow picture. In the end, the star formation is still regulated by cooling flows, and the SFR is orders of magnitude too high.

Consequently, AGN feedback seems to be the most promising candidate to solve the cooling flow problem, and there has been a tremendous amount of theoretical work on the topic (for recent studies, see the reference in later paragraphs for the AGN jet and e.g. Eisenreich et al. 2017; Gaspari \& Sagdowski 2017; Li et al. 2018; Pellegrini et al. 2018; Weinberger et al. 2018; Yoon et al. 2018 for other type of AGN feedback; also see e.g. Silk \& Rees 1998; Fabian 1999; Ciotti \& Ostriker 2001; Hopkins et al. 2005, 2006a; Croton et al. 2006; Ciotti, Ostriker \& Proga 2009; Choi et al. 2012 for earlier works). Observational studies also infer that the available energy budget from AGN can match the cooling rate (Bîrzan et al. 2004). There are also observations of un-ambiguous cases of AGN expelling gas from galaxies, injecting thermal energy via shocks or sound waves, or via photo-ionization and Compton heating, or via 'stirring' the CGM and ICM, and creating 'bubbles' of hot plasma with nonnegligible relativistic components that are ubiquitous around massive galaxies (see e.g. Fabian 2012; Hickox \& Alexander 2018 for a detailed review).

However, despite its plausibility and the extensive work above, the detailed physics of AGN feedback remain uncertain, as do the relevant 'input parameters.' Several studies also suggested certain categories of AGN feedback models struggle to stably quench the star formation, self-regulate themselves, or meet some of the observational constraints (e.g. Bîrzan et al. 2004; Vernaleo \& Reynolds 2006; Glines, O'Shea \& Voit 2020; Su et al. 2020). Therefore, a broad systematic exploration of AGN feedback models can be useful to understand which, if any, are more plausible for solving the cooling flow problem. In Su et al. (2020; here after Paper II), we explored various idealized AGN 'toy models' with energy injection in different forms (e.g. direct isotropic momentum injection, turbulent stirring, thermal heating, CR injection). We found that turbulent stirring within a radius of order the halo scale radius, or CR injection (with appropriate energetics) were able to maintain a stable, CC, low-SFR halo for extended periods, across haloes with mass $10^{12}-10^{14} \mathrm{M}_{\odot}$, without obviously violating observational constraints on halo gas properties or exceeding plausible energy budgets for low luminosity AGN in massive galaxies. But in that study, we did not attempt to model realistic jets or AGN outflows; instead, we intentionally considered energy input or 'stirring' rates distributed according to an arbitrary spatial kernel, without considering how that energy would actually propagate from a collimated geometry, or how turbulence would actually be produced. Given that AGN jets can be a dominant source of CRs and an important mechanism to stir turbulence in the CGM, we move a step forward in this work to study the effects of a wide range of more realistic jet models in cooling flows.

Extensive studies have shown that various AGN jet models are, in principle, capable of quenching a galaxy and stopping the cooling flows in galaxy-scale simulations (e.g. Dubois et al. 2010; Gaspari, Brighenti \& Temi 2012a; Yang, Sutter \& Ricker 2012; Li \& Bryan 2014a; Li et al. 2015; Prasad, Sharma \& Babul 2015; Yang \& Reynolds 2016a; Bourne \& Sijacki 2017; Ruszkowski et al. 2017a). However, in such simulations, AGN jets are launched from the smallest resolved scale, acting as a sort of inner boundary condition, instead of being generated self-consistently. Due to the uncertainties of the jet properties at these scales, the details of how the jet is launched are highly model-dependent, spanning a vast parameter space.

AGN jets most likely physically consist of relativistic particles at the black hole horizon scale, powered by magnetic fields through the Blandford-Znajek process (Blandford \& Znajek 1977; Tchekhovskoy, Narayan \& McKinney 2011; Blandford, Meier \& Readhead 2019), where the magnetic energy is supplied by the black hole spin. Recent developments in general-relativistic magnetohydrodynamic (GRMHD) simulations have made it possible to self-consistently follow the formation and evolution of the jet in simulations resolving the black hole horizon scale and accretion disc (e.g. Hawley \& Villiers 2004; Tchekhovskoy et al. 2011; McKinney, Tchekhovskoy \& Bland ford 2012; White, Stone \& Quataert 2019), and the fields carried with the jet can reach $\sim$ Gauss at scales $\ll 1 \mathrm{pc}$ (Guan, Li \& Li 2014). However, at the finest resolvable scale ( $\gtrsim 10 \mathrm{pc}$ ) in galaxy simulations, the jet velocity and magnetic field strength evolve radically through interactions with the surrounding gas. Depending on the model and the sub-resolution environment around the black hole, part of the kinetic energy can be transformed into thermal or CR energy. The balance between thermal, kinetic, magnetic, and CR energy at the scales where jets begin to interact with resolvable galaxy scales (the key for quenching models) therefore remains highly uncertain.

Momentum and kinetic energy can be directly transferred to the gas, suppressing inflows. The fast-moving jets can also shock heat the surrounding gas. Many models have invoked kinetic jets to suppress cooling flows and SFRs in massive haloes (e.g. Dubois et al. 2010; Gaspari et al. 2012a; Li \& Bryan 2014a; Prasad et al. 2015; Yang \& Reynolds 2016a). Many models in the literature also invoke the idea that AGN can effectively drive strong pressure-driven outflows and offset cooling if a large fraction of the accretion energy is thermalized (Begelman 2004; Springel, Di Matteo \& Hernquist 2005; Di Matteo, Springel \& Hernquist 2005; Hopkins et al. 2006a, b, 2007, 2008; Johansson, Naab \& Burkert 2009; Hopkins \& Elvis 2010; Ostriker et al. 2010; Faucher-Giguère \& Quataert 2012; Dubois et al. 2013; Barai et al. 2014; Weinberger et al. 2017a; Pillepich et al. 2018; Richings \& Faucher-Giguère 2018a, b; Torrey et al. 2020). Physically, as the jet propagates, part of the kinetic energy can thermalize through shocks. Some studies have argued that the 
heat from those weak shocks can suppress cooling flows and SFRs in massive haloes (Yang \& Reynolds 2016b; Li, Ruszkowski \& Bryan 2017; Martizzi et al. 2019). The magnetic fields carried by the jet at its launch might also help suppress cooling flows by providing additional pressure support (Soker \& Sarazin 1990; Beck et al. 1996, 2012), although our studies find that they have limited effects on global star formation properties of sub- $L_{*}$ galaxies ( $\mathrm{Su}$ et al. 2017). ${ }^{1}$ Finally, CRs arise generically from processes that occur in fast shocks, so they could come from shocked winds or outflows. But they are particularly associated with relativistic jets from AGN (where they can make up the bulk of the jet energy; Berezinsky, Gazizov \& Grigorieva 2006; Ruszkowski, Yang \& Reynolds 2017b) and hot, relativistic plasma-filled 'bubbles' or 'cavities' (perhaps inflated by jets in the first place) around AGN. Different authors have argued that they could help suppress cooling flows by providing additional pressure support to the gas, driving pressurized outflows in the galaxy or CGM, or via heating the CGM/ICM directly via collisional (hadronic \& Coulomb) and streaming-instability losses (Guo \& Oh 2008; Sharma, Parrish \& Quataert 2010; Enßlin et al. 2011; Fujita \& Ohira 2011; Fujita, Kimura \& Ohira 2013; Pfrommer 2013; Wiener, Oh \& Guo 2013; Jacob \& Pfrommer 2017a, b; Pfrommer et al. 2017; Ruszkowski et al. 2017a, b; Jacob et al. 2018).

The direction and geometry of the jet at these scales are also uncertain. The width of the jet can change substantially with time or distance. Although there is still a debate as to whether jets precess or not, several proposed mechanisms including self-induced warping of an irradiated accretion disc (Pringle 1996, 1997), torn accretion discs (Nixon, King \& Price 2012a; Nixon et al. 2012b) due to the LenseThirring effect (Lense \& Thirring 1918), massive black hole binaries (Begelman, Blandford \& Rees 1980), or simply the widely varying angular momentum direction of gas accreting from larger scales on short time-scales (<0.1 Myr; Hopkins et al. 2012; Angles-Alcazar et al. 2020) can plausibly alter the angular momentum direction of the accretion disc, causing jet precession. Multiple observations also suggest jet precession occurs (e.g. Dunn, Fabian \& Sanders 2006; Martí-Vidal et al. 2011; Babul, Sharma \& Reynolds 2013; Aalto et al. 2016). Reflecting such uncertainties, AGN feedback models have adopted energy injection with opening-angles ranging from small or negligible (e.g. Li \& Bryan 2014a, b; Weinberger et al. 2017b; Martizzi et al. 2019) to much wider opening-angles (e.g. Prasad et al. 2015; Hillel \& Soker 2017, 2018) to isotropic (e.g. Reynolds, Balbus \& Schekochihin 2015; Su et al. 2020; Torrey et al. 2020). Several models also consider jet precession (e.g. Li \& Bryan 2014a, b; Yang \& Reynolds 2016a; Bourne \& Sijacki 2017; Martizzi et al. 2019).

In particular, jet precession might more efficiently drive turbulence in the CGM/ICM through changing bulk motion or secondary instabilities (e.g. Li \& Bryan 2014a; Yang \& Reynolds 2016a; ZuHone, Markevitch \& Zhuravleva 2016; Bourne \& Sijacki 2017; Hitomi Collaboration 2018; Martizzi et al. 2019). The enhanced turbulence can also suppress cooling flows by providing direct pressure support to the gas (Parrish et al. 2012), or by heating the gas 'directly' via viscous dissipation (Banerjee \& Sharma 2014; Zhuravleva et al. 2014), effectively conducting heat from the outer hot halo to the inner cool core (Banerjee \& Sharma 2014), or mixing cold structures back into the hot gas in a thermally unstable medium and thereby efficiently re-distributing heat (e.g. Kim \& Narayan 2003; Voigt \& Fabian 2004; Vernaleo \& Reynolds 2006; Parrish, Quataert \& Sharma 2010; Ruszkowski \& Oh 2010; Banerjee \& Sharma 2014). In Paper

${ }^{1}$ Even if magnetic fields are dynamically important on large scales, they can still be critical on scales near the black hole that we do not resolve.
II, we showed that an explicit externally driven turbulence could very effectively quench the galaxy. Here we further test whether a precessing jet can drive such turbulence and thereby quench the galaxy.

Finally, AGN feedback is generally episodic. A period of strong feedback can shut down the cooling flow and the black hole accretion, which subsequently turns off feedback. During the time without feedback, the cooling flows and accretion can be reestablished, starting another episode of feedback, as may have occurred in the Phoenix cluster (Stern et al. 2019). However, the duty cycle and the duration of each episode are highly dependent on both the accretion and feedback models. Most of the literature above only studied a limited part of this large parameter space. In order to narrow down the parameter space of jet launching, in this study we conduct the most extensive set of simulations to date surveying AGN jet launching parameters including energy form (kinetic, thermal, and CR energy), energy flux, mass flux, magnetic field strength and geometry, jet precession angle and period, jet opening-angle, and jet duty cycles. We will test for what part of the parameter space jets can quench galaxies without violating observational constraints on the CGM density and entropy profiles. For viable models, we will also study how and why those models work and what is the required energy.

All of these questions have been studied to a varying extent in the literature already (see references above). However, this work expands on these previous studies in at least four important ways. (i) We attempt a broader and more comprehensive survey, across a wide variety of parameters characterizing jet injection, using an otherwise identical set of physics and numerics, to enable fair comparisons. (ii) We implement all of these in global simulations that self-consistently (and simultaneously) treat the entire halo and star-forming galactic disc. We also reach higher resolution than most previous work, which allows us to resolve more detailed sub-structure in the CGM and galactic disc. (iii) We include explicit, detailed treatments of radiative cooling, the multiphase interstellar medium (ISM) and CGM, star formation, and stellar feedback following the FIRE-2 ${ }^{2}$ simulation physics (Hopkins et al. 2014, 2018b), in order to more robustly model both the gas dynamics and the response of galactic SFRs to cooling flows. (iv) We test our jet model in simulations with magnetohydrodynamics (MHD), conduction, viscosity, and explicit CR transport and dynamics (from AGN) to capture any interaction between the jet and fluid microphysics.

In Section 2, we summarize our initial conditions (ICs) and the AGN jet parameters we survey and describe our numerical simulations. We present our results and describe the observational properties of the more successful runs in Section 3. We discuss the effects of each model in turn, and explain why it works or does not, in Section 4. We discuss the comparison to previous studies and some limitations of our work in Section 5. We summarize in Section 6. We include some observational properties of all the successful and unsuccessful runs in Appendix A.

\section{METHODOLOGY}

We perform simulations of isolated galaxies with a halo mass of $\sim 10^{14} \mathrm{M}_{\odot}$. We set up the initial conditions according to the observed profiles of CC clusters at low redshift, as detailed in Section 2.1. Without any AGN feedback, although the galaxies have initial properties consistent with observations, their cooling flow rates, and SFRs quickly run away, exceeding the observational values by orders of

${ }^{2}$ FIRE project website: http://fire.northwestern.edu 
Table 1. Properties of initial conditions for the simulations/halos studied in this paper.

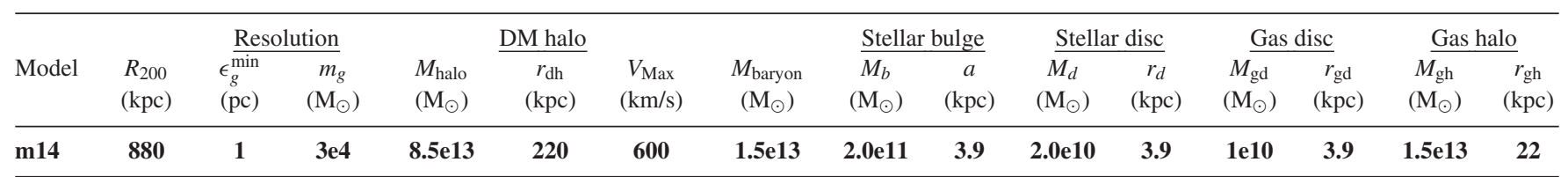

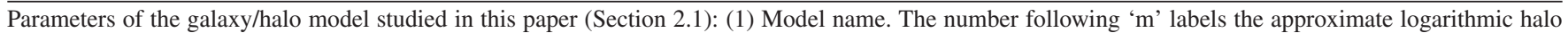

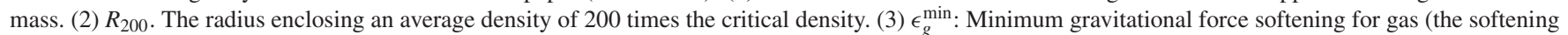

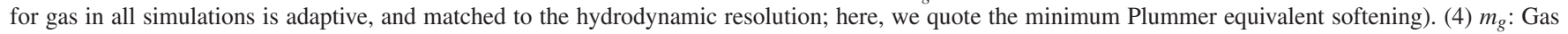

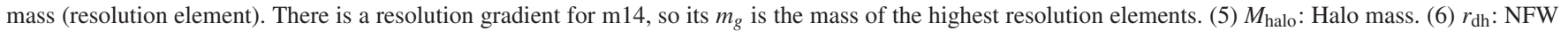

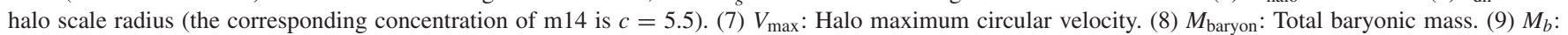

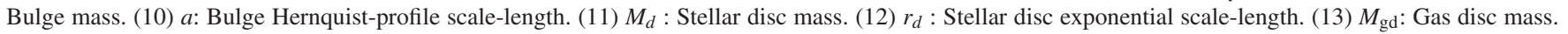
(14) $r_{\mathrm{gd}}$ : Gas disc exponential scale-length. (15) $M_{\mathrm{gh}}$ : Hydrostatic gas halo mass. (16) $r_{\text {gh }}$ : Hydrostatic gas halo $\beta=1 / 2$ profile scale-length.

magnitude (Paper I and Paper II). We evolve the simulations with various AGN jet models and test to what extent (if any) they suppress the cooling flow and whether they can maintain stably quenched galaxies.

We note that while there are more constraints from X-ray observations for rich clusters of mass $\sim 10^{15} \mathrm{M}_{\odot}$, we focus on a galaxy with a halo mass of $10^{14} \mathrm{M}_{\odot}$. The reason is that a halo of this mass already has most of the cooling flow properties of the more massive clusters, and will experience a major cooling catastrophe unless properly quenched, but requires less computational expense. We will consider how jet models scale with halo mass in future work.

Our simulations use GIZMO ${ }^{3}$ (Hopkins 2015), in its meshless finite mass mode, which is a Lagrangian mesh-free Godunov method, capturing advantages of grid-based and smoothed-particle hydrodynamics methods. Numerical implementation details and extensive tests are presented in a series of methods papers for, e.g. the hydrodynamics and self-gravity (Hopkins 2015), MHD (Hopkins 2016; Hopkins \& Raives 2016), anisotropic conduction and viscosity (Hopkins 2017; Su et al. 2017), and CRs (Chan et al. 2019).

All of our simulations have the FIRE-2 implementation of the Feedback In Realistic Environments (FIRE) physical treatments of the ISM, star formation, and stellar feedback, the details of which are given in Hopkins et al. (2018a, b) along with extensive numerical tests. Cooling is followed from 10 to $10^{10} \mathrm{~K}$, including the effects of photo-electric and photo-ionization heating, collisional, Compton, fine structure, recombination, atomic, and molecular cooling.

Star formation is treated via a sink particle method, allowed only in molecular, self-shielding, locally self-gravitating gas, above a density $n>100 \mathrm{~cm}^{-3}$ (Hopkins, Narayanan \& Murray 2013). Star particles, once formed, are treated as a single stellar population with metallicity inherited from their parent gas particle at formation. All feedback rates (SNe and mass-loss rates, spectra, etc.) and strengths are IMF-averaged values calculated from STARBURST99 (Leitherer et al. 1999) with a Kroupa (2002) IMF. The stellar feedback model includes: (1) Radiative feedback including photo-ionization and photo-electric heating, as well as single and multiple-scattering radiation pressure tracked in five bands (ionizing, far-ultraviolet, near-ultraviolet, optical/near-infrared, infrared), (2) original way and AGB winds, resulting in continuous stellar mass loss and injection of mass, metals, energy, and momentum, (3) Type II and Ia $\mathrm{SNe}$ (including both prompt and delayed populations) occurring according to tabulated rates, and injecting the appropriate mass, metals, momentum, and energy to the surrounding gas. All the simulations except the 'B0' run also include MHD, and fully anisotropic conduction, and viscosity with the Spitzer-Braginski coefficients.

${ }^{3} \mathrm{~A}$ public version of this code is available at http://www.tapir.caltech.edu/ $\sim \mathrm{p}$ hopkins/Site/GIZMO.html

\subsection{Initial conditions}

The initial conditions studied here are presented and described in detail in Paper I. The ICs are designed to be similar to observed $\mathrm{CC}$ systems of similar mass wherever possible at $z \sim 0$ (see e.g. Humphrey et al. 2012; Humphrey \& Buote 2013; Su, White \& Miller 2013; Su et al. 2015; Mernier et al. 2017). Their properties are summarized in Table 1 . In this paper, we focus on the $\mathbf{m 1 4}$ halo from Paper I, which has the most dramatic (massive) cooling flow. The dark matter (DM) halo, bulge, black hole, and gas+stellar disc are initialized following Springel \& White (1999) and Springel (2000). We assume a spherical, isotropic, Navarro, Frenk \& White (1996) profile DM halo; a Hernquist (1990) profile stellar bulge $\left(2 \times 10^{12} \mathrm{M}_{\odot}\right)$; an exponential, rotation-supported disc of gas and stars $\left(10^{10}\right.$ and $2 \times 10^{10} \mathrm{M}_{\odot}$, respectively) initialized with Toomre $Q \approx 1$; a $\mathrm{BH}$ with mass $1 / 300$ of the bulge mass (e.g. Häring \& Rix 2004); and an extended spherical, hydrostatic gas halo with a $\beta$-profile $(\beta=1 / 2)$ and rotation at twice the net DM spin (so $\sim 10-15$ per cent of the support against gravity comes from rotation, and most of the support from thermal pressure as expected in a massive halo). All the components of the initial conditions are 'live'. The initial metallicity of the CGM/ICM drops from solar $(Z$ $=0.02)$ to $Z=0.001$ with radius as $Z(r)=0.02(0.05+0.95 /(1+$ $\left.\left.(r / 20 \mathrm{kpc})^{1.5}\right)\right)$. Initial magnetic fields are azimuthal with a seed value of $|\mathbf{B}|=0.3 \mu \mathrm{G} /\left(1+(r / 20 \mathrm{kpc})^{0.375}\right)$ (which will later be amplified) extending throughout the ICM, and the initial CR energy density is in equipartition with the local initial magnetic energy density. The ICs are run adiabatically (no cooling or star formation) to relax any initial transients.

Our $\mathbf{m 1 4}$ halo has an initial cooling rate of $\sim 8 \times 10^{43} \mathrm{erg} \mathrm{s}^{-1}$, with $\sim 3 \times 10^{43} \mathrm{erg} \mathrm{s}^{-1}$ radiated in the X-ray band (0.5-7 kev).

A resolution study is included in the appendix of Paper I. To achieve better convergence, we use a hierarchical super-Lagrangian refinement scheme (Paper I and Paper II) to reach $\sim 3 \times 10^{4} \mathrm{M}_{\odot}$ mass resolution in the core region and around the $\mathrm{z}$-axis where the jet is launched, much higher than many previous global studies. The mass resolution decreases as a function of both radius $\left(r_{3 \mathrm{~d}}\right)$ and distance from the $\mathrm{z}$-axis $\left(r_{2 \mathrm{~d}}\right)$, roughly proportional to $r_{3 \mathrm{~d}}$ and $2^{r_{2 \mathrm{~d}} / 10 \mathrm{kpc}}$, whichever is smaller, down to $2 \times 10^{6} \mathrm{M}_{\odot}$. The highest resolution region is where either $r_{3 \mathrm{~d}}$ or $r_{2 \mathrm{~d}}$ is smaller than $10 \mathrm{kpc}$.

\subsection{AGN Jet Models}

In this paper, we focus on the effects of a given AGN jet. All the jet models are run with a preset mass, energy, and momentum flux: we do not attempt to simultaneously model $\mathrm{BH}$ accretion from $\sim 10-100 \mathrm{pc}$ to the event horizon. We systematically vary the jet 
Table 2. Physics variations (run at highest resolution) in our halo-m14 survey.

\begin{tabular}{|c|c|c|c|c|c|c|c|c|c|c|c|c|c|c|c|c|}
\hline \multirow[b]{2}{*}{ Model } & \multirow[b]{2}{*}{$\begin{array}{l}\Delta T \\
\mathrm{Gyr}\end{array}$} & \multicolumn{2}{|c|}{ Results } & \multicolumn{7}{|c|}{ Input jet fluxes } & \multicolumn{6}{|c|}{ Other jet parameters } \\
\hline & & $\begin{array}{c}\text { SFR } \\
\mathrm{M}_{\odot} \mathrm{yr}^{-1}\end{array}$ & Summary & \multicolumn{4}{|c|}{$\operatorname{erg~s}^{-1}$} & $\begin{array}{c}\dot{M} \\
\mathrm{M}_{\odot} \mathrm{yr}^{-1}\end{array}$ & $\begin{array}{c}\mathrm{v} \\
\mathrm{km} \mathrm{s}^{-1}\end{array}$ & $\begin{array}{c}\dot{P} \\
\text { cgs }\end{array}$ & $\begin{array}{l}\mathrm{T} \\
\mathrm{K}\end{array}$ & $\begin{array}{l}\text { B } \\
\text { G }\end{array}$ & \multicolumn{2}{|c|}{ deg } & \multicolumn{2}{|l|}{ Myr } \\
\hline NoJet & 1.5 & 65 & Strong CF & \multicolumn{5}{|c|}{ N/A } & N/A & & \multicolumn{2}{|c|}{ N/A } & \multicolumn{2}{|c|}{ N/A } & \multicolumn{2}{|l|}{ N/A } \\
\hline \multicolumn{17}{|l|}{ Kinetic energy flux } \\
\hline Kin6e42 & 1.0 & 44 & Strong CF & $5.8 \mathrm{e} 42$ & $1.9 \mathrm{e} 41$ & $8 \mathrm{e} 37-8 \mathrm{e} 41$ & 0 & 2.0 & $3 \mathrm{e} 3$ & $3.9 \mathrm{e} 34$ & $1 \mathrm{e} 7$ & $1 e-3(t)$ & 1 & N/A & N/A & N/A \\
\hline Kin6e 43 & 1.5 & 16 & Slight $\downarrow$ & $5.8 \mathrm{e} 43$ & $1.9 \mathrm{e} 41$ & $1 \mathrm{e} 43$ & 0 & 2.0 & $9.5 \mathrm{e} 3$ & $3.9 \mathrm{e} 34$ & $1 \mathrm{e} 7$ & $1 e-3(t)$ & 1 & N/A & N/A & N/A \\
\hline Kin6e44 & 0.8 & 0 & Overheated & $5.8 \mathrm{e} 44$ & $1.9 \mathrm{e} 41$ & $2 \mathrm{e} 44-3 \mathrm{e} 44$ & 0 & 2.0 & $3 \mathrm{e} 4$ & $3.9 \mathrm{e} 34$ & $1 \mathrm{e} 7$ & $1 e-3(t)$ & 1 & N/A & N/A & N/A \\
\hline \multicolumn{17}{|l|}{ Thermal energy flux } \\
\hline Th6e42 & 1.0 & 27 & Strong CF & $5.8 \mathrm{e} 42$ & $5.8 \mathrm{e} 42$ & $1 \mathrm{e} 39-8 \mathrm{e} 41$ & 0 & 2.0 & $3 \mathrm{e} 3$ & $3.9 \mathrm{e} 34$ & $3 \mathrm{e} 8$ & $1 e-3(t)$ & 1 & N/A & N/A & N/A \\
\hline Th6e43 & 1.5 & 0.51 & Quenched & $5.8 \mathrm{e} 42$ & $5.8 \mathrm{e} 43$ & 2e42 & $\mathbf{0}$ & 2.0 & $3 \mathrm{e} 3$ & $3.9 \mathrm{e} 34$ & $3 e 9$ & 1e-3 (t) & 1 & N/A & N/A & N/A \\
\hline Th6e 44 & 1.0 & 0 & Overheated & $5.8 \mathrm{e} 42$ & $5.8 \mathrm{e} 44$ & $2 \mathrm{e} 43$ & 0 & 2.0 & $3 \mathrm{e} 3$ & $3.9 \mathrm{e} 34$ & $3 \mathrm{e} 10$ & $1 e-3(t)$ & 1 & N/A & N/A & N/A \\
\hline \multicolumn{17}{|l|}{ CR energy flux } \\
\hline CR6e42 & 1.5 & 4.9 & Strong $\downarrow$ & $5.8 \mathrm{e} 42$ & $1.9 \mathrm{e} 41$ & $6 e 41-7 e 41$ & $5.8 \mathrm{e} 42$ & 2.0 & $3 \mathrm{e} 3$ & $3.9 \mathrm{e} 34$ & $1 \mathrm{e} 7$ & $1 e-3(t)$ & 1 & N/A & N/A & N/A \\
\hline CR6e43 & 1.5 & 0.25 & Quenched & $5.8 \mathrm{e} 42$ & $1.9 \mathrm{e} 41$ & $1 \mathrm{e} 42$ & $5.8 \mathrm{e} 43$ & 2.0 & $3 \mathrm{e} 3$ & $3.9 \mathrm{e} 34$ & $1 \mathrm{e} 7$ & $1 e-3(t)$ & 1 & N/A & N/A & N/A \\
\hline CR6e44-B4 & 1.0 & 0 & Overheated & $5.8 \mathrm{e} 42$ & $1.9 \mathrm{e} 41$ & $1 \mathrm{e} 40$ & $5.8 \mathrm{e} 44$ & 2.0 & $3 \mathrm{e} 3$ & $3.9 \mathrm{e} 34$ & $1 \mathrm{e} 7$ & $1 e-3(t)$ & 1 & N/A & N/A & N/A \\
\hline \multicolumn{17}{|l|}{ Magnetic fields } \\
\hline B0 & 1.0 & 64 & Strong CF & $5.8 \mathrm{e} 42$ & $1.9 \mathrm{e} 41$ & 0 & 0 & 2.0 & $3 \mathrm{e} 3$ & $3.9 \mathrm{e} 34$ & $1 \mathrm{e} 7$ & 0 & 1 & N/A & N/A & N/A \\
\hline $\mathrm{B}_{\text {tor }} 1 \mathrm{e}-4$ & 1.0 & 54 & Strong CF & $5.8 \mathrm{e} 42$ & $1.9 \mathrm{e} 41$ & $2 \mathrm{e} 36-1 \mathrm{e} 40$ & 0 & 2.0 & $3 \mathrm{e} 3$ & $3.9 \mathrm{e} 34$ & $1 \mathrm{e} 7$ & $1 e-4(t)$ & 1 & N/A & N/A & N/A \\
\hline $\mathrm{B}_{\text {tor }} 1 \mathrm{e}-3($ Kin6e 42$)$ & 1.0 & 44 & Strong CF & $5.8 \mathrm{e} 42$ & $1.9 \mathrm{e} 41$ & $8 \mathrm{e} 37-8 \mathrm{e} 41$ & 0 & 2.0 & $3 \mathrm{e} 3$ & $3.9 \mathrm{e} 34$ & $1 \mathrm{e} 7$ & $1 e-3(t)$ & 1 & N/A & N/A & N/A \\
\hline $\mathrm{B}_{\text {tor }} 3 \mathrm{e}-3$ & 1.5 & 23 & Strong CF & $5.8 \mathrm{e} 42$ & $1.9 \mathrm{e} 41$ & $2 \mathrm{e} 43$ & 0 & 2.0 & $3 \mathrm{e} 3$ & $3.9 \mathrm{e} 34$ & $1 \mathrm{e} 7$ & $3 e-3(t)$ & 1 & N/A & N/A & N/A \\
\hline $\mathrm{B}_{\mathrm{pol}} 1 \mathrm{e}-4$ & 1.0 & 65 & Strong CF & $5.8 \mathrm{e} 42$ & $1.9 \mathrm{e} 41$ & $3 e 36-5 e 39$ & 0 & 2.0 & $3 \mathrm{e} 3$ & $3.9 \mathrm{e} 34$ & $1 \mathrm{e} 7$ & $1 e-4(p)$ & 1 & N/A & N/A & N/A \\
\hline $\mathrm{B}_{\mathrm{pol}} 1 \mathrm{e}-3$ & 1.0 & 43 & Strong CF & $5.8 \mathrm{e} 42$ & $1.9 \mathrm{e} 41$ & $2 \mathrm{e} 38-4 \mathrm{e} 41$ & 0 & 2.0 & $3 \mathrm{e} 3$ & $3.9 \mathrm{e} 34$ & $1 \mathrm{e} 7$ & $1 e-3(p)$ & 1 & N/A & N/A & N/A \\
\hline $\mathrm{B}_{\mathrm{pol}} 3 \mathrm{e}-3$ & 1.0 & 35 & Strong CF & $5.8 \mathrm{e} 42$ & $1.9 \mathrm{e} 41$ & $2 \mathrm{e} 42-3 \mathrm{e} 42$ & 0 & 2.0 & $3 \mathrm{e} 3$ & $3.9 \mathrm{e} 34$ & $1 \mathrm{e} 7$ & $3 e-3(p)$ & 1 & N/A & N/A & N/A \\
\hline Jet width & & & & & & & & & & & & & & & & \\
\hline Kin6e43-w15 & 1.5 & 19 & Slight $\downarrow$ & $5.8 \mathrm{e} 43$ & $1.9 \mathrm{e} 41$ & $1 \mathrm{e} 43-2 \mathrm{e} 43$ & 0 & 2.0 & $9.5 \mathrm{e} 3$ & $3.9 \mathrm{e} 34$ & $1 \mathrm{e} 7$ & $1 e-3(t)$ & 15 & N/A & N/A & N/A \\
\hline Kin6e43-w30 & 1.5 & 11 & Slight $\downarrow$ & $5.8 \mathrm{e} 43$ & $1.9 \mathrm{e} 41$ & $1 \mathrm{e} 43-2 \mathrm{e} 43$ & 0 & 2.0 & $9.5 \mathrm{e} 3$ & $3.9 \mathrm{e} 34$ & $1 \mathrm{e} 7$ & $1 \mathrm{e}-3(\mathrm{t})$ & 30 & N/A & N/A & N/A \\
\hline Kin6e43-w45 & 1.5 & 7.1 & Slight $\downarrow$ & $5.8 \mathrm{e} 43$ & $1.9 \mathrm{e} 41$ & $1 \mathrm{e} 43-2 \mathrm{e} 43$ & 0 & 2.0 & $9.5 \mathrm{e} 3$ & $3.9 \mathrm{e} 34$ & $1 \mathrm{e} 7$ & $1 e-3(t)$ & 45 & N/A & N/A & N/A \\
\hline Kin6e43-wiso & 1.5 & 0 & Quenched & $5.8 \mathrm{e} 43$ & $1.9 \mathrm{e} 41$ & $2 \mathrm{e} 43$ & $\mathbf{0}$ & 2.0 & $9.5 \mathrm{e} 3$ & $3.9 \mathrm{e} 34$ & $1 \mathrm{e} 7$ & $1 e-3(t)$ & iso & N/A & N/A & N/A \\
\hline Jet precession & & & & & & & & & & & & & & & & \\
\hline Kin6e43-pr15- $t_{p} 10$ & 1.5 & 17 & Slight $\downarrow$ & $5.8 \mathrm{e} 43$ & $1.9 \mathrm{e} 41$ & $1 \mathrm{e} 43$ & 0 & 2.0 & $9.5 \mathrm{e} 3$ & $3.9 \mathrm{e} 34$ & $1 \mathrm{e} 7$ & $1 e-3(t)$ & 1 & 15 & N/A & 10 \\
\hline Kin6e43-pr30- $\mathrm{t}_{p} 10$ & 1.5 & 12 & Slight $\downarrow$ & $5.8 \mathrm{e} 43$ & $1.9 \mathrm{e} 41$ & $1 \mathrm{e} 43$ & 0 & 2.0 & $9.5 \mathrm{e} 3$ & $3.9 \mathrm{e} 34$ & $1 \mathrm{e} 7$ & $1 \mathrm{e}-3(\mathrm{t})$ & 1 & 30 & N/A & 10 \\
\hline Kin6e43-pr45-t ${ }_{p} 10$ & 1.5 & 1.5 & Strong $\downarrow$ & $5.8 \mathrm{e} 43$ & $1.9 \mathrm{e} 41$ & $1 \mathrm{e} 43$ & $\mathbf{0}$ & 2.0 & $9.5 \mathrm{e} 3$ & $3.9 \mathrm{e} 34$ & $1 \mathrm{e} 7$ & $1 e-3(t)$ & 1 & 45 & N/A & 10 \\
\hline Kin6e43-pr30-t $t_{p} 100$ & 1.5 & 3.0 & Strong $\downarrow$ & $5.8 \mathrm{e} 43$ & $1.9 \mathrm{e} 41$ & $1 \mathrm{e} 43$ & $\mathbf{0}$ & 2.0 & $9.5 \mathrm{e} 3$ & $3.9 \mathrm{e} 34$ & $1 \mathrm{e} 7$ & $1 e-3(t)$ & 1 & 30 & N/A & 100 \\
\hline Kin6e43-pr45-t 100 & 1.5 & 1.0 & Quenched & $5.8 \mathrm{e} 43$ & $1.9 \mathrm{e} 41$ & $1 e 43-3 e 43$ & $\mathbf{0}$ & 2.0 & $9.5 \mathrm{e} 3$ & $3.9 \mathrm{e} 34$ & $1 \mathrm{e} 7$ & $1 e-3(t)$ & 1 & 45 & N/A & 100 \\
\hline Kinetic specific ener & & & & & & & & & & & & & & & & \\
\hline Kin6e42-B4-m2e-2 & 1.0 & 43 & Strong CF & $5.8 \mathrm{e} 42$ & $1.9 \mathrm{e} 39$ & $4 \mathrm{e} 34-1 \mathrm{e} 41$ & 0 & 0.02 & $3 \mathrm{e} 4$ & $3.9 \mathrm{e} 32$ & $1 \mathrm{e} 7$ & $1 e-4(t)$ & 1 & N/A & N/A & N/A \\
\hline Kin6e43-B4-m2e-1 & 1.5 & 17 & Slight $\downarrow$ & $5.8 \mathrm{e} 43$ & $1.9 \mathrm{e} 40$ & $9 \mathrm{e} 40-4 \mathrm{e} 42$ & 0 & 0.2 & $3 e 4$ & $3.9 \mathrm{e} 33$ & $1 \mathrm{e} 7$ & $1 e-4(t)$ & 1 & N/A & N/A & N/A \\
\hline Kin6e44-B4 & 1.5 & 13 & Slight $\downarrow$ & $5.8 \mathrm{e} 44$ & $1.9 \mathrm{e} 41$ & $3 e 42-5 e 42$ & 0 & 2.0 & $3 e 4$ & $3.9 \mathrm{e} 34$ & $1 \mathrm{e} 7$ & 1e-4 (t) & 1 & N/A & N/A & N/A \\
\hline Thermal specific ene & & & & & & & & & & & & & & & & \\
\hline Th6e42-B4-m2e-2 & 1.5 & 29 & Slight $\downarrow$ & $5.8 \mathrm{e} 40$ & $5.8 \mathrm{e} 42$ & $3 e 39-2 \mathrm{e} 41$ & 0 & 0.02 & $3 \mathrm{e} 3$ & $3.9 \mathrm{e} 32$ & $3 \mathrm{e} 10$ & $1 e-4(t)$ & 1 & N/A & N/A & N/A \\
\hline Th6e42-B4-m2e-1 & 1.0 & 29 & Strong CF & $5.8 \mathrm{e} 42$ & $1.9 \mathrm{e} 40$ & $5 e 40-1 e 42$ & 0 & 0.2 & $3 \mathrm{e} 3$ & $3.9 \mathrm{e} 33$ & $3 \mathrm{e} 9$ & $1 e-4(t)$ & 1 & N/A & N/A & N/A \\
\hline Th6e43-B4-m2e-1 & 1.0 & 0 & Overheated & $5.8 \mathrm{e} 41$ & $5.8 \mathrm{e} 43$ & $2 \mathrm{e} 42-9 \mathrm{e} 42$ & 0 & 0.2 & $3 \mathrm{e} 3$ & $3.9 \mathrm{e} 33$ & $3 \mathrm{e} 10$ & $1 e-4(t)$ & 1 & N/A & N/A & N/A \\
\hline Th6e43-B4 & 1.5 & 4.3 & Strong $\downarrow$ & $5.8 \mathrm{e} 43$ & $1.9 \mathrm{e} 41$ & $2 \mathrm{e} 40$ & $\mathbf{0}$ & 2.0 & $3 \mathrm{e} 3$ & $3.9 \mathrm{e} 34$ & $3 \mathrm{e} 9$ & $1 e-4(t)$ & 1 & N/A & N/A & N/A \\
\hline Th6e44-B4 & 1.2 & 0 & Overheated & $5.8 \mathrm{e} 42$ & $5.8 \mathrm{e} 44$ & $2 \mathrm{e} 41$ & 0 & 2.0 & $3 \mathrm{e} 3$ & $3.9 \mathrm{e} 34$ & $3 \mathrm{e} 10$ & $1 e-4(t)$ & 1 & N/A & N/A & N/A \\
\hline CR specific energy & & & & & & & & & & & & & & & & \\
\hline CR6e43-B4-m2e-1 & 1.5 & $\mathbf{0}$ & Quenched & $5.8 \mathrm{e} 41$ & $1.9 \mathrm{e} 40$ & $1 \mathrm{e} 42-6 \mathrm{e} 42$ & $5.8 \mathrm{e} 43$ & 0.2 & $3 e 3$ & $3.9 \mathrm{e} 33$ & $1 \mathrm{e} 7$ & $1 e-4(t)$ & 1 & N/A & N/A & N/A \\
\hline CR6e43-B4 & 1.5 & 0.12 & Quenched & $5.8 \mathrm{e} 42$ & $1.9 \mathrm{e} 41$ & $1 \mathrm{e} 40$ & $5.8 \mathrm{e} 43$ & 2.0 & $3 \mathrm{e} 3$ & $3.9 \mathrm{e} 34$ & $1 \mathrm{e} 7$ & $1 e-4(t)$ & 1 & N/A & N/A & N/A \\
\hline Duty cycle & & & & & & & & & & & & & & & & \\
\hline Th6e44-B4-t ${ }_{d} 10$ & 0.6 & 0 & Overheated & $5.8 \mathrm{e} 42$ & $5.8 \mathrm{e} 44$ & $8 \mathrm{e} 41-2 \mathrm{e} 43$ & 0 & 2.0 & $3 \mathrm{e} 3$ & $3.9 \mathrm{e} 34$ & $3 \mathrm{e} 10$ & $1 \mathrm{e}-4(\mathrm{t})$ & 1 & N/A & $1 / 10$ & N/A \\
\hline Th6e44-B4-t $_{d} 100$ & 1.5 & 0.064 & Quenched & $5.8 \mathrm{e} 42$ & $5.8 \mathrm{e} 44$ & 2e41-6e41 & $\mathbf{0}$ & 2.0 & $3 \mathrm{e} 3$ & $3.9 \mathrm{e} 34$ & $3 \mathrm{e} 10$ & 1e-4 (t) & 1 & N/A & $10 / 100$ & N/A \\
\hline
\end{tabular}

This is a partial list of simulations studied here: each was run using halo $\mathbf{m 1 4}$, systematically varying the jet parameters. Columns list: (1) Model name: The naming of each model starts with the primary form of energy flux and the energy flux value in $\mathrm{erg} \mathrm{s}^{-1}$ used. A run with 'B4' in the name means the initial jet magnetic field has a toroidal geometry with a maximum field strength

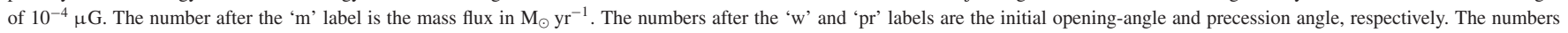
after the ' $t_{p}$ ' and ' $t_{d}$ ' label the precession period and duty cycle period. If a specific quantity is not labeled in the name, the jet model is launched with a constant mass flux of $2 \mathrm{M}_{\odot} \mathrm{yr}^{-1}$, toroidal magnetic field, with a maximum field strength of $10^{-3} \mu \mathrm{G}, 1^{\circ}$ opening-angle, no precession, and $100 \%$ duty cycle. (2) $\Delta T$ : Simulation duration. All simulations are run to 1.5 Gyr, unless either the

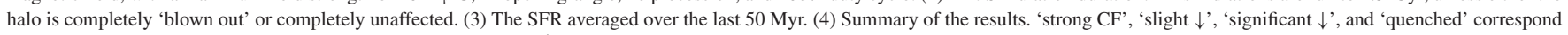
respectively to a SFR of $\gtrsim 20, \sim 5-20, \sim 1-5$ and $\lesssim 1 \mathrm{M}_{\odot} \mathrm{yr}^{-1}$. 'Overheated' means the jet explosively destroys the cooling flow in $<500 \mathrm{Myr}$, leaving a core with much lower density and

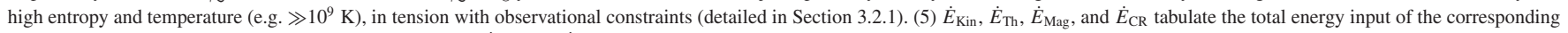

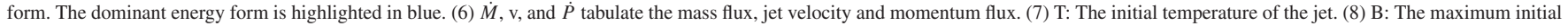

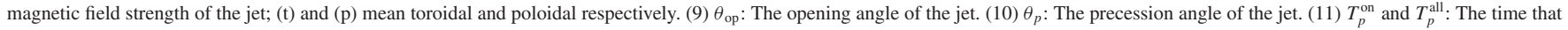
the jet is on in each duty cycle and the period of the duty cycle. (12) $T_{p}$ : Precession period.

velocity, energy composition (kinetic, thermal, magnetic, and $\mathrm{CR}$ energy), mass flux, opening-angle, procession, and duty cycle. We note that such variations reflect the uncertainties from the nature of AGN jets and the sub-resolution $(<10 \mathrm{pc})$ physics around the black hole, which affects the balance of different energy forms and other jet parameters. A full list of simulations can be found in Table 2. We emphasize that the parameters in the table reflect the jet parameters at our launch scale. The jet properties will continuously evolve as it interacts with the surrounding gas.

We launch the jet with a particle spawning method, which creates new gas cells (resolution elements) from the central black hole. With this method, we have better control of the jet properties as 
the launching is less dependent on the neighbour-finding results. We can also enforce a higher resolution for the jet elements. The numerical method in this paper is similar to Torrey et al. (2020), which studied the effects of broad absorption line (BAL) wind feedback on disc galaxies. The spawned gas particles have a mass resolution of $5000 \mathrm{M}_{\odot}$ and are forbidden to de-refine (merge into a regular gas element) before they decelerate to 10 percent of the launch velocity. Two particles are spawned in opposite z-directions at the same time when the accumulated jet mass flux reaches twice the target spawned particle mass, so linear momentum is always exactly conserved. Initially, the spawned particle is randomly placed on a sphere with a radius of $r_{0}$, which is either $10 \mathrm{pc}$ or half the distance between the black hole and the closest gas particle, whichever is smaller. If the particle is initialized at a position $\left(r_{0}, \theta_{0}, \phi_{0}\right)$ and the jet opening-angle of a specific model is $\theta_{\mathrm{op}}$, the initial velocity direction of the jet will be set at $2 \theta_{\mathrm{op}} \theta_{0} / \pi$ for $\theta_{0}<\pi / 2$ and at $\pi-$ $2 \theta_{\text {op }}\left(\pi-\theta_{0}\right) / \pi$ for $\theta_{0}>\pi / 2$. With this, the projected paths of any two particles will not intersect.

The naming of each model starts from the primary form of energy at our injection scale ('Kin', 'Th', and 'CR' for kinetic, thermal, and CR energy, respectively) and the corresponding energy flux in $\mathrm{erg} \mathrm{s}^{-1}$. The run with 'B4' in the name means that the initial jet magnetic field is toroidal with a maximum field strength of $10^{-4} \mathrm{G}$. The number after the ' $m$ ' label provides the mass flux in units of $\mathrm{M}_{\odot} \mathrm{yr}^{-1}$. The number after ' $\mathrm{w}$ ' and 'pr' gives the initial openingangle and precession angle in degrees. The number after ' $t_{p}$ ' and ' $t_{d}$ ' denotes the precession period and the duty cycle in Myr. If a specific quantity is not labeled in the name, the jet model has fiducial values of a constant mass flux of $2 \mathrm{M}_{\odot} \mathrm{yr}^{-1}$, an initial toroidal magnetic field with a maximum field strength of $10^{-3}, 1^{\circ}$ opening-angle, no precession, and 100 per cent duty cycle (i.e. we only label runs with parameters that differ from these default values).

\subsubsection{Form of jet energy}

Each spawned jet element carries mass, velocity, thermal energy, magnetic field, and CR energy, so the energy flux of each kind is well controlled. In the initial conditions, we set the blackhole mass at $10^{9} \mathrm{M}_{\odot}$, corresponding to an Eddington luminosity $L_{\text {Edd }} \sim 10^{47}$ $\mathrm{erg} \mathrm{s}^{-1}$. We systemically test the jet model with energy input in each form ranging from $\sim 6 \times 10^{42}$ up to $6 \times 10^{44} \mathrm{erg} \mathrm{s}^{-1}$, which corresponds to roughly $6 \times 10^{-5}-6 \times 10^{-3} L_{\mathrm{Edd}}$, around the total $\mathrm{X}$-ray luminosity of the whole system and the plausible energy flux according to Paper II.

Each energy form is briefly described below:

(i) Kinetic component: Despite the relativistic nature of jets at the black hole scale, such scales are orders of magnitude smaller than the finest scale we can resolve. Instead, we have to initiate the spawned element at $\sim 10 \mathrm{pc}$, at which point the jet has already been decelerated significantly. Moreover, we are also constrained by the Newtonian nature of our MHD solver, which cannot accurately treat relativistic velocities. Accordingly, we leave the jet element's initial spawning velocity as a free parameter varied within $3000-30000 \mathrm{~km} \mathrm{~s}^{-1}$. This roughly spans the range from the minimum velocity required to sustain a clear bi-polar jet shape to the maximum feasible velocity, as limited by the numerical methods and computational time.

(ii) Thermal component: At the scale where we initiate the jet, a significant amount of energy has probably already been thermalized, but the fraction is uncertain. Therefore, we also leave the internal temperature of the jet plasma as a free parameter ranging from $10^{7}$ to $3 \times 10^{10} \mathrm{~K}$, corresponding to roughly the same range of specific energy as the kinetic jet models we studied. We emphasize that due to the injection in jet form, this is very different from 'traditional' thermal feedback, which we will discuss later.

(iii) Magnetic component:

Given the uncertainties of the magnetic field strength and geometry at the scale where we launch our jet, we parametrize the initial magnetic fields as either purely toroidal or purely poloidal with different strengths. The toroidal magnetic fields follow

$B_{\mathrm{tor}, \phi} \propto r \sin \theta \exp \left(-\frac{r^{2}}{r_{\mathrm{inj}}^{2}}\right)$,

where $r_{\text {inj }}$ is set to $10 \mathrm{pc}$. The poloidal magnetic fields follow

$B_{\mathrm{pol}, r} \propto \frac{r \sin \theta r \cos \theta}{r_{\mathrm{inj}}^{2}} \exp \left(-\frac{r^{2}}{r_{\mathrm{inj}}^{2}}\right)$,
$B_{\mathrm{pol}, z} \propto\left(1-\frac{r^{2} \sin ^{2} \theta}{r_{\mathrm{inj}}^{2}}\right) \exp \left(-\frac{r^{2}}{r_{\mathrm{inj}}^{2}}\right)$,

(e.g. Guan et al. 2014). ${ }^{4}$

We study models with a maximum initial magnetic field strength in the jet plasma ranging from $10^{-4} \mu \mathrm{G}$ to $3 \times 10^{-3} \mu \mathrm{G}$ (roughly the maximum feasible magnetic field strength limited by the numerical method and computational time). We use a toroidal magnetic field configuration with the maximum field strength at $10^{-3} \mu \mathrm{G}$ as the fiducial parameters since it is roughly the minimum value able to clearly affect the global magnetic field configurations.

(iv) Cosmic rays: We treat this component analogously to our 'thermal jet' runs - simply injecting a fixed specific CR energy with the spawned jet elements, i.e. assuming a constant fraction of the jet plasma energy is in CRs. The CR physics and numerical implementation are described in detail in Chan et al. (2019). Briefly, this treats CRs including streaming (at the local Alfvén speed, with the appropriate streaming loss term, which thermalizes, following Uhlig et al. 2012, but with $v_{\text {st }}=v_{A}$ ), diffusion with a fixed diffusivity $\kappa_{\mathrm{CR}}$, adiabatic energy exchange with the gas and CR pressure, and hadronic and Coulomb losses (following Guo \& Oh 2008). We follow a single energy bin (i.e. $\mathrm{GeV}$ proton $\mathrm{CRs}$, which dominate the pressure), treated in the ultra-relativistic limit. Streaming and diffusion are fully-anisotropic along magnetic field lines. In Chan et al. (2019) and Hopkins et al. (2019, 2021c), we showed that matching observed $\gamma$-ray luminosities in simulations with the physics above requires $\kappa_{\mathrm{CR}} \sim 10^{29} \mathrm{~cm}^{2} \mathrm{~s}^{-1}$, in good agreement with detailed CR transport models that include an extended gaseous halo around the Galaxy (see e.g. Strong \& Moskalenko 1998; Strong et al. 2010; Trotta et al. 2011), so we adopt this as our fiducial value..$^{5,6}$

We study models with CR energy fluxes ranging from $6 \times 10^{42}-$ $6 \times 10^{43} \mathrm{erg} \mathrm{s}^{-1}$, roughly the values suggested to be capable of stably

${ }^{4}$ Given that the spawned particle is launched at a radius of $10 \mathrm{pc}$ (most of the time, since the closest neighbourhood gas cell rarely goes closer to the black hole), we always set the exponential part in the poloidal expression (except for $\mathrm{B}_{\mathrm{pol}} 3 \mathrm{e}-3$ where we used the full expression) and the $r$ times exponential part in the toroidal expression to a constant. This only deviates from the above expressions during the time in a run when there is a very strong cooling flow $\left(\mathrm{SFR} \gtrsim 45 \mathrm{M}_{\odot} \mathrm{yr}^{-1}\right)$. During those times, the closest particle to the black hole is smaller than $10 \mathrm{pc}$ and therefore the jet particles are spawned at a smaller radius. However, magnetic fields do very little in those cases anyway, and none of the conclusions should change. We also checked the $\mathrm{B}_{\mathrm{pol}} 1 \mathrm{e}-3$ case to confirm that such variation does not cause any qualitative difference.

${ }^{5}$ We caution that we do not account for the possibility of different diffusion coefficients in different environments (see e.g. Hopkins et al. 2021b, c).

${ }^{6} \mathrm{We}$ also note that in runs with $\mathrm{CR}$ jets, CRs from $\mathrm{SNe}$ are not included, so we have a clean test of the impact of AGN CR jets. We showed in Paper I that CRs from stars have little effect on the cooling flows in massive galaxies. 
quenching a $10^{14} \mathrm{M}_{\odot}$ halo in our previous study with isotropic energy injection (Paper II).

\subsubsection{Mass flux and specific energy}

All of the tested runs have a constant mass flux, unless we specify a duty cycle below unity. We explicitly test the jet models with the same energy flux in each form but with a different mass flux and therefore specific energy. The tested mass fluxes range from 0.02 to $2 \mathrm{M}_{\odot} \mathrm{yr}^{-1}$ (0.01-1 $L_{\text {Edd }} / c^{2}$ ), roughly comparable to the values obtained in the AGN feedback models considered in the literature (e.g. Yang et al. 2012; Li et al. 2015; Prasad et al. 2015). Correspondingly, the kinetic, thermal, or CR energy per unit mass we tested ranged from $\sim 5 \times 10^{16}$ to $5 \times 10^{18} \mathrm{erg} \mathrm{g}^{-1}$, so that the total energy flux is comparable to the halo cooling rate and the required energy flux suggested in Paper II.

\subsubsection{Jet opening-angle}

Due to our jet spawning method, we are able to strictly control the initial opening-angle of the jet. We emphasize that this is the initial opening-angle, which may change as the jet expands or collimates due to external interactions. We perform most of our runs with a default initial jet opening-angle of $1^{\circ}$; however, we explicitly tested different jet opening-angles ranging from $1^{\circ}$ (a very narrow jet) to completely isotropic (resembling a BAL wind model; e.g. Hopkins et al. 2016; Torrey et al. 2020).

\subsubsection{Jet precession}

We tested jet precession with different precession angles and periods. The angles ranged from $15^{\circ}$ to $45^{\circ}$, and the periods ranged from 10 to $100 \mathrm{Myr}$, a broader range than the values usually quoted in the literature $\left(\sim 8-15^{\circ}, 5-10 \mathrm{Myr}\right.$; e.g. Li \& Bryan 2014b; Yang \& Reynolds 2016a; Bourne \& Sijacki 2017).

\subsubsection{Jet duty cycles and episodic lifetimes}

Cycles of AGN jets are observed and can naturally occur in simulations with a self-consistently coupled AGN accretion and feedback model. Given that we do not intend to model accretion explicitly in this study, we test models with preset fixed duty cycles and episodic life times. In 'Th6e44-B4- $\mathrm{t}_{d} 10$ ' the mass flux follows $\dot{M} \propto \sin ^{2}(\pi t / 2 \mathrm{Myr})$ 'on' for $2 \mathrm{Myr}$ and is then turned off $(\dot{M}=0)$ for $8 \mathrm{Myr}$, before repeating. In 'Th6e44-B4-t ${ }_{d} 100$ ', the mass flux is constant when 'on' at $\dot{M}=2 \mathrm{M}_{\odot} \mathrm{yr}^{-1}$ for $10 \mathrm{Myr}$ and then off for 90 Myr. When the jet is on, both models have a specific thermal energy of $\sim 5 \times 10^{17} \mathrm{erg} \mathrm{g}^{-1}$; the label ' $6 \mathrm{e} 44$ ' in the model name refers to its energy flux at the peak, in $\operatorname{erg~s}^{-1}$. In both runs, the averaged energy flux is $6 \times 10^{43} \mathrm{erg} \mathrm{s}^{-1}$, and the duty cycle (percentage of time that a jet is on) is 10-20 per cent and the recurrence time of the jet $(10-100 \mathrm{Myr})$ is broadly within the observational range (e.g. McNamara \& Nulsen 2007, and references therein) and the range of values seen in self-regulating AGN jet simulations in the literature (e.g. Prasad et al. 2015; Li et al. 2015; Yang \& Reynolds 2016a).

\subsection{Comparing to observational constraints on halo gas}

In this work, we quantify the SFR (Fig. 1), the core baryonic mass (Fig. 2) and gas fraction (Fig. 3), the total X-ray luminosity (Fig. 4), and the gas density, entropy (Fig. 5), and velocity dispersion profiles (Fig. 6) of each run. We constrain the model through a qualitative comparison to the observed X-ray luminosity, gas density and entropy profiles, and turbulent velocity.

For the X-ray luminosity, we compare with results from Reiprich \& Böhringer (2002) and Stanek et al. (2006), who provide the total $\mathrm{X}$-ray luminosities in the $0.1-2.4 \mathrm{keV}$ band as a function of $M_{200}$. In Fig. 4, we calculate the X-ray luminosity in the 0.5-7 kev band using the same methods as in Schure et al. (2009) and Ressler, Quataert \& Stone (2018), in which the cooling curve is calculated for photospheric solar abundances (Lodders 2003) using the spectral analysis code SPEX (Kaastra, Mewe \& Nieuwenhuijzen 1996) and scaled according to the local hydrogen, helium, and metal mass fractions. Given that most of the X-ray emission from halo gas in haloes in this mass range is below $2 \mathrm{keV}$, the comparison here is not particularly sensitive to such variations. We integrate the total emission in our simulations out to a radius of $\sim R_{200}$ and average over the last $50 \mathrm{Myr}$ of each run.

We also compare the density, luminosity-weighted density, and entropy profiles to the scaled observational density profiles for CC and NCC (red) clusters (McDonald et al. 2013) in Figs 5, A1, and A2. For the density profiles, we use the panel for $z<0.1$ from fig. 9 (central left) of McDonald et al. (2013) and scale by the $z=0$ critical density to convert from their overdensity values and use $r_{500}=560 \mathrm{kpc}$ (our initial condition) to scale the radial coordinate. The lightened curves in the bottom row of Fig. 5 and in Fig. A2 indicate the observational entropy profiles for CC (blue) and NCC (red) clusters (McDonald et al. 2013), from their fig. 2. We note that the haloes in McDonald et al. (2013) have a mass range of $\sim 2 \times 10^{14}<M_{500}<20 \times 10^{14} \mathrm{M}_{\odot} / h_{70}$ and therefore to account for the lower virial temperature of our halo, which is smaller by approximately a factor of three from their median cluster, we scale their entropy profile by this same factor. We note that the luminosity-weighted density, temperature, and entropy are not computed in the same way as the observed quantities, so the comparison should be viewed qualitatively; in particular, the disc and jet may give rise to sharp features in the profiles at $r<10-20 \mathrm{kpc}$ which would not be seen with a more careful observational comparison. We describe how we define a galaxy to be overheated or non-overheated given the entropy and density constraints in Section 3.2.1.

We estimate the turbulent velocity upper-bound in $T>10^{7} \mathrm{~K}$ gas in our runs by excluding radial motion to account for the chaotic component in Figs 6. We compare with the observed turbulent velocity in the Perseus cluster $\left(\$ 200 \mathrm{~km} \mathrm{~s}^{-1}\right.$; Hitomi Collaboration 2016, 2018). We emphasize that Perseus is a much more massive halo $\left(5-6 \times 10^{14} \mathrm{M}_{\odot}\right)$ than the ones studies here, and the velocity quoted above is taken only as a reference point.

Finally, we constrain the $\sim \mathrm{GeV}$ gamma-ray luminosity from hadronic interactions in our runs with CRs by the current observational upper limits (Ackermann et al. 2016; Wiener \& Zweibel 2019). Likewise, we estimated the $\sim \mathrm{GHz}$ radio luminosities from secondary $\mathrm{CR}$ electrons from the radio flux, assuming all the secondary CR electrons decay via synchrotron emission, and compare them to (Giacintucci et al. 2014; Bravi, Gitti \& Brunetti 2016). The gammaray energy emission per volume is roughly $\dot{e}_{\gamma} \sim 5 / 6 * \Lambda_{\text {had }} e_{\mathrm{CR}} n$, where $\Lambda_{\text {had }} \sim 7.51 \times 10^{-16} s^{-1}$ is the hadronic coupling coefficient, $e_{\mathrm{CR}}$ is the CR energy density, and $n$ is the number density. The radio flux per volume in $\mathrm{GHz}$ from secondary electrons is roughly $\dot{e}_{\text {radio }} \sim f_{\mathrm{GHz}}(2 / 3)(1 / 4)(5 / 6) \Lambda_{\mathrm{had}} e_{\mathrm{CR}} n$, where $f_{\mathrm{GHz}} \sim 0.01$ is the fraction of energy flux in the $\mathrm{GHz}$ band. To make more detailed predictions for radio emission, explicitly modeling CR electrons will be required; this is left for future work. 

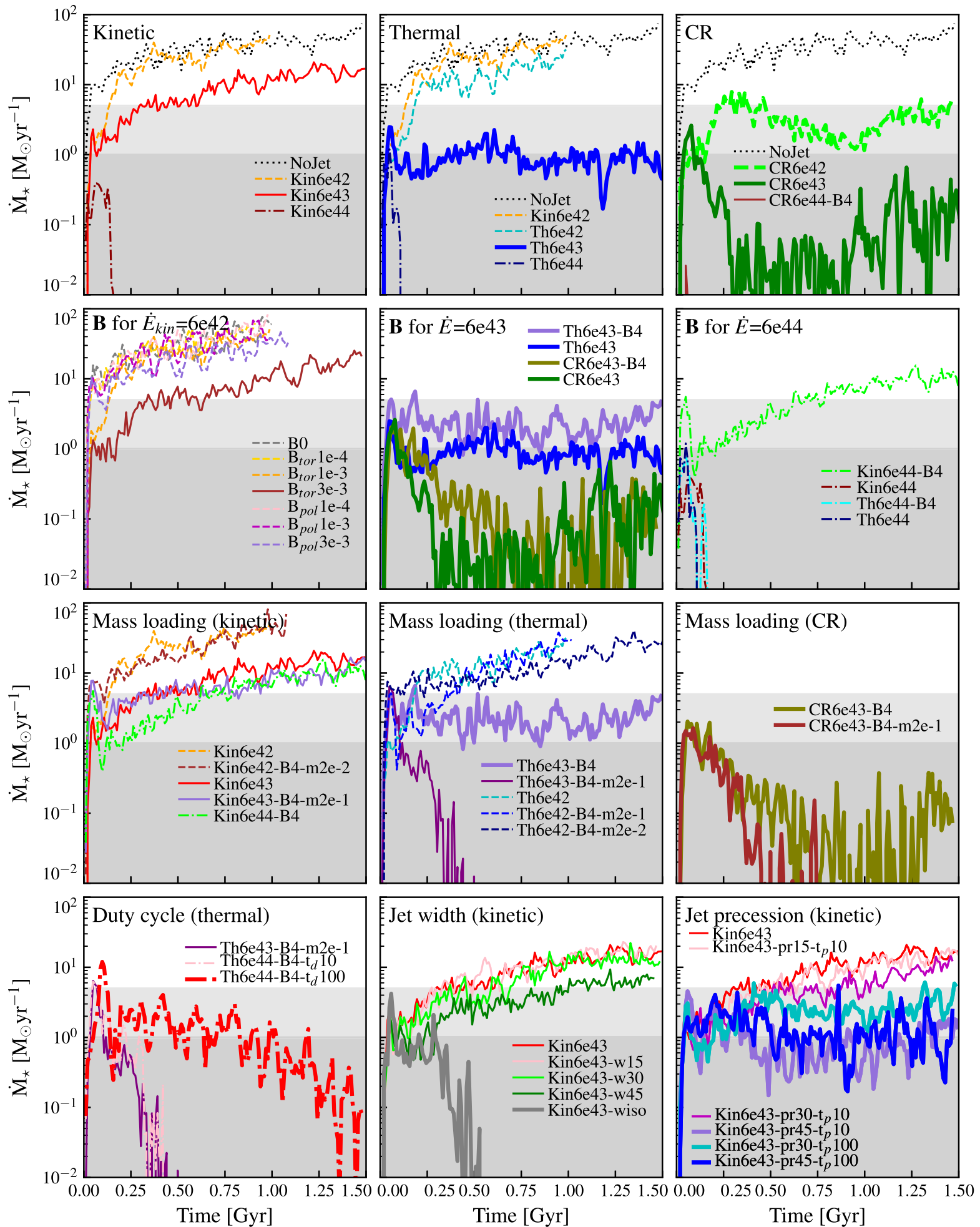

Figure 1. The SFR as a function of time for all runs. Each panel labels the corresponding parameters we vary, and we also show the NoJet (no jet feedback) case in the top three panels. The thick lines indicate the quiescent and 'non-overheated' cases. The grey areas at the bottom indicates an $\mathrm{SFR}_{\text {below }} \sim 5 \mathrm{M}_{\odot} \mathrm{yr}^{-1}$ and (darker grey) $\sim 1 \mathrm{M}_{\odot} \mathrm{yr}^{-1}$, which we defined as 'strong $\downarrow$ ' and 'quenched' respectively in Table 2 . Kinetic jets are the least effective in suppressing the SFR unless the opening-angle or precession angle is set to be wider than $30-45^{\circ}$, or is significantly widened by magnetic fields. Thermal jets can more effectively quench the galaxy when the energy input reaches $\sim 6 \times 10^{43} \mathrm{erg} \mathrm{s}^{-1}$. CR jets quench the most efficiently, requiring an energy input of only $\sim 6 \times 10^{42}$ erg s ${ }^{-1}$ In the sampled parameter space, magnetic fields cause less than a factor $\sim 2$ effect in most cases. For the same thermal or CR input, lower mass flux and the higher specific energy produces more effective quenching. With the same averaged mass flux and thermal energy flux, duty cycles with periods « $100 \mathrm{Myr}$ are effectively the same as 'continuous' jets at these $\gg$ kpc scales, while models with $\sim 10$ per cent duty cycles spread over $\gtrsim 100$ Myr periods are less effective than continuous or short period jets. 

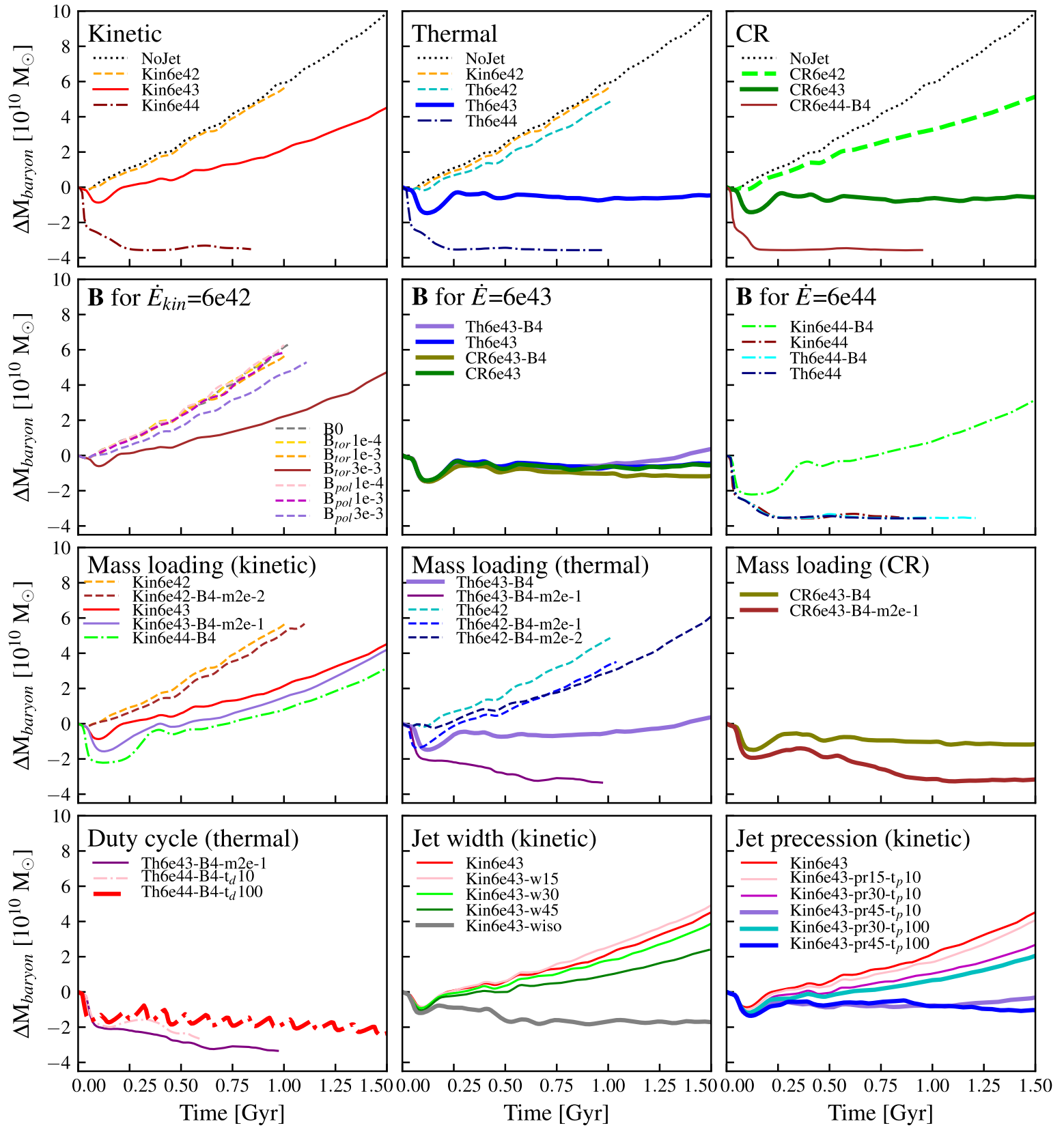

Figure 2. The evolution of the core baryonic mass (total star + gas mass within $30 \mathrm{kpc}$ ) as a function of time. The panels and runs follow the same grid and style as in Fig. 1. Most of the quiescent non-overheated runs (indicated with thick lines) have a core baryonic mass that remains almost constant over the duration of the run, indicating an explicit suppression of the cooling flow. The only exception is the lower flux CR jet run ('CR6e42'), where there is still a non-negligible increase of core baryonic mass even while the SFR is significantly suppressed.

Unless otherwise noted, we define the core radius as $30 \mathrm{kpc}$ $\left(\sim 0.05 R_{500}\right)$. This is comparable to the cooling radius $\left(R_{\text {cool }} \sim\right.$ $30-50 \mathrm{kpc})$, which we define as the radius within which the cooling time is less than our simulation time $(\sim 1-2 \mathrm{Gyr})$.

\section{RESULTS}

In this section, we summarize the results of all our simulations before turning to a more detailed analysis of individual mechanisms. Section 3.1 describes the star formation and cooling-flow properties of all the runs. In Section 3.2, we further show the key X-ray observational properties of the quenched runs labeled as 'strong $\downarrow$ ' (SFR $\sim 1-5 \mathrm{M}_{\odot} \mathrm{yr}^{-1}$ ) or 'quenched' ( $\mathrm{SFR}<1 \mathrm{M}_{\odot} \mathrm{yr}^{-1}$ ) in Table 2, which do not 'overheat' the halo (high entropy and low density within the cooling radius qualitatively inconsistent with $\mathrm{X}$-ray observations).

\subsection{Star formation history and baryonic inflow}

Figs 1, 2, and 3 show the SFR, baryonic mass within $30 \mathrm{kpc}$, and gas fraction within $30 \mathrm{kpc}$ of all the runs. Each panel shows a subset of simulations, selected to explore one parameter. The runs which are both 'not-overheated' (consistent with X-ray observations) and 'quenched' (labeled 'strong $\downarrow$ ' or 'quenched' in Table 1, 

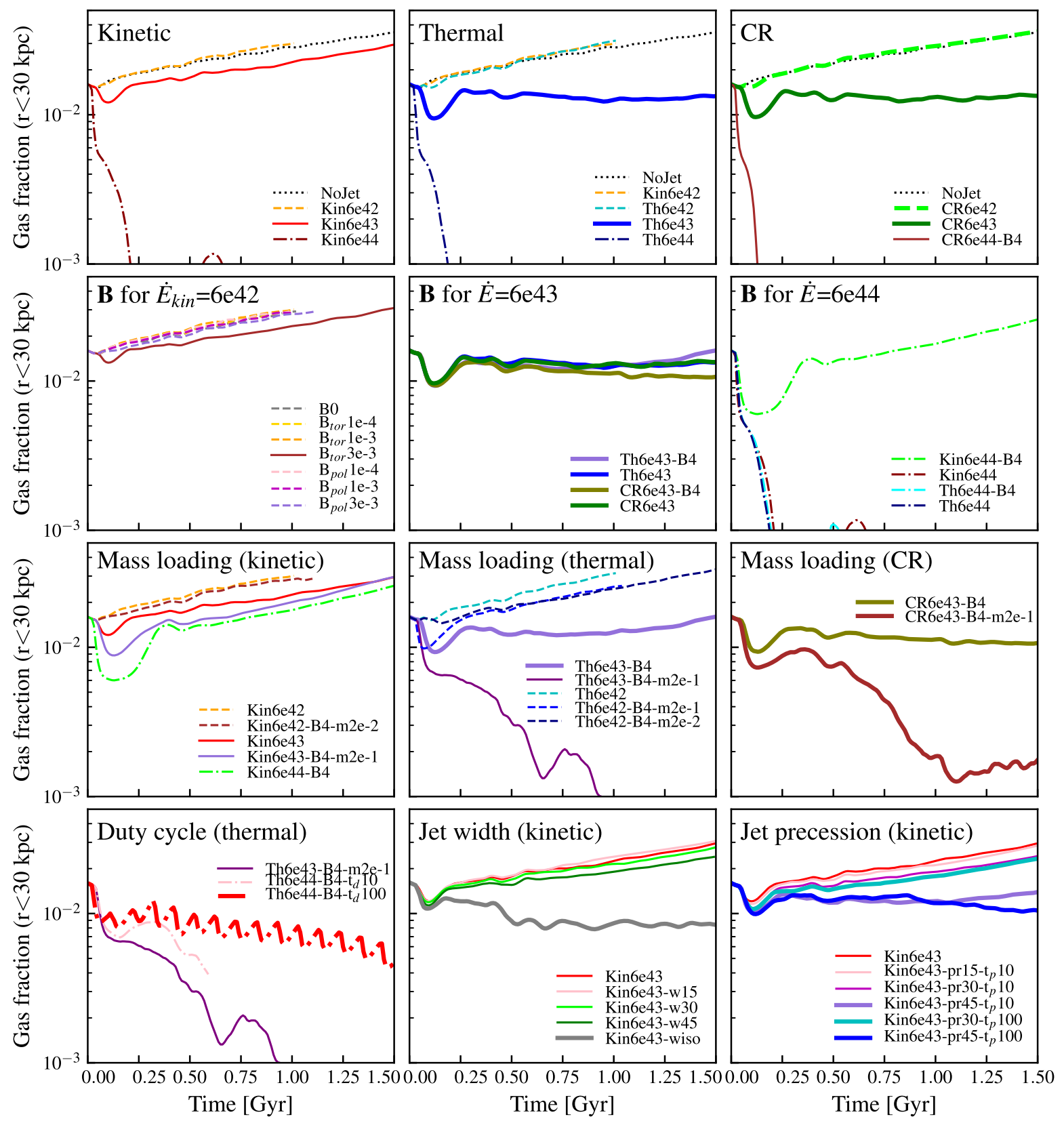

Figure 3. The evolution of the core gas fraction (gas mass over baryonic mass within $30 \mathrm{kpc}$ ) as a function of time. The panels and runs follow the same grid and style as in Fig. 1. Most of the quiescent non-overheated runs (indicated with thick lines) have a core gas fraction that remains almost constant over the duration of the run, indicating an explicit suppression of the gas inflow. The only exception is the lower flux CR jet run ('CR6e42'), where there is still a non-negligible increase of core gas fraction even while the SFR is significantly suppressed.

defined as those with SFR $\lesssim 5 \mathrm{M}_{\odot} \mathrm{yr}^{-1}$ or specific SFR (sSFR) $\lesssim 5 \times 10^{-11} \mathrm{yr}^{-1}$ ) are highlighted with thicker lines. The averaged SFRs of the last $50 \mathrm{Myr}$ of the simulations are also summarized in Table 2 .

Jets with most of their energy in a thermalized component ('thermal jets', for the sake of brevity) can stably quench the galaxies with energy injection rates $\dot{E} \gtrsim 6 \times 10^{43} \mathrm{erg} \mathrm{s}^{-1} \sim 6 \times 10^{-4} L_{\text {Edd }}$ and $\dot{M}=2 \mathrm{M}_{\odot} \mathrm{yr}^{-1}$. If the energy input is higher than $\sim 6 \times 10^{44}$ $\mathrm{erg} \mathrm{s}^{-1}$, the results become explosive - the gas is strongly expelled, and the cores are overheated, qualitatively inconsistent with the Xray observations (see Appendix A). The balance between mass flux and thermal energy loading also changes the results. With the same thermal energy flux, the lower the mass flux (the higher the specific thermal energy), the more efficiently the galaxy is quenched.

With the same averaged thermal energy and mass fluxes, the 'thermal jet' run with a duty cycle reaching $100 \mathrm{Myr}$, 'Th6e44B4- $t_{d} 100$ ', tends to be less explosive than the runs with a shorter duty cycle $(10 \mathrm{Myr})$ or with continuous jets with the same average energy and mass flux. The latter two cases essentially produce the same results.

Cosmic ray dominated jets ('CR jets'), on the other hand, quench much more efficiently. With an order of magnitude lower energy input than is required for thermal jets, $\dot{E} \gtrsim 6 \times 10^{42} \mathrm{erg} \mathrm{s}^{-1} \sim$ $6 \times 10^{-5} L_{\mathrm{Edd}}$, the SFR is significantly suppressed to $\lesssim 3 \mathrm{M}_{\odot} \mathrm{yr}^{-1}$. 


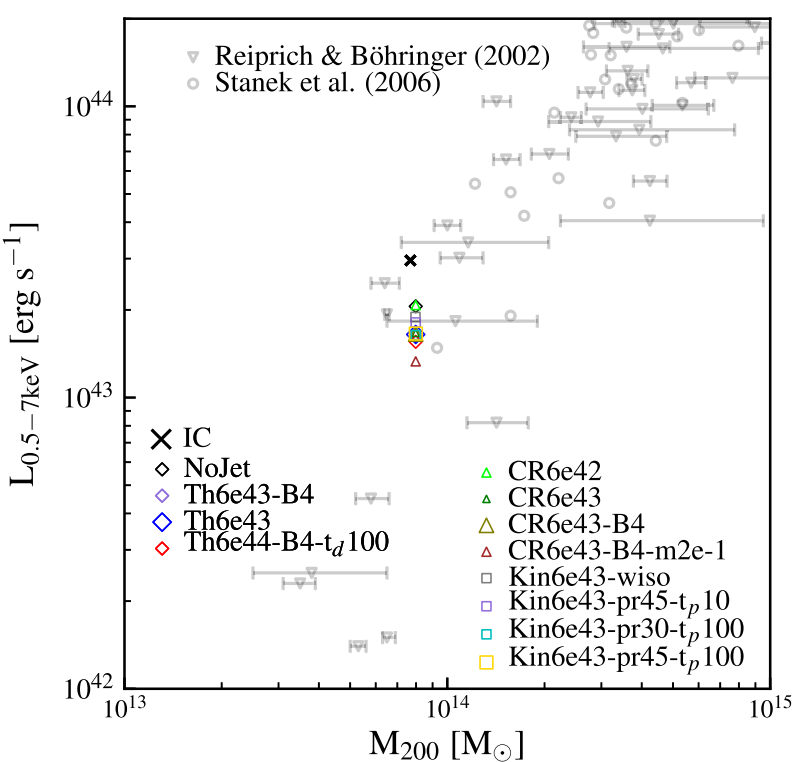

Figure 4. The X-ray luminosity in the $0.5-7 \mathrm{keV}$ band at the end of all the non-overheated quiescent runs. We use $M_{200}$ as the halo mass for our simulations. The lighter markers and the error bars denote the observed values from (Reiprich \& Böhringer 2002; Stanek et al. 2006). We observe very little evolution of the total X-ray luminosity in these runs (compared to the values for the initial conditions). All of the listed runs have X-ray luminosity within the observational range. Note that unless a run is very overheated, its cooling luminosity does not deviate from the initial condition by much. This is true even in the 'NoJet' run, and other 'strong CF' runs. 'Overheated' runs generally have slightly lower X-ray luminosity due to a lower core gas mass, ranging from 1 to $1.5 \times 10^{43} \mathrm{erg} \mathrm{s}^{-1}$ except for 'Th6e44-B4-t ${ }_{d} 10$ ', which has $\sim 2 \times 10^{43} \mathrm{erg} \mathrm{s}^{-1}$.

With $\dot{E} \gtrsim 6 \times 10^{43} \mathrm{erg} \mathrm{s}^{-1}$, the CR jets also quench more efficiently than thermal jets.

Narrow jets with most of their energy in kinetic form ('kinetic jets') quench much less efficiently than CR or thermal jets. With an energy input of $\dot{E} \lesssim 6 \times 10^{43} \mathrm{erg} \mathrm{s}^{-1}$, the SFR is only marginally suppressed. With an even higher energy input $\dot{E} \lesssim 6 \times 10^{44} \mathrm{erg} \mathrm{s}^{-1}$, kinetic jets also do very little to the SFR ('Kin6e44-B4') unless they are significantly widened by strong magnetic fields ('Kin6e44), where the result becomes explosive. This will be discussed in Section 4.3.

Making the kinetic jet wider by construction (at injection) or precessing with a wider angle can potentially help kinetic jets quench more efficiently. However, these effects only matter when the angles are wider than the solid angle affected by an initially narrower jet (30-45 in the case of 'Kin6e43') as we can see in 'Kin6e43-

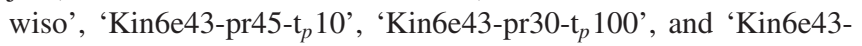
pr45-t $t_{p} 100$ '. Interestingly, longer precession-period jets require a smaller opening-angle to be effective. We will discuss these effects in detail in Section 4.8.

Within the investigated parameter space, making the jet magnetic fields stronger generally has very weak effects, and the most powerful 'magnetic energy dominated' jet we consider has quite a small (factor $\$ 2$ ) effect on the SFR. The only exception is for the very fast kinetic jets, 'Kin6e44', and 'Kin6e44-B4', where the magnetic fields can non-linearly alter the jet propagation and make a difference between having minor effects and causing explosive quenching. The reason for this will be discussed in Section 4.5.
Out of all the investigated runs, 'Th6e43', 'CR6e42', 'CR6e43', 'CR6e43-B4', 'Kin6e43-wiso', 'Kin6e43-pr45-t 10', 'Kin6e43pr45-t $_{p} 100$ ', 'Th6e43-B4', and 'Th6e44-B4-t ${ }_{d} 100$ ' are the runs that both have their SFRs significantly suppressed and avoid being overheated (i.e. are qualitatively consistent with observational gas density and entropy profiles). Interestingly, among the nine runs, the run with $\dot{E}_{\mathrm{CR}}=6 \times 10^{42} \mathrm{erg} \mathrm{s}^{-1}$ ('CR6e42') is the only run with a significantly suppressed SFR which also maintains a steady cooling flow (i.e. growth of the core baryonic mass and core gas fraction). The rest of the runs all have roughly constant core baryonic mass, indicating an explicit suppression of the cooling flow and some switching between 'CC' and 'NCC' haloes. The reason for this will be discussed in Section 4.4.

\subsection{The resulting halo properties of the (non-overheated) quiescent runs}

In this section, we explore in greater depth the runs which manage to regulate star formation without overheating the core. We begin by describing more precisely how we selected these runs.

\subsubsection{How we choose the runs}

We define runs showing regulation as those which are both relatively 'quiescent' (SFR $\lesssim 3 \mathrm{M}_{\odot} \mathrm{yr}^{-1}$ ) and 'non-overheated'. We consider a run to be 'overheated' when it develops a heated core region within $\sim 600$ Myr. We did not use the total X-ray luminosity in the definition because it does not differ as much between runs, which will be discussed in Section 3.2.2. Instead, we base our definition on the entropy and density profiles. The profiles of all the runs are presented in Figs A1 and A2. We plot the profiles of all the 'overheated' runs at the same time, $600 \mathrm{Myr}$, to compare on an equal footing. Given that we are only making a broad comparison of density, temperature, and entropy with the scaled profile (see Section 2.3) and that the central profile is less constrained and may be sensitive to the treatment of point sources, we focus the comparison on radii $\gtrsim 10 \mathrm{kpc}$. We therefore defined the core region to be overheated when (i) the temperature and entropy profiles are significantly above the scaled observed NCC profiles beyond $\sim 10 \mathrm{kpc}$, (ii) there is a clear negative temperature gradient at $r \sim 20 \mathrm{kpc}$, and (iii) there is a greater than 1 dex suppression of the total gas fraction within $30 \mathrm{kpc}$. According to the definition above, 'Kin6e44', 'Th6e44', 'Th6e44-B4', and 'CR6e44-B4' are clearly overheated. 'Th6e43-B4m2e-1', 'Th6e44-B4-t ${ }_{d} 10$ ', and 'CR6e43-B4-m2e-1' are borderline overheated. We still include 'CR6e43-B4-m2e-1' in the plots below, as the heated core develops more slowly and the negative temperature gradient is less dramatic than in the other two cases.

\subsubsection{X-ray luminosities}

The resulting X-ray luminosity of the halo gas is an important constraint for AGN feedback models (e.g. McCarthy et al. 2010; Choi et al. 2015). Fig. 4 shows the predicted X-ray cooling luminosity at the end of all the 'non-overheated' quiescent runs, which are runs with a strong suppression of cooling flows but which do not generate overheated entropy or density profiles (labeled 'strong $\downarrow$ ' or 'quenched' in Table 2), integrated over all gas in the halo, from 0.5 to $7 \mathrm{keV}$.

None of the non-overheated quiescent runs show a significant drop in the X-ray luminosity, indicating that they do not expel a significant amount of the virialized gas within the simulation 

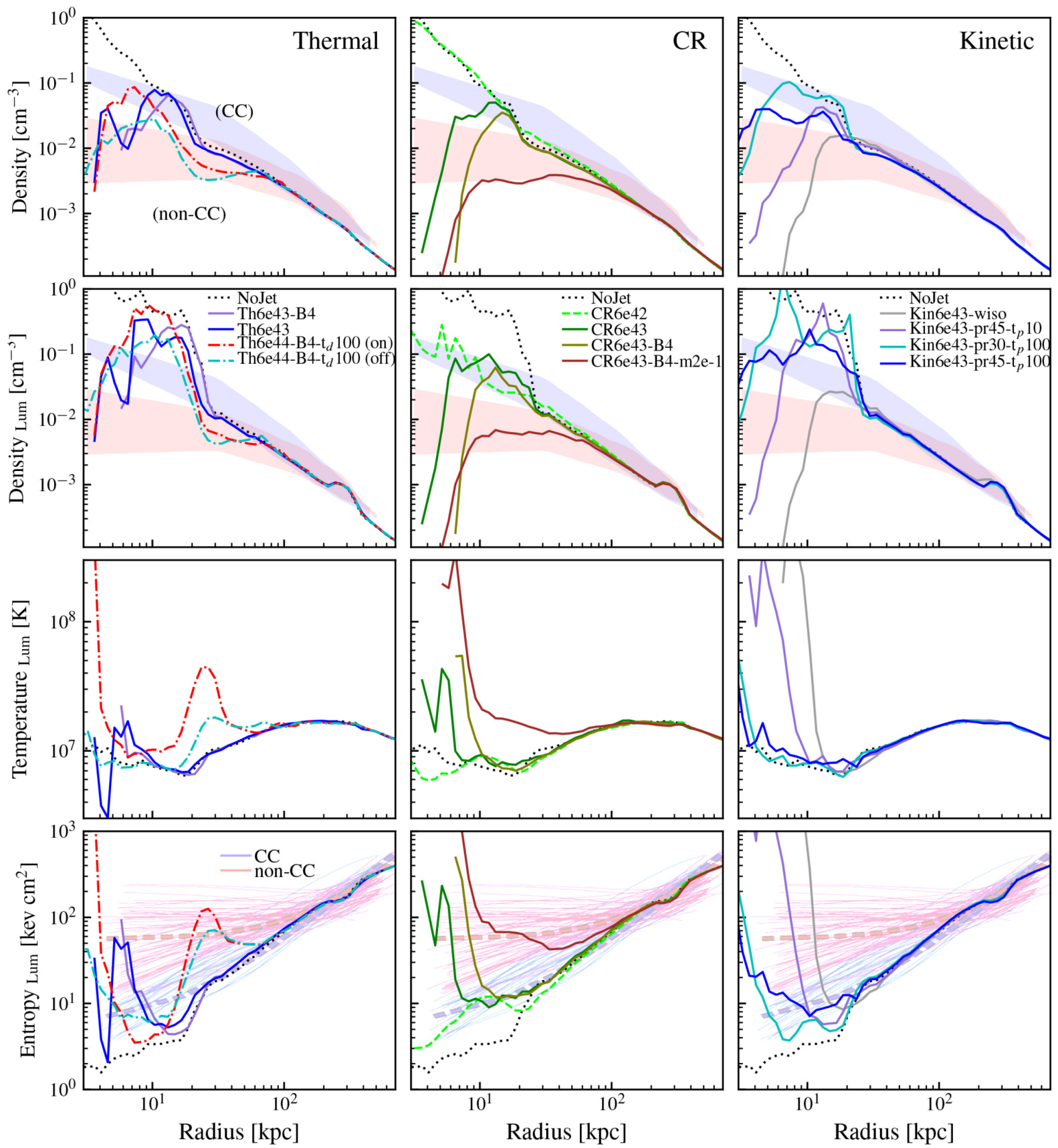

Figure 5. Mean gas density (top row), X-ray cooling luminosity-weighted density (second row), luminosity-weighted temperature (third row), and luminosityweighted entropy (bottom row) versus radius averaged over the last $\sim 50 \mathrm{Myr}$ in the non-overheated quiescent runs from Fig. 1 . The shaded regions in the first and second row and the light curves in the bottom row indicate the observational density and entropy profiles (scaled) for CC (blue) and NCC (red) clusters (McDonald et al. 2013) (scaled to account for the halo mass differences). Almost all of the plotted runs with an energy input of $6 \times 10^{43} \mathrm{erg} \mathrm{s}^{-1}$ have a heated core but are qualitatively within the observational range. The isotropic kinetic input run ('Kin6e43-wiso') has a larger heated region, possibly in tension with the observations. The density and entropy profiles of the run with low-energy CR jets ('CR6e42') and the runs with a long-precession-period kinetic jet ('Kin6e43-pr30- $\mathrm{t}_{p} 100$ ' and 'Kin6e43-pr45- $\mathrm{t}_{p} 100$ ') resemble observed CC clusters. The thermal jet with a 100 Myr duty cycle ('Th6e44-B4- $\mathrm{t}_{d} 100$ ') results in a negative temperature and entropy profile when the jet is at the maximum energy flux, in tension with the observations. The run with a high specific energy CR jet ('CR6e43-B4-m2e-1') resembles the NCC population. The other non-overheated quiescent runs with $6 \times 10^{43} \mathrm{erg} \mathrm{s}^{-1}$ ('Th6e43', 'Th6e43-B4', 'CR6e43', and 'CR6e43-B4') fall between the CC and NCC populations.

time. The X-ray luminosity of all runs is above $\sim 2 \times 10^{43}$ $\mathrm{erg} \mathrm{s}^{-1}$, within the observational range (Reiprich \& Böhringer 2002; Balogh et al. 2006; Stanek et al. 2006; Kim \& Fabbiano 2013; Anderson et al. 2015). Among the runs, the 'CR6e42' run has the highest X-ray luminosity (still within the constraint), consistent with the build up of a large core baryonic mass, as previously noted. We note that the limited variation in the X-ray luminosity is mainly because we start our isolated galaxy simulation from an initial condition resembling a $\mathrm{CC}$ cluster and run it for only $<1.5$ Gyr. 

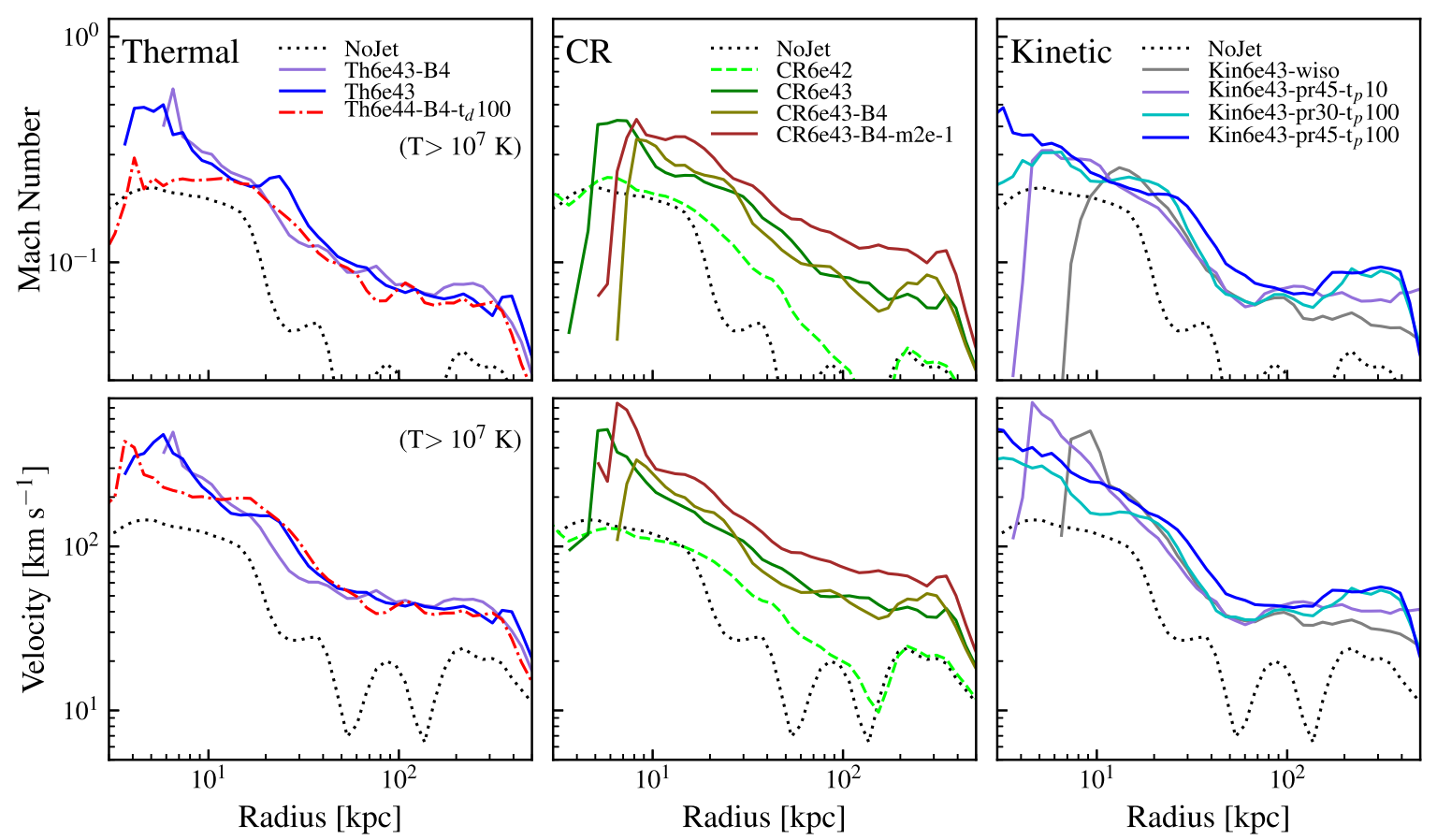

Figure 6. Top row: 1D rms Mach number in gas with $T>10^{7} \mathrm{~K}$ (averaged over the last $50 \mathrm{Myr}$ of the runs) as a function of radius for the non-overheated quiescent runs. Bottom row: 1D rms velocity dispersion for the same gas. (We exclude the radial motion to obtain a rough upper bound on the turbulent velocity.) All thermal and CR jets with $6 \times 10^{43} \mathrm{erg} \mathrm{s}^{-1}$ result in a similar boost of the turbulent velocity, reaching $\lesssim 400 \mathrm{~km} \mathrm{~s}^{-1}$ in the core region, which is slightly higher than the (limited) observational constraints. Kinetic jets with wide opening-angle or precession also boost the turbulent velocities to a similar value, and the velocity boosts extend to larger radii. Long-precession-period jets (100 Myr) further boost the turbulent velocity around 10-70 kpc when the opening-angle reaches $30-40^{\circ}$, but only by a factor of $\$ 2$. The 'CR6e 42 ' run has slightly lower turbulent velocities, roughly $100 \mathrm{~km} \mathrm{~s}^{-1}$, which is broadly consistent with observations of the Perseus cluster (Hitomi Collaboration 2016, 2018).

\subsubsection{Temperature, density, and entropy}

Fig. 5 shows the average density, luminosity-weighted density, temperature, and entropy as a function of radius, averaged over the last 50 Myr for each simulations. We excluded the jet cells themselves from the calculation. The shaded regions in the top and second row indicate the observational density profiles (scaled) for CC (blue) and NCC (red) clusters (McDonald et al. 2013). To account for the difference in halo mass between the observations and our simulations, The observational data are scaled as described in Section 2.3. We emphasize that we focus our comparison on radii $>10 \mathrm{kpc}$, and we define 'overheated' as detailed in Section 3.2.1. The smaller-scale variations within that radius vary in time and should be interpreted only in a very qualitative sense.

Most of the runs with a significantly suppressed SFR also have a lower gas density, and show the presence of heated gas in the core region of the galaxy $(\mathrm{r}<10 \mathrm{kpc})$. 'CR6e42' is, again, the only exception in that neither the temperature nor the density is significantly altered. For the run with a $100 \mathrm{Myr}$ duty cycle, 'Th6e44B4- $t_{d} 100$ ', we plotted the profiles both when the jet is on and when it is off. When the jet is on, we see a quick rise of temperature in the core region of the galaxy. However, there is a delay in density suppression at around $10 \mathrm{kpc}$ as the density there is the highest when the jet is off.

Most of the non-overheated quiescent runs with thermal or CR jets agree reasonably with the observations. The density and entropy profiles in 'CR6e42' end up very much resembling those of observed CC clusters (compare Sanderson, Ponman \& O'Sullivan 2006; Sanderson, O'Sullivan \& Ponman 2009; Hudson et al. 2010; McDonald et al. 2013), while 'CR6e43-B4-m2e-1' resembles a NCC cluster. The other runs ('Th6e43', 'Th6e43-B4', 'CR6e43', and 'CR6e43-B4') fall between the CC and NCC profiles. For the thermal jet run with a 100 Myr duty cycle ('Th6e44-B4-t ${ }_{d} 100$ '), when the jet is on, and at a maximum energy flux, a negative temperature and entropy gradient is observed around $20-60 \mathrm{kpc}$, in possible tension with the observations. Such features die down when the jet is off, and then the run resembles the $\mathrm{CC}$ clusters.

Kinetic jets with long precession period (100 Myr) also agree reasonably well with the observed CC clusters, while the kinetic jet runs with a shorter precession period (10 Myr) have a slightly more extended heated core region and fall in between the CC and NCC populations. Isotropic kinetic input causes a more dramatic density suppression and a sharper temperature increase in the core region, resulting in the most significant tension with the observations among the kinetic jet runs.

\subsubsection{Turbulent Mach number}

Fig. 6 shows the rms 1D turbulent velocity, defined as $v_{\text {turb }} \equiv\left(\left(v_{\theta}^{2}+\right.\right.$ $\left.\left.v_{\phi}^{2}\right) / 2\right)^{1 / 2}$, and the 1D Mach number $\left(v_{\text {turb }} / v_{\text {thermal }}\right)$ for gas hotter than $10^{7} \mathrm{~K}$ as a function of radius, averaged over the last $50 \mathrm{Myr}$ of the runs. We exclude the radial velocity in the calculation due to the contamination of radial outflows and inflows. This yields a rough upper bound on the turbulent velocity. We also exclude the spawned jet cells as well since they, by construction, can have velocities in excess of $3000 \mathrm{~km} \mathrm{~s}^{-1}$.

All thermal jets with $6 \times 10^{43} \mathrm{erg} \mathrm{s}^{-1}$ result in a similar boost in velocity for radii larger than $\sim 30 \mathrm{kpc}$, despite the different duty cycle, energy loading, and mass flux. Beyond this radius, the 
dynamical time is long enough that the duty cycle's effect can be averaged out. We see a very small boost of the turbulent velocity for gas at small radii in the thermal jet run with a duty cycle of $100 \mathrm{Myr}$. The maximum velocity reaches roughly $200 \mathrm{~km} \mathrm{~s}^{-1}$, broadly consistent with observations of the Perseus cluster (Hitomi Collaboration 2016, 2018). On the other hand, the continuous thermal jet with the same average energy flux boosts the turbulent velocity in the core region of the galaxy to $\lesssim 400 \mathrm{~km} \mathrm{~s}^{-1}$, slightly higher than the observations. $^{7}$

The CR jet with $6 \times 10^{43} \mathrm{erg} \mathrm{s}^{-1}$ results in a very similar turbulent velocity boost as the corresponding thermal jet run, and the maximum velocity reaches $\$ 400 \mathrm{~km} \mathrm{~s}^{-1}$. The run with lower CR input, 'CR6e42', has a much lower turbulent velocity $\left(\$ 200 \mathrm{~km} \mathrm{~s}^{-1}\right)$ at all radii, qualitatively consistent with the Perseus observation.

Kinetic jets with a wide opening-angle or with precession also boost the turbulent velocity to $\$ 400 \mathrm{~km} \mathrm{~s}^{-1}$, again, slightly higher than the observations. Comparing with the thermal or CR jet runs with a similar energy input, the velocity boosts of kinetic jets extend to larger radii. Long-precession-period jets (100 Myr) further boost the turbulent velocity around $10-70 \mathrm{kpc}$ when the opening-angle reaches $30-40^{\circ}$, but only by a factor of $\lesssim 2$.

\section{HOW JETS QUENCH}

As shown in the first three panels of Fig. 1, a CR jet quenches star formation more efficiently than a thermal jet with the same energy flux - i.e. CR jets require only about one-tenth of the energy flux as a thermal jet to quench the same galaxy. Kinetic jets are the least efficient at stopping the cooling flow, and only marginally suppress the SFR unless the opening-angles or procession angles are quite wide. In this section, we discuss how different jet parameters affect jet propagation and galaxy quenching. We first provide a simple model in Section 4.1 for the jet propagation and cocoon expansion, which helps us to interpret the results of our numerical experiments. Then we discuss how it applies to each of the cases in the following sections.

\subsection{A simple model for the cocoon expansion and how it impacts quenching}

Despite the different energy forms, the propagation of a jet builds up a pressurized region (cocoon) with the thermal, $\mathrm{CR}$, or magnetic energy it carries at launch or gains through converting its kinetic energy through shocks. This both heats up gas within the cocoon, reversing the cooling and suppressing cooling instabilities (Voit et al. 2015), as well as building up a pressure gradient, slowing down the gas inflow. We found three criteria to successfully quench a halo, which are summarized in Fig. 7. First, the input energy of any form should, at a minimum, offset the gravitational collapse of the cooling gas:

$$
\begin{aligned}
\dot{E}_{\mathrm{min}} & \sim \dot{M}_{\mathrm{cool}} v_{\mathrm{ff}}\left[R_{\mathrm{cool}}\right]^{2} \\
& \sim 10^{43} \mathrm{erg} \mathrm{s}^{-1}\left(\frac{\dot{M}_{\mathrm{cool}}}{100 \mathrm{M}_{\odot} \mathrm{yr}^{-1}}\right)\left(\frac{v_{\mathrm{ff}}\left[R_{\mathrm{cool}}\right]}{300 \mathrm{~km} \mathrm{~s}^{-1}}\right)^{2},
\end{aligned}
$$

where $\dot{M}_{\text {cool }}$ is the cooling rate and $v_{\text {ff }}\left[R_{\text {cool }}\right]$ is the free fall velocity at the cooling radius $\left(R_{\text {cool }}\right){ }^{8}$

\footnotetext{
${ }^{7}$ We reemphasize that Perseus is a much more massive halo $\left(5-6 \times 10^{14}\right.$ $\mathrm{M}_{\odot}$ ), and the velocity above is used only as a reference point.

${ }^{8}$ Here we define the cooling radius ( $R_{\text {cool }} \sim 30-50 \mathrm{kpc}$ ) as the radius within which the cooling time is shorter than our simulation time $(\sim 1-2 \mathrm{Gyr})$.
}

However, if the energy flux is too high, the result will be explosive. This happens at an energy flux of $\dot{E}_{\max }$, in which case the jet bubble expands quasi-isotropically at the escape velocity $\left(v_{\mathrm{esc}}\left[R_{\mathrm{cool}}\right]\right)$ at the cooling radius. It may expand until it has swept out most of the ICM gas. Supposing the energy is purely advective, and the energy loss is negligible, then, the injected energy $\left(\dot{E}_{\mathrm{tot}, \mathrm{J}}\right)$ is related to the outer shell velocity $\left(v_{\exp }\right)$ as

$\dot{E}_{\text {tot, } \mathrm{J}} \sim 4 \pi R_{\text {cool }}^{2} v_{\text {exp }} \times \frac{1}{2} \rho_{R} v_{\text {exp }}^{2} \propto \rho_{R} R_{\text {cool }}^{2} v_{\text {exp }}^{3}$,

where $\rho_{R}$ is the density around the cooling radius. Therefore,

$\frac{\dot{E}_{\max }}{\dot{E}_{\min }} \propto\left(\frac{v_{\text {esc }}\left[R_{\text {cool }}\right]}{v_{\text {ff }}\left[R_{\text {cool }}\right]}\right)^{3} \sim 10-50$,

given that $\dot{M}_{\text {cool }} \propto \pi R_{\text {cool }}^{3} \rho_{R} / t_{\mathrm{ff}} \propto R_{\text {cool }}^{2} \rho_{R} v_{\mathrm{ff}}\left[R_{\text {cool }}\right]$. This gives a roughly one-order-of-magnitude range for the allowed energy flux.

Another important criterion is that the cooling time within the jet cocoon/bubble has to be long enough such that the energy will not be lost before the cocoon reaches the cooling radius, otherwise, the bulk of the gas within the cooling radius will not be affected and the cooling flows persist. The cooling time is roughly $t_{\text {cool }} \sim k T / \bar{n} \Lambda(T)$, where $T$ is the temperature within the jet cocoon/bubble, $\bar{n}$ is the average number density within the cooling radius, and $\Lambda$ is the cooling function. From equation (4), the expansion time is roughly $t_{\text {exp }} \sim R_{\text {cool }} / v_{\text {exp }} \propto \rho_{R}^{1 / 3} \dot{E}_{\text {tot }, \mathrm{J}}^{-1 / 3} R_{\text {cool }}^{5 / 3}$. The cooling time is long enough when $t_{\text {cool }} \gg t_{\text {exp }}$ or

$$
\begin{aligned}
T & \gg \frac{\bar{n} \Lambda(T) \rho_{R}^{4 / 3} R_{\mathrm{cool}}^{5 / 3} \dot{E}_{\mathrm{tot}, \mathrm{J}}^{-1 / 3}}{k} \\
& \sim 10^{8} \mathrm{~K}\left(\frac{\bar{n}}{0.01 \mathrm{~cm}^{-3}}\right)^{7 / 3}\left(\frac{R_{\mathrm{cool}}}{30 \mathrm{kpc}}\right)^{5 / 3}\left(\frac{\dot{E}_{\mathrm{tot}, \mathrm{J}}}{10^{45} \mathrm{erg} \mathrm{s}^{-1}}\right)^{-1 / 3} .
\end{aligned}
$$

The third criterion is that the solid angle affected by the jet cocoon should be wide enough that cooling can be suppressed over a significant fraction of the volume. The propagation of the jet cocoon qualitatively follows momentum conservation in the z-direction (e.g. Begelman \& Cioffi 1989),

$A_{c} v_{z} \bar{\rho} v_{z}=\bar{\rho} \pi R_{\text {cocoon }}^{2} v_{z}^{2}=\frac{1}{2} \dot{M}_{\mathrm{J}} v_{\mathrm{J}}$

and the balance of energy flux in the perpendicular directions,

$2 A_{\mathrm{tot}} v_{R}\left(\frac{1}{2} \bar{\rho} v_{R}^{2}\right)=\left(2 \pi R_{\text {cocoon }} z_{\text {cocoon }}\right) v_{R} \bar{\rho} v_{R}^{2} \beta=\frac{\gamma}{2}\left(\dot{M}_{\mathrm{J}} v_{\mathrm{J}}^{2}\right)$,

where $A_{c}$ is the cross-section of the whole pressurized region, $A_{\text {tot }}$ is the total surface area of the same region, $R_{\text {cocoon }}$ is the radius of $A_{c}, \mathrm{z}$ is the height to which the jet reaches, $v_{R} \equiv d R_{\text {cocoon }} / d t$ and $v_{z} \equiv d z_{\text {cocoon }} / d t$ are the expansion velocities of the pressurized region in the mid-plane and polar directions, $\bar{\rho}$ is the averaged density within the radius out to which the jet reaches, $\dot{M}_{\mathrm{J}}$ is the jet initial mass flux, $v_{\mathrm{J}}$ is the initial jet velocity, $\beta$ is an order-of-unity geometric factor for the surface area of the pressured region, and $\gamma \equiv \dot{E}_{\text {expansion }} / \dot{E}_{\text {kin }} \propto \dot{E}_{\text {tot }, \mathrm{J}} / \dot{E}_{\text {kin }} \equiv f_{\text {kin }}^{-1}$ is the ratio of the energy flux in the perpendicular direction (proportional to the total injected energy $\dot{E}_{\text {tot, J }}$ ) to the injected kinetic energy flux.

From the equations above, we can solve for the time dependence of $R_{\text {cocoon }}$ and $z_{\text {cocoon }}$. In particular, for a fixed $\bar{\rho}, \dot{M}_{\mathrm{J}}, \dot{E}_{\text {tot, J }}$ and time, the opening-angle of the resulting cocoon scales as

$\frac{R_{\text {cocoon }}}{z_{\text {cocoon }}} \propto\left(\frac{\dot{E}_{\text {tot }, \mathrm{J}}}{\dot{E}_{\text {kin }}}\right)^{5 / 12}$ 
Active galactic nucleus (AGN) jets can suppress cooling flows (and therefore quench star formation) in massive galaxies if the following three criteria are met.

\section{Moderate jet energy flux}

Enough energy for cocoon expansion to balance gas cooling, but not so much energy as to exceed escape velocity at $\mathrm{R}_{\mathrm{cool}}$.

$\rho_{R} R^{2} \operatorname{cool} v^{3} \exp \gtrsim \dot{E}_{\text {tot }, \mathrm{J}} \gtrsim \dot{M}_{\text {cool }} v_{\mathrm{ff}}\left[R_{\mathrm{cool}}\right]^{2}$

\section{Long cooling time within jet cocoon} Longer than time to reach $\mathrm{R}_{\mathrm{cool}}$.

$t_{\text {cool }}\left[R_{\text {cool }}\right]>t$ expansion $\left[R_{\text {cool }}\right]$

Can be achieved by thermal or kinetic jets with high specific energy or $\mathrm{CR}$ jets.

\section{Wide jet cocoon}

Width of cocoon at $R_{\text {cool }}$ is enough to suppress the cooling flow over a wide solid angle.

$\left.\frac{R_{\text {cocoon }}}{z_{\text {cocoon }}}\right|_{R_{\text {cool }}} \gtrsim 1$

Can be achieved by jets with a high non-kinetic component, very light kinetic jets, or jets with initially wide angles.

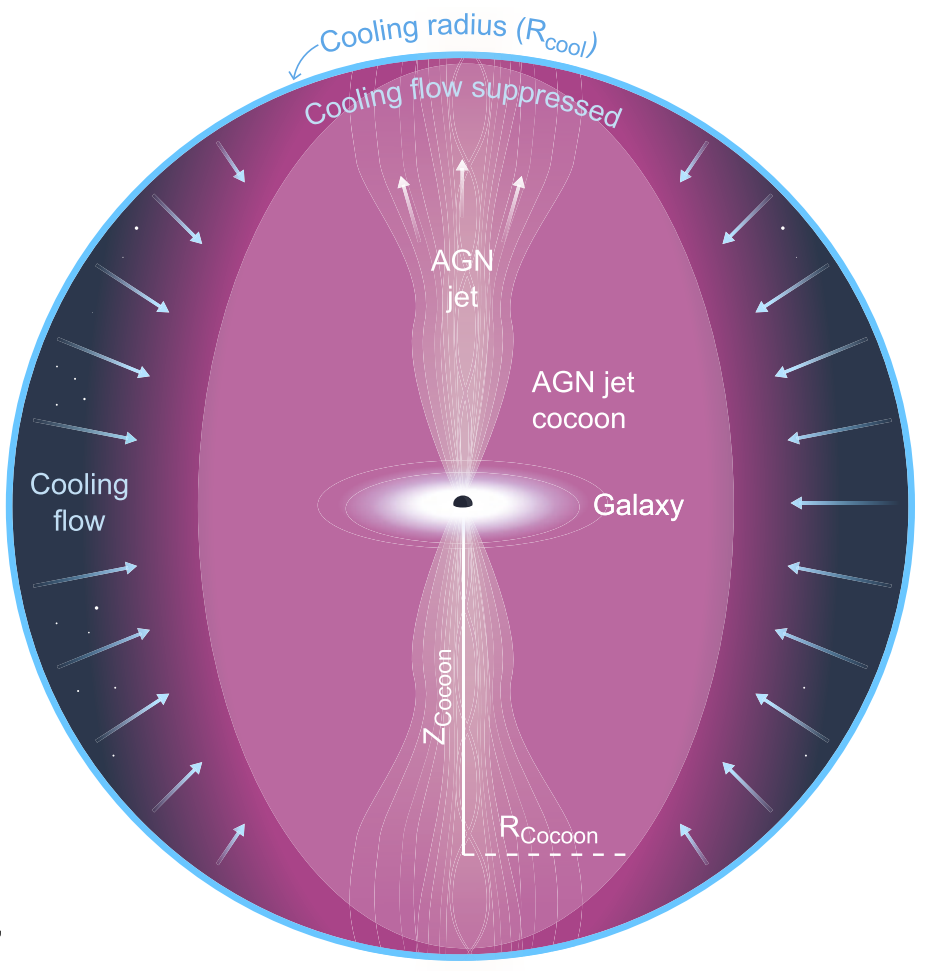

Figure 7. A cartoon picture of the criteria for successful jet models.

On the other hand, for a fixed propagation height $z_{\text {cocoon }}=z_{0}$, the opening-angle scales as

$$
\begin{aligned}
\frac{R_{\text {cocoon }}}{z_{\text {cocoon }}}= & \frac{\gamma z_{0}}{16 \beta}\left(\frac{2 \pi \bar{\rho} v_{\mathrm{J}}}{\dot{M}_{\mathrm{J}}}\right)^{1 / 2} \\
\propto & \begin{cases}\left(\frac{\dot{E}_{\text {tot }, \mathrm{J}}}{\dot{E}_{\mathrm{kin}}}\right)^{3 / 4} & \text { for a fixed } \bar{\rho}, \dot{M}_{\mathrm{J}}, \dot{E}_{\mathrm{tot}, \mathrm{J}} \\
\left(\frac{\dot{E}_{\mathrm{tot}, \mathrm{J}}}{\dot{M}_{\mathrm{J}}}\right)^{3 / 2} & \text { for a fixed } \bar{\rho}, v_{\mathrm{J}}, \dot{E}_{\mathrm{tot}, \mathrm{J}} \\
\left(\frac{v_{\mathrm{J}}}{\dot{M}_{\mathrm{J}}}\right)^{1 / 2} & \text { for a fixed } \bar{\rho}, \dot{E}_{\mathrm{kin}}, \dot{E}_{\mathrm{tot}, \mathrm{J}}\end{cases}
\end{aligned}
$$

Therefore, to have a wider cocoon, the jet needs to have either (i) a smaller kinetic component for fixed total energy and mass flux, (ii) a higher non-kinetic specific energy for fixed jet velocity and total energy flux, or (iii) a lighter but faster jet with fixed total and kinetic energy flux.

At the cooling radius, $R_{\text {cool }} \sim 30 \mathrm{kpc}$, the opening-angle for the jet cocoon is wide enough to be considered quasi-isotropic when $R_{\text {cocoon }} / z_{\text {cocoon }} \gtrsim 1 .{ }^{9}$ As the jet cocoon evolves, it gradually widens and becomes quasi-isotropic (if ever) at a certain height $z_{\text {cocoon }}=z_{\text {iso }}$. If $z_{\text {iso }} \gg R_{\text {cool }}$, the jet can only suppress the inflows within a small solid angle and will not quench the cooling flow, no matter how high the injected energy is. On the contrary, if $z_{\text {iso }} \ll R_{\text {cool }}$, the cooling flow can be suppressed if the energy flux is sufficiently high.

For a purely kinetic jet launched with a very high Mach number, which is always the case,

$\gamma \sim \frac{\rho_{\text {after-shocked }} v_{\text {after-shocked }}^{2}}{\rho_{\text {pre-shocked }} v_{\text {pre-shocked }}^{2}} \sim 1 / 4$.

${ }^{9} R_{\text {cocoon }} / z_{\text {cocoon }}=1$ corresponds to a polar angle of $45^{\circ}$.
The scaling of $z_{\text {iso }}$ generally follows

$$
\begin{aligned}
z_{\text {iso }}= & \left(\frac{\dot{M}_{\mathrm{J}}}{2 \pi \rho v_{\mathrm{J}}}\right)^{1 / 2}\left(\frac{16 \beta}{\gamma}\right) \\
\sim & 23 \mathrm{kpc} f_{\text {kin }} \\
& \times\left(\frac{\dot{M}_{\mathrm{J}}}{2 \mathrm{M}_{\odot} \mathrm{yr}^{-1}}\right)^{1 / 2}\left(\frac{\bar{n}}{0.01 \mathrm{~cm}^{-3}}\right)^{-1 / 2}\left(\frac{v_{\mathrm{J}}}{10^{4} \mathrm{~km} \mathrm{~s}^{-1}}\right)^{-1 / 2} .
\end{aligned}
$$

Therefore, $z_{\text {iso }}<R_{\text {cool }}$ when

$$
\begin{aligned}
v_{\mathrm{J}} \gtrsim & 2 \times 10^{4} \mathrm{~km} \mathrm{~s}^{-1} f_{\text {kin }}^{2} \\
& \times\left(\frac{\dot{M}_{\mathrm{J}}}{2 \mathrm{M}_{\odot} \mathrm{yr}^{-1}}\right)\left(\frac{v_{\mathrm{ff}}}{300 \mathrm{~km} \mathrm{~s}^{-1}}\right)\left(\frac{\dot{M}_{\text {cool }}}{100 \mathrm{M}_{\odot} \mathrm{yr}^{-1}}\right)^{-1},
\end{aligned}
$$

which corresponds to a purely kinetic jet with a very high velocity, or with

$$
\begin{aligned}
f_{\text {kin }} \lesssim & 0.4\left(\frac{v_{\mathrm{J}}}{3000 \mathrm{~km} \mathrm{~s}^{-1}}\right)^{1 / 2}\left(\frac{\dot{M}_{\mathrm{J}}}{2 \mathrm{M}_{\odot} \mathrm{yr}^{-1}}\right)^{-1 / 2} \\
& \times\left(\frac{v_{\mathrm{ff}}}{300 \mathrm{~km} \mathrm{~s}^{-1}}\right)^{-1 / 2}\left(\frac{\dot{M}_{\text {cool }}}{100 \mathrm{M}_{\odot} \mathrm{yr}^{-1}}\right)^{1 / 2},
\end{aligned}
$$

which corresponds to the jet models with more than half of the energy in a non-kinetic form. In the following sections, we discuss how the above scaling relations help explain which jet models quench most efficiently.

\subsection{Kinetic jets transfer energy to larger distances within smaller solid angles}

Kinetic jets quench galaxies mostly through heating and reversing the inflow in the affected region. By shock-heating the gas within 

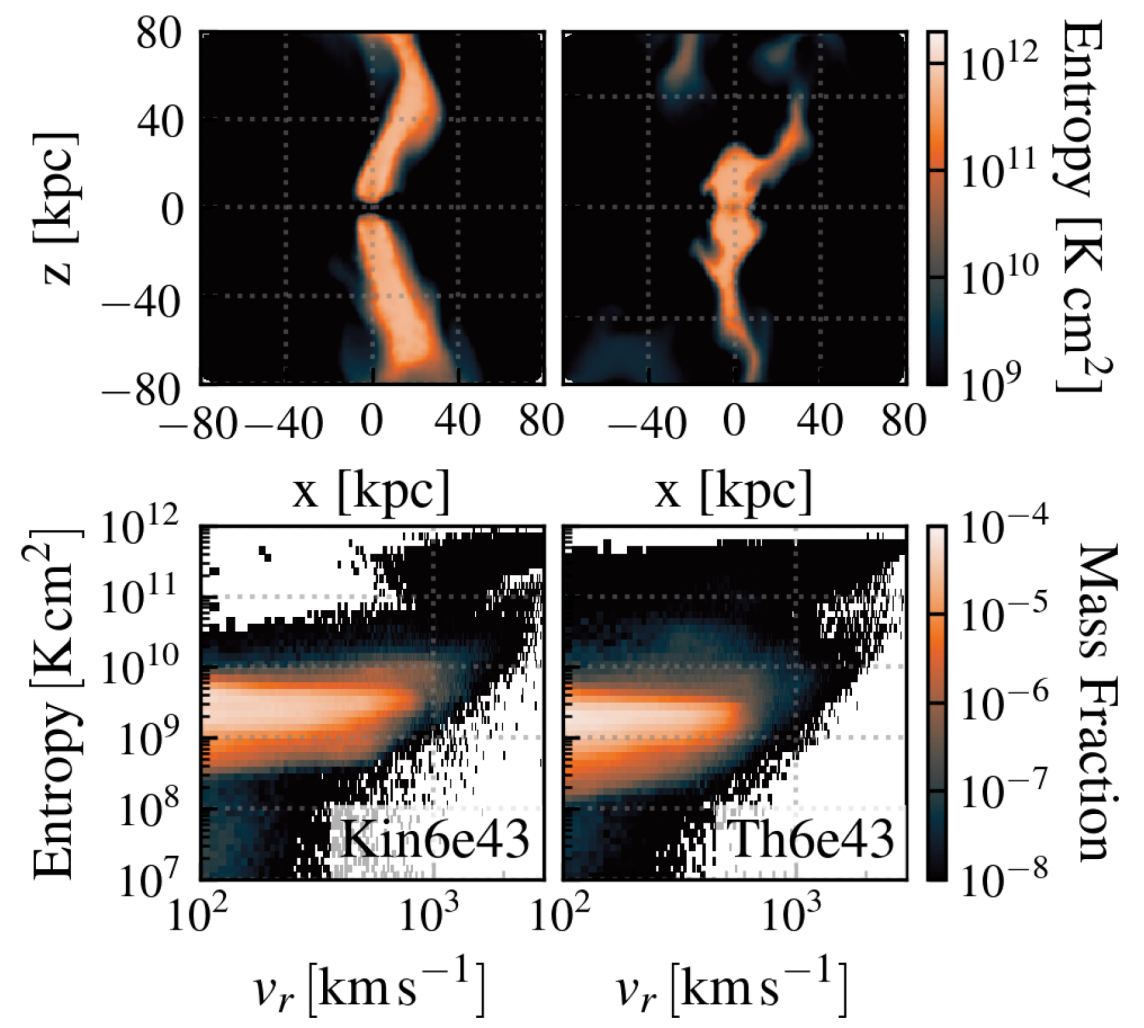

Figure 8. Top row: Entropy distribution of the kinetic jet and thermal jet runs with an equal energy flux of $6 \times 10^{43} \mathrm{erg} \mathrm{s}^{-1}$ (a $\delta y=10 \mathrm{kpc} \mathrm{slice)}$. Bottom row: Entropy and radial velocity distribution. The thermal jet causes a wider heated region in the core $(\leqslant 20 \mathrm{kpc})$, while the kinetic jet propagates more efficiently to a larger radius. Despite the difference in jet velocity and jet temperature, both thermal and kinetic jets show a similar positive correlation between entropy and radial velocity in outflowing gas.

a specific solid angle $\Omega$ (and also transferring momentum/kinetic energy to the gas), they halt the inflows in that cone and suppress cooling flows roughly proportional to $\Omega / 4 \pi$.

In fact, many properties of the outflows in both thermal and kinetic jet runs are very similar despite the very different initial jet temperature and jet velocity. The bottom row of Fig. 8 shows entropy versus radial velocity plots of the thermal and kinetic runs with identical energy fluxes ('Kin6e43' and 'Th6e43'). In both runs, the uppermost disconnected part (at high entropy) consists entirely of spawned jet elements. The kinetic jet elements are shock-heated to a similar entropy and temperature as the thermal jet elements, which are initialized with these values. As expected, the post-shock velocity of the kinetic jet cells is slightly higher than that of the thermal jet cells, but it is important to note that even the "thermal jets' are moving with very large bulk velocity $>3000 \mathrm{~km} \mathrm{~s}^{-1}$ on $\mathrm{kpc}$ scales. Because the energetics and ram+thermal pressure of the jets are similar in both cases, the bulk outflow velocity of the affected ICM is similar in both runs.

Although the properties of entrained, out-flowing gas around the polar direction are similar in these runs, the distribution of inflow versus outflow is quite different away from the poles. Injecting energy in a predominantly kinetic form generally transfers energy out to a large distance from the $\mathrm{BH}$, in a narrower solid angle. As shown in the entropy slices (the top row of Fig. 8), the kinetic jet ('Kin6e43') is narrower, especially at smaller radius, while a thermal jet with the same energy flux ('Th6e43') more effectively heats the core region. This is consistent with equations (13) and (14): an initially narrow kinetic jet $\left(v_{\mathrm{J}}=10^{4} \mathrm{~km} \mathrm{~s}^{-1}\right)$ has an effective opening-angle less than $45^{\circ}$ at the cooling radius ( $\left.\sim 30 \mathrm{kpc}\right)$, while the thermal jet with a small $f_{\text {kin }}$ widens very quickly. 'Kin6e43' also generates a more continuous chimney, indicating a more efficient propagation to large radius. This is expected since the kinetic jets initially have a much higher velocity. Accordingly, kinetic jets mostly invert the inflow within a confined solid angle and only reduce the cooling flow proportionally. On the other hand, thermal jets more uniformly heat up the core region $r \lesssim$ $30 \mathrm{kpc}$ and more effectively quench. Whether the substantially lesscollimated thermal jets are theoretically and observationally realistic remains to be determined.

\subsection{Width of the jet cocoon determines how efficiently it quenches}

Given that the effects of the kinetic jets are limited to a relatively small solid angle, the value of the solid angle can determine how effective the jet is at quenching. Keeping a similar solid angle but increasing the energy (or momentum) flux, on the other hand, has much smaller effects. To produce a kinetic-jet-inflated cocoon with a wide enough solid angle to suppress the cooling flows, we either need a jet with $v_{\mathrm{J}} \gg 2 \times 10^{4} \mathrm{~km} \mathrm{~s}^{-1}$ (following equation 13), or a jet that is initialized with a wide opening-angle. Exploring the former possibility is limited by the maximum jet velocity we can adopt in our MHD simulations.

As we can see in the 'Mass loading (kinetic)' panel of Figs 1 and 2 , despite very different jet velocities, specific energies, and initial jet opening-angles, 'Kin6e43', 'Kin6e44-B4', 'Kin6e43-w15', 'Kin6e43-w30', and 'Kin6e43-w45' share very similar core baryonic mass growth and SFRs. 'Kin6e44-B4' and 'Kin6e43-wiso', on the 

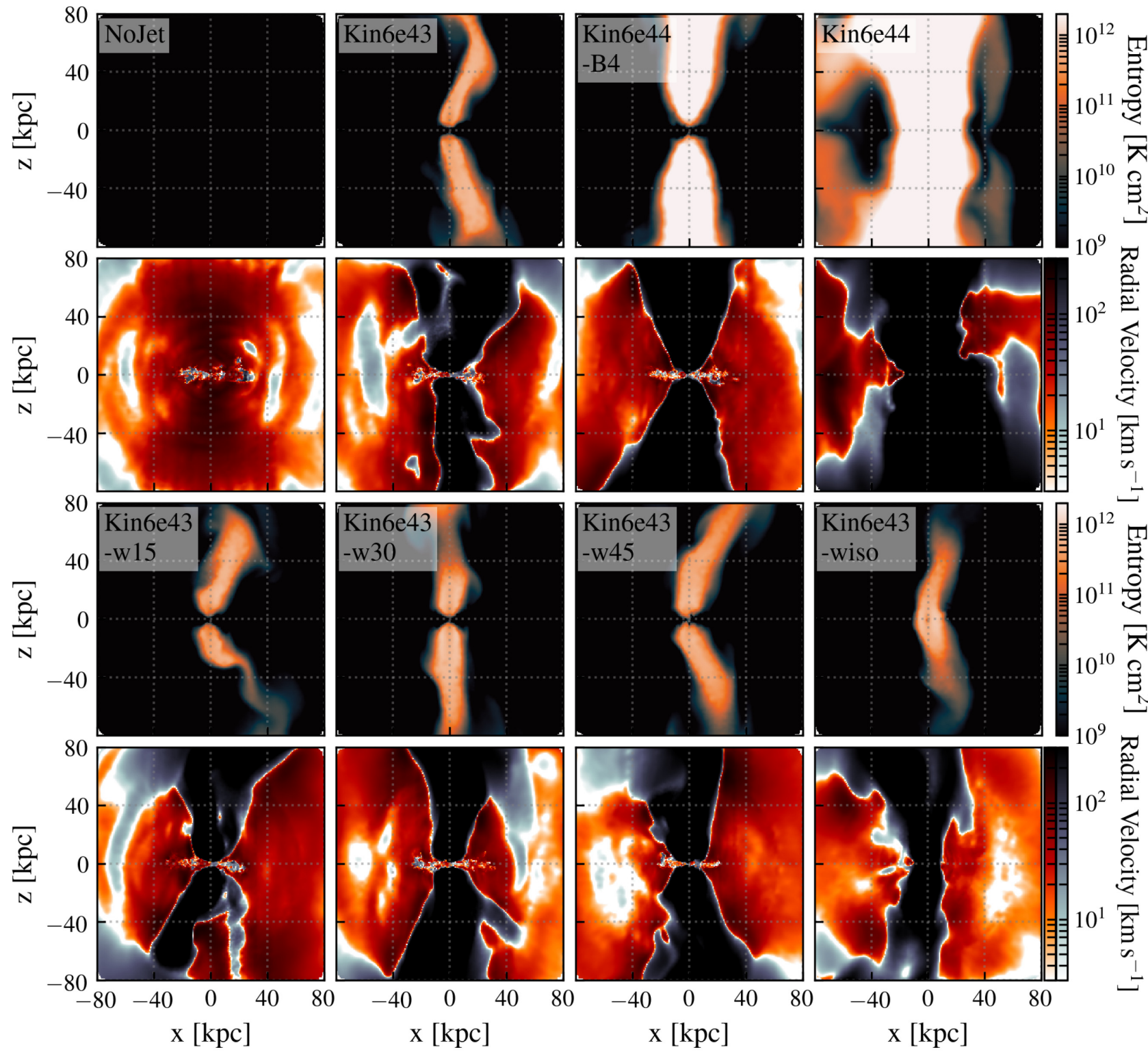

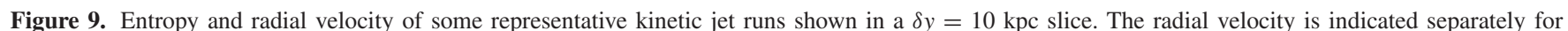

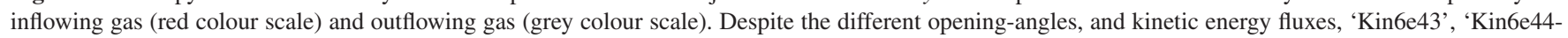

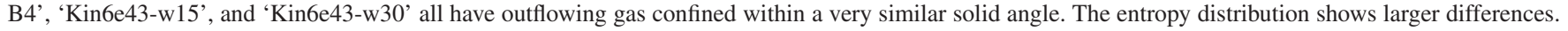
'Kin6e44' has a significantly wider opening-angle due to the magnetic fields, which is explained in Section 4.5.

other hand, are quenched and have lower core baryonic mass due to the larger opening-angle of the jet-inflated cocoon.

Fig. 9 shows the distributions of the entropy (first and third row) and the radial velocity (second and fourth row) of these seven runs and the 'NoJet' run. Inflowing and outflowing velocities are indicated by red and grey colour scales, respectively. Fig. 10 shows the inflow/outflow rates along different angles as a function of polar angle. It is clear that although the entropy distribution differs from run to run, the velocity structures of the runs with similar baryonic inflows ('Kin6e43', 'Kin6e44-B4', 'Kin6e43-w15', 'Kin6e43-w30', and 'Kin6e43-w45') look quite similar. In the 'NoJet' case, gas is inflowing in all directions. In the five aforementioned runs, on the other hand, the velocity is all outflowing at small polar angle (closer to the jet), while at large polar angle (closer to the mid-plane), the velocity is all inflowing. This transition happens at $30^{\circ}-40^{\circ}$ for all of the four cases. As labeled on the plot, the angle is roughly consistent with the angle derived from equation (10) at $r=30 \mathrm{kpc}$ assuming $n \sim 0.01 \mathrm{~cm}^{-3}$. Even the 'Kin6e43-wiso' run, despite the isotropic injection, is still collimated by the surrounding over-density in the midplane of the galaxy. However, the inflow at the core region of this run is shut down isotropically, consistent with the zero SFR at a later time. In contrast, 'Kin6e44' has a much wider region that is dramatically outflowing. The reason for this is that the jet has a non-thermal component (in this case, magnetic) with comparable energy to the kinetic component, producing rapid broadening of the cocoon.

In Fig. 10, the inflows and outflows are calculated in a shell of 25-35 kpc, somewhat inside the cooling radius. ${ }^{10}$ We clearly see that

${ }^{10} \mathrm{We}$ note that the plotted values will vary if we choose a different radius, but the conclusions remain the same. 

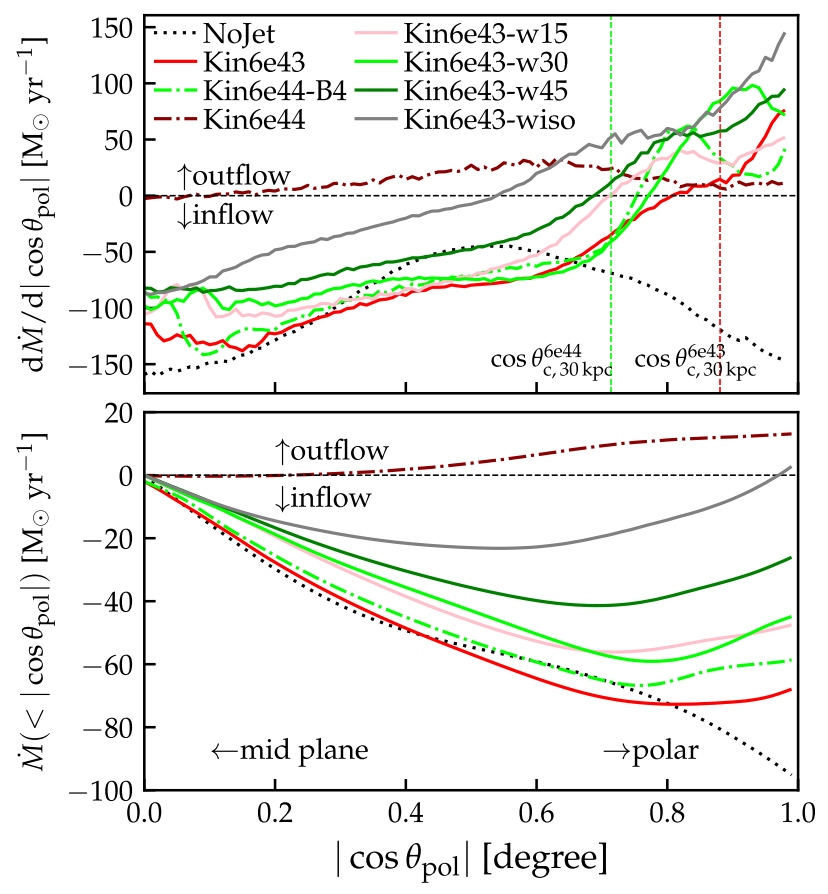

Figure 10. Top: The distribution of radial gas inflow $(\dot{M}<0)$ and outflow $(\dot{M}>0)$ as a function of the cosine of the polar angle $\left(\left|\cos \theta_{\text {pol }}\right|\right)$ calculated in a shell of $25-35 \mathrm{kpc}$ averaged over the last $50 \mathrm{Myr}$ of each run. Bottom: The integrated value of the mass flux from $\left|\cos \theta_{\text {pol }}\right|=0$ to $\left|\cos \theta_{\text {pol }}\right|$. The vertical lines label the corresponding cocoon opening-angle at $30 \mathrm{kpc}$ for the 'Kin6e43' and 'Kin6e44-B4' runs according to equation (10). Without jets, there is net inflow in all directions. With kinetic jets, we generically see outflow along the polar directions and inflow in the mid-plane, with net (angle-integrated) inflow. The outflow opening-angle increases as we increase the effective jet opening-angle, but even isotropic jets ('Kin6e43-wiso') are collimated by the inflowing halo gas and feature inflows within $\sim 30^{\circ}$ of the mid-plane (only the most violent jet here produces isotropic outflows).

runs that produce a wider opening-angle outflow 'cocoon' or bubble in Fig. 9, and thus have outflow along a broader range of angles, have lower integrated inflow rates, which is consistent with their accreted baryonic mass and SFRs in Figs 1 and 2.

In brief, the jet drives shocks which inflate a cocoon, directly impacting gas within an opening angle that depends on the form of the jet. Beyond this effective opening-angle, the cooling flows are much less affected. As a result, increasing the kinetic energy input does not necessarily mean more effective quenching if the affected solid angle is not enlarged because the maximum effect is expelling all the gas in that cone. Consistent with this, the 'Kin6e44B4' run has very similar inflow and outflow structures as 'Kin6e43' in Fig. 10 despite having an order of magnitude higher kinetic energy flux. However, enlarging the initial kinetic jet opening-angle while keeping the total energy flux the same can potentially make the jet quench the galaxy more efficiently.

Remarkably, if we use equation (12) to estimate the scaling of the cocoon opening-angle with the jet $\dot{M}$ and $\dot{E}$ or $v_{\mathrm{J}}$, we obtain the same scaling for opening-angle as seen in Fig. 10 for the initially 'narrow' jets (vertical dashed lines).

\subsection{Why the CR jet quenches star formation more efficiently}

We find that CR-dominated jets quench more efficiently, and potentially more stably, than thermal, kinetic, and magnetic jets. We argue that this is due to three factors: (i) CR pressure support, (ii)

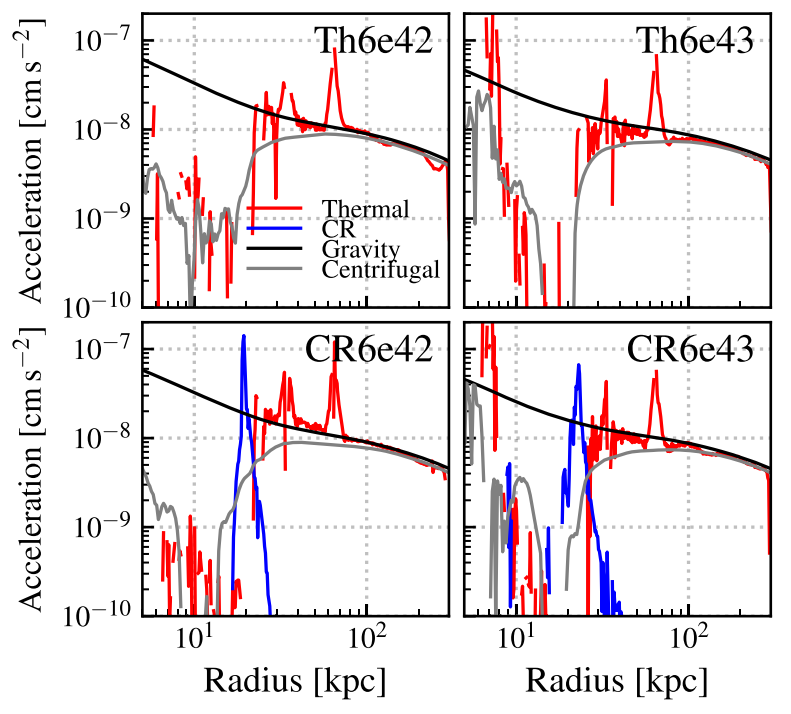

Figure 11. Comparison of gravitational, rotational, thermal pressure and CR pressure gradient acceleration. The centrifugal acceleration is defined as $G M_{\text {enc }} / r^{2}-v_{\text {rot }}^{2} / r$. In the core region, where cooling is rapid, the thermal pressure gradient is not outward and support is lost. In our CR runs, the CR pressure gradient predominantly balances gravity in the core region.

modification of the thermal instability, and (iii) CR propagation. Injected CRs provide pressure support to the gas and have long cooling times, which leads to the formation of a CR pressuredominated cocoon. Because the CR energy density is much larger than kinetic energy density, the CR jet cocoon covers a wider angle (as expected), and can therefore more efficiently suppress inflow. If the CR losses are negligible and CRs become quasi-isotropic, with an effective isotropically averaged diffusivity $\tilde{\kappa}$ (which includes streaming+advection), then as shown in various studies (Butsky \& Quinn 2018; Hopkins et al. 2019, 2021a, b, c; Ji et al. 2020) the CR pressure for steady-state injection is $P_{\mathrm{CR}}(r) \sim \dot{E}_{\mathrm{CR}} / 12 \pi \tilde{\kappa} r$. Comparing the outward acceleration $\rho^{-1} \nabla P$ to gravity $\left(\sim v_{c}^{2} / r\right)$, where $v_{c}$ is the circular velocity, CR pressure alone can support the gas if

$$
\begin{aligned}
\dot{E}_{\mathrm{CR}} \gtrsim & 10^{43} \mathrm{erg} \mathrm{s}^{-1} \\
& \left(\frac{10^{29}}{\tilde{\kappa}}\right)\left(\frac{n_{\mathrm{gas}}}{0.01 \mathrm{~cm}^{-3}}\right)\left(\frac{r}{30 \mathrm{kpc}}\right)\left(\frac{v_{c}}{500 \mathrm{~km} \mathrm{~s}^{-1}}\right)^{2}
\end{aligned}
$$

(where we recall the cooling radius is $\sim 30 \mathrm{kpc}$ here and the diffusivity used here is $10^{29} \mathrm{~cm}^{2} \mathrm{~s}^{-1}$; see Chan et al. 2019; Hopkins et al. 2019, 2021c). This roughly explains the required CR energetics we find in our simulations, as well as the radii/densities where CR pressure dominates for a given $\dot{E}_{\mathrm{CR}}$. This is also consistent with the comparison of the total centrifugal acceleration and the acceleration due to CR and thermal pressure gradient as shown in Fig. 11. ${ }^{11}$

CR-dominated jets can also help quench by modifying the nonlinear behaviour of the thermal instability, as suggested in Ji et al. (2020) and shown rigorously in Butsky et al. (2020). In brief: if CR pressure balances gravity and dominates over thermal pressure in a thermally unstable medium, then cooling gas follows total pressure equilibrium (not just thermal pressure equilibrium) and cooling gas can remain diffuse (rather than being compressed to high densities; i.e. the cooling changes from isobaric to isochoric), as shown in Fig. 12. This, in turn, slows the 'precipitation' of dense, cold gas

\footnotetext{
${ }^{11}$ We used the median pressure (weighted by mass) in each radial bin to calculate the pressure gradient in Fig. 11 to better show the difference.
} 

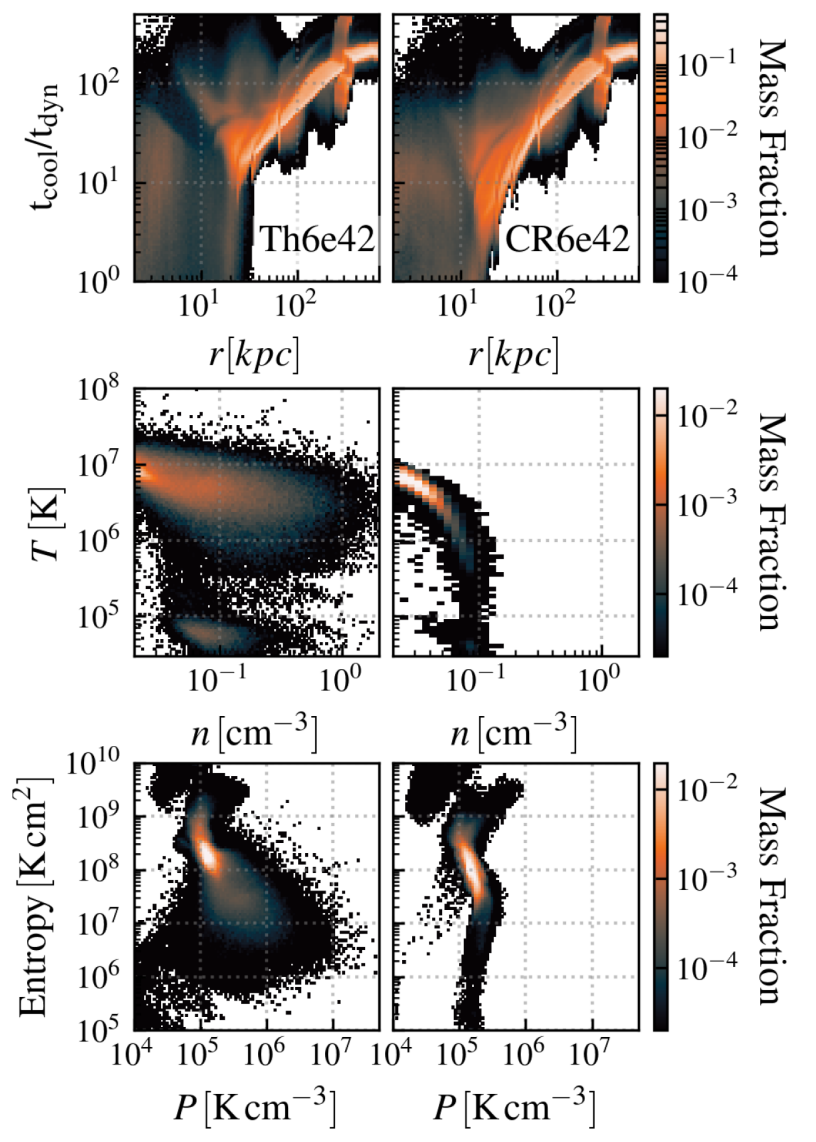

Figure 12. Ratio of the cooling time to dynamical time $\left(t_{\text {cool }} / t_{\text {dyn }}\right.$ ) (top row) and the phase structure for $15-30 \mathrm{kpc}$ in the temperature-density plane (middle row) and entropy-pressure (thermal $+\mathrm{CR}$ ) plane (bottom row) for the thermal and CR jet runs. The plots are averaged over $0.95-1$ Gyr of each run. In the CR runs ('CR6e 42 '), there is more warm, thermally unstable gas residing at intermediate densities (and at $\sim 10-30 \mathrm{kpc}$ ) without accreting (e.g. our 'CR6e42' run) and the formation of the dense cold phase appears somewhat delayed. The CR jet run also has gas following a constant total pressure path with narrow density variation while cooling. In the thermal jet run, there is generally a wider density distribution.

from the cooling flow that would otherwise accrete (Voit et al. 2017), allowing it to instead remain diffused and supported by CR pressure. We see indirect evidence for this (in Fig. 12) in our CR runs as more warm thermally unstable gas resides at intermediate densities (and at $\sim 10-30 \mathrm{kpc}$ radii) without accreting (e.g. our 'CR6e42' run) and formation of the dense cold phase appears somewhat delayed. There is also a slight thermal instability difference in the CR jet runs with different fluxes. We discuss this further in Appendix B.

Heating from CRs plays a smaller role in quenching. For all of our $\mathrm{CR}$ jet runs, $\mathrm{CR}$ collisional heating and streaming heating contribute at most $1 / 20$ and $1 / 6$ of our CR injection rate, respectively. This amount of heating should not have a major effect on quenching, as we can see in the thermal jet runs with such a corresponding energy flux.

The ability of CRs to stream or diffuse (i.e. large $\tilde{\kappa}$ above) relative to the gas is crucial to these behaviours. If CRs were purely advected with the gas, they would simply represent slowly-cooling internal energy. But because CRs can stream through gas, their pressure profile operates akin to a fixed background, which means that if we increase $\dot{E}_{\mathrm{CR}}$ further, the behaviour is not 'explosive.' Specifically, as shown in Hopkins et al. (2021a), even if $\left|\nabla P_{\mathrm{CR}}\right| \gg \rho\left|\mathbf{a}_{\mathrm{grav}}\right|$, then although $\mathrm{CR}$ pressure is sufficient to drive gas outflows,

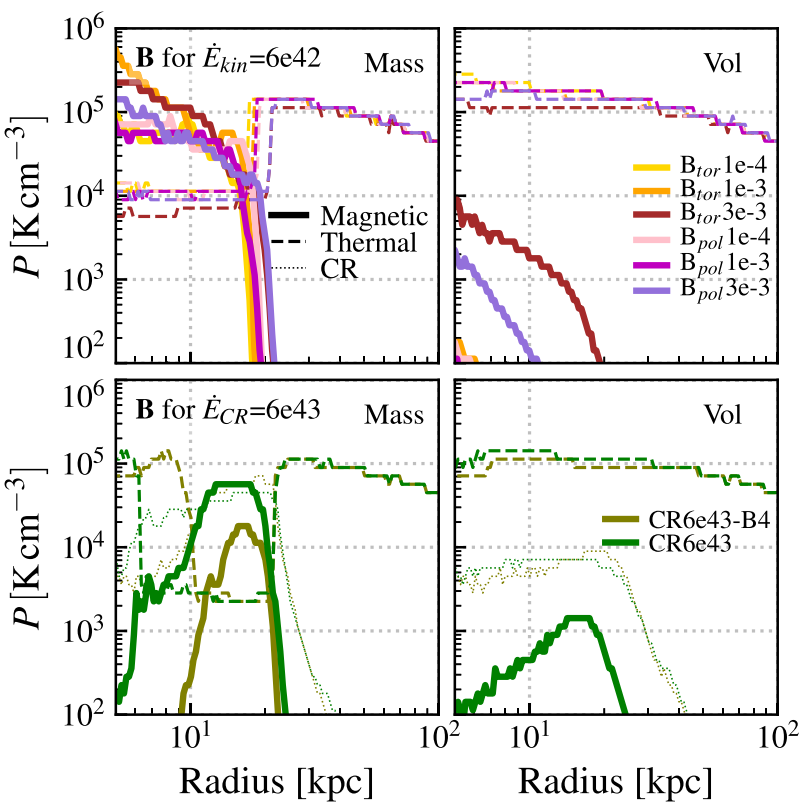

Figure 13. The volume and mass-weighted thermal (thin dashed), magnetic (thick solid), and CR (thin dotted if applicable) pressure profiles for runs with different jet magnetic field variations. The magnetic pressure can only be comparable to the thermal pressure in the $10-30 \mathrm{kpc}$ range and then, only in dense cool gas, given that the volume-weighted values are much lower than the mass-weighted ones. At radius $\gtrsim 100 \mathrm{kpc}$ (where jet effects are weak), or wherever the B-fields are a large fraction of the total pressure (i.e. appear to have saturated), $|\bar{B}|$ is weakly sensitive to the injected fields.

these outflows are weak. Independent of $P_{\mathrm{CR}}$ or $\dot{E}_{\mathrm{CR}}$, they rapidly accelerate gas up to a terminal velocity $\sim V_{c}$, i.e. trans-sonic with respect to the hot halo gas, which then 'coasts' in the outer halo and beyond. In comparison, a conventional continuous-injection pressure-driven blastwave generally produces hypersonic outflows (and accelerates more rapidly in a declining density profile as is typical of outer haloes). CR diffusion is more effective than thermal conduction (except at high temperature), making both 'CR6e44' and 'CR6e43-B4-m2e-1' less overheated than the corresponding 'Th6e44' and 'Th6e43-B4-m2e-1', as shown in Figs A1 and $\mathrm{A} 2$.

\subsection{The effects of magnetic fields}

Magnetic fields usually only have limited effects (factor $\lesssim 2$ ) in quenching the galaxy or suppressing cooling flows. The exception is 'Kin6e44', where the galaxy is quenched while an otherwise identical simulation with an order of magnitude lower magnetic field strength ('Kin6e44-B4') has strong cooling flows and high SFRs.

In 'Kin6e44', the magnetic energy input is $\sim 2-3 \times 10^{44} \mathrm{erg} \mathrm{s}^{-1}$, similar to the kinetic energy input. We therefore expect the nonkinetic pressure to broaden the jet cocoon (equation 14) and indeed, we see a much wider jet cocoon that produces a wide-angle outflow. In other runs, the highest magnetic energy input rate is $\sim 10^{43} \mathrm{erg} \mathrm{s}^{-1}$, insufficient to strongly broaden the cocoon.

The direct effect of gas acceleration by magnetic pressure is weak. Magnetic pressure is only high in the core region and dense structures, as shown in Fig. 13 and Fig. 14. The magnetic pressure can be higher than the thermal pressure within $\sim 20 \mathrm{kpc}$ if mass-weighted but is always subdominant to thermal pressure if volume-weighted. This indicates that magnetic field strengths are only high in the dense 


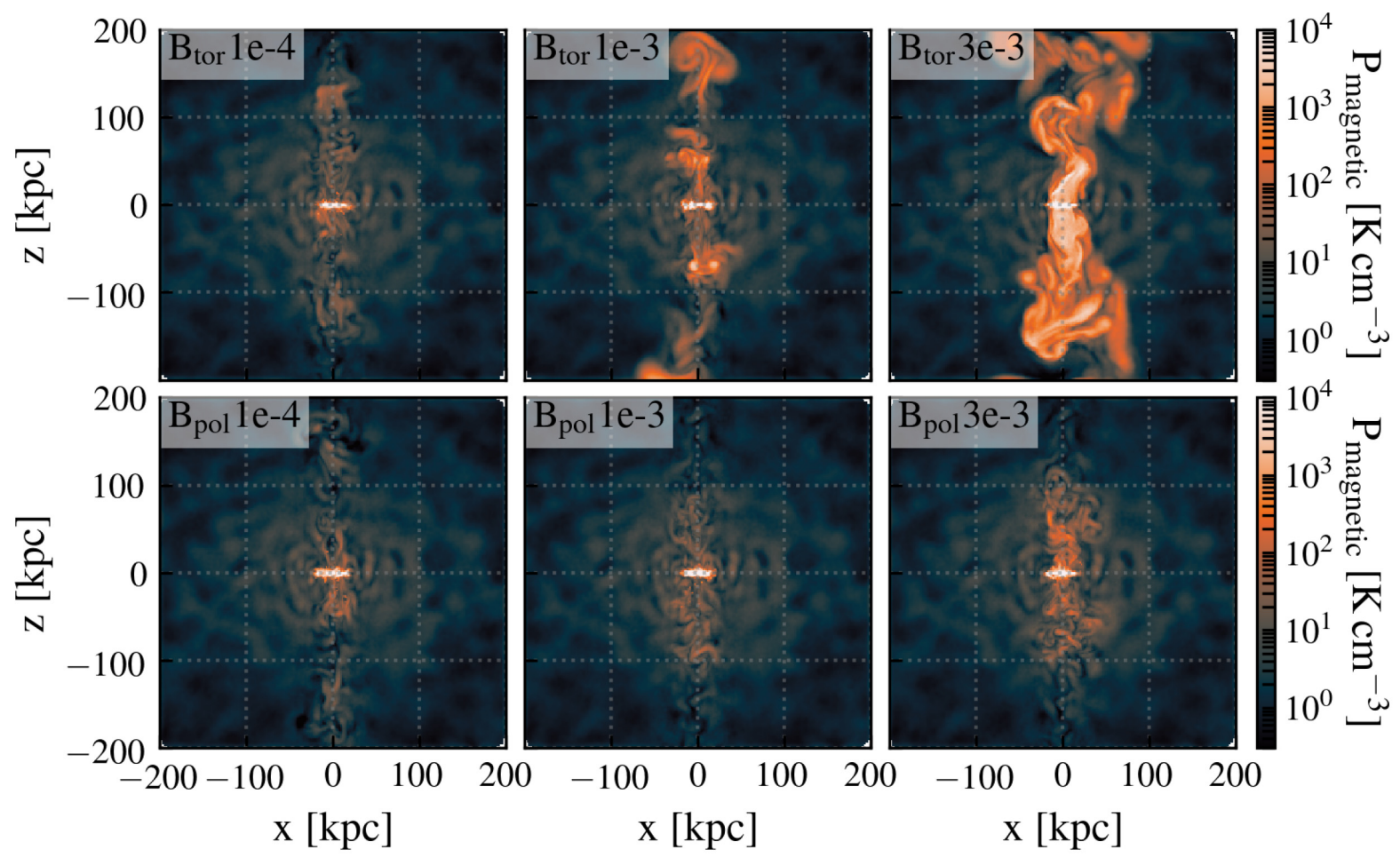

Figure 14. The magnetic pressure morphology shown in a $\delta y=10 \mathrm{kpc}$ slice of the runs with the same kinetic jet, $6 \times 10^{42} \mathrm{erg} \mathrm{s}^{-1}$, but with different jet magnetic fields. Beyond $\sim 30 \mathrm{kpc}$, the magnetic pressure is high only close to the $z$-axis, where the gas is directly affected by the jet. With similar initial magnetic field strengths, a jet with initially toroidal magnetic fields is able to maintain larger magnetic field strengths further along the jet axis, compared to a jet with poloidal magnetic fields.

cooler gas, and in that gas the B-fields appear saturated (independent of injected field strengths). Moreover, in those dense structures, the cooling is already effective, and the extra magnetic pressure cannot do much. In the regions where the density is relatively low, the magnetic pressure support is less important.

Outside of these dense regions, we do see slightly broader cocoons (hence more efficient quenching) for jets with toroidal (versus poloidal) fields, at the same initial strength (compare e.g. ' $\mathrm{B}_{\text {tor }} 1 \mathrm{e}-4$ ' and ' $\mathrm{B}_{\text {pol }} 1 \mathrm{e}-4$ ' in Fig. 14). This is most likely due to toroidal fields being less of an impediment to perpendicular cocoon expansion. This difference may also partially result from enhanced amplification, as the galactic field is also toroidal near the injection site.

\subsection{Mass flux and energy loading}

We also tested how the cooling flows and SFRs differ when using the same total energy in a given form but with different mass flux and energy loading. Given a fixed total energy, the lower the mass flux (i.e. the higher the specific energy of the jet), the more effective the quenching is, as expected from simple analytic predictions of how rapidly the cocoon can inflate (equations 10 and 14 ).

As we can see in Fig. 1, 'Th6e43-B4-m2e-1' quenches more effectively than 'Th6e43-B4'. The former jet has an order of magnitude lower mass flux than the latter despite having the same thermal energy flux. 'Th6e42-B4-m2e-2' also quenches slightly more efficiently than 'Th6e42-B4-m2e-1' and 'Th6e42'. The major difference between jets with different specific thermal energies and mass fluxes is the cocoon width, as shown in Fig. 15, especially in the $6 \times 10^{43} \mathrm{erg} \mathrm{s}^{-1}$ runs. 'Th6e-43-B4-m2e-1' has a wider solid angle for which the gas is outflowing than for the 'Th6e43-B4' run. We see the same comparing CR runs ('CR6e43-B4-m2e-1' and 'CR6e43-B4'). In all of these cases, the width of the low-density evacuated cocoon scales roughly $\propto\left(\dot{E}_{\text {tot, } \mathrm{J}} / \dot{M}_{\mathrm{J}}\right)^{3 / 2}$, as expected from equation (10). The effects in the low-energy thermal jet runs or pure kinetic runs are weaker, as the cooling flows are not strongly suppressed (the cocoon has insufficient energy to grow). As shown in equation (13), a purely kinetic jet with $\dot{M}=2 \mathrm{M}_{\odot} \mathrm{yr}^{-1}$ needs a velocity $>2 \times 10^{4} \mathrm{~km} \mathrm{~s}^{-1}$ to have a sufficient width at the cooling radius.

\subsection{Duty cycle}

We also tested the difference between a jet with constant mass and energy flux and jets with various duty cycles but the same averaged mass and energy flux. We found that the run with a $\sim 100 \mathrm{Myr}$ episodic period and a $\sim 10$ per cent duty cycle ('Th6e44-B4- $t_{d} 100$ ') is less effective than the run with a shorter period and the same duty cycle ('Th6e44-B4-t ${ }_{d} 10$ ') or the continuous run 'Th6e43-B4-m2e-1'. The two latter runs turn out to be very similar, as shown in Fig. 16. 'Th6e44-B4-t ${ }_{d} 100$ ', on the other hand, has layers of inflows and outflows indicating different episodes of the jet. As shown in Fig. 2, the core baryonic mass of 'Th6e44-B4- $\mathrm{t}_{d} 100$ ' takes roughly $100 \mathrm{Myr}$ to recover in each duty cycle while remaining overall constant on average. In comparison, the dynamical time at the region where multiphase gas starts to form $(\sim 30 \mathrm{kpc})$ is roughly $10 \mathrm{Myr}$ and the cooling time for the hot gas at the same radius is $\gtrsim 100 \mathrm{Myr}$, so the cooling flow has time to recover when the jet is off. On the contrary, in the run with a $10 \mathrm{Myr}$ period, both the cooling time and dynamical time of the gas around the same region are larger than or equal to the period, so the effect is approximately the same as a continuous jet.

The jet with visible duty cycles ('Th6e44-B4- ${ }_{d} 100$ ') has a weaker effect than a continuous jet with the same average flux ('Th6e43B4-m2e-1') most likely owing to threshold effects. The continuous 

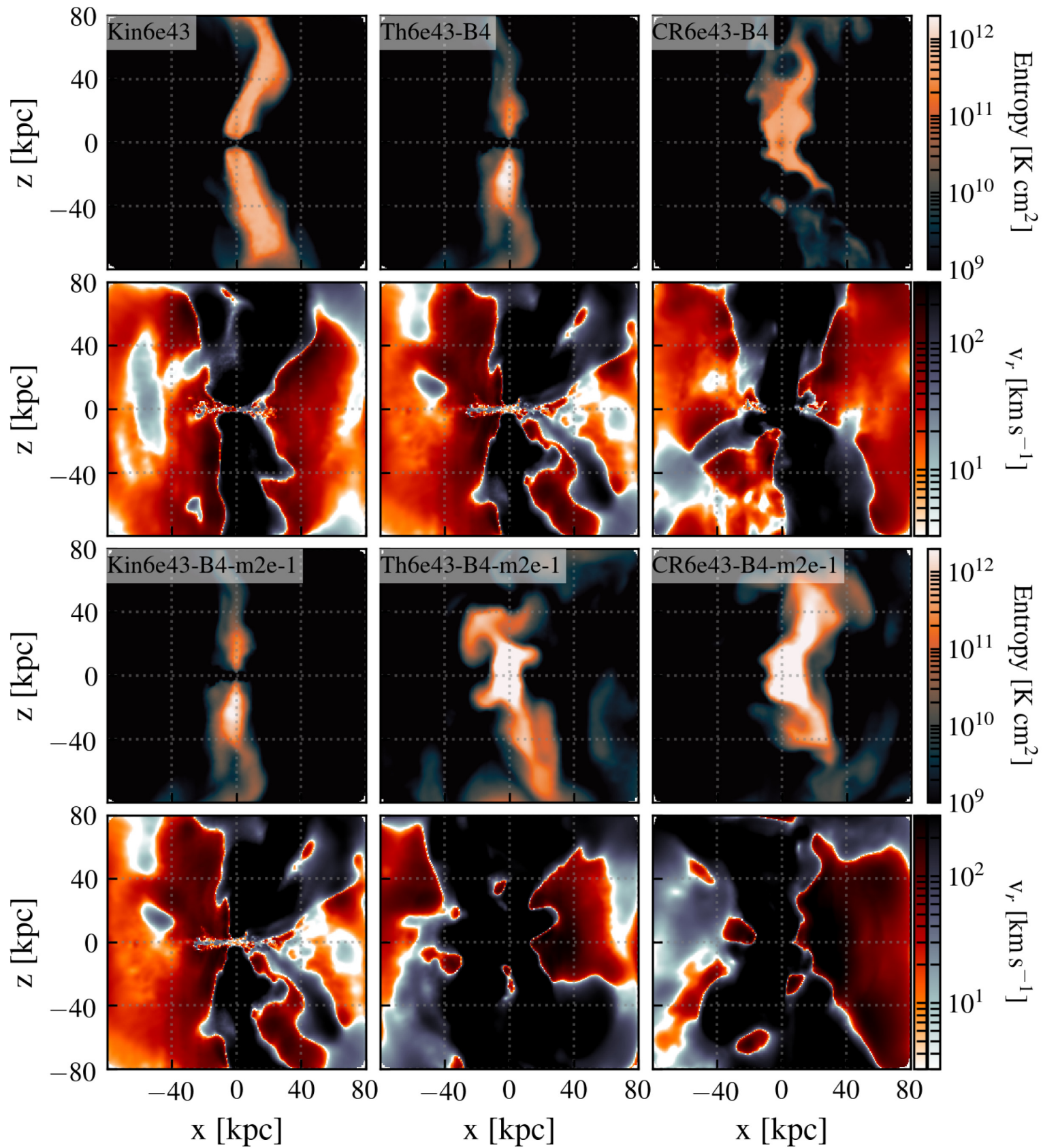

Figure 15. Entropy and radial velocity morphology for runs with different mass fluxes while keeping the same energy flux, shown in a $\delta y=10 \mathrm{kpc}$ slice. With the same thermal or CR energy flux of $6 \times 10^{43} \mathrm{erg} \mathrm{s}^{-1}$, a run with lower mass flux but higher energy per unit mass results in a wider jet cocoon. The situation is not as evident in the cases with lower energy thermal jets or kinetic jets.

run with $10 \times$ higher energy ('Th6e44-B4') quenches similarly to the continuous run ('Th6e43-B4-m2e-1'), so increasing the flux in the 'on' state does not increase the efficiency of quenching dramatically. This is likely due in part to the fact that the perpendicular expansion of the cocoon is sub-linear in time and $\dot{E}$. Additionally, since cooling times are not much longer than $100 \mathrm{Myr}$, some of the injected energy is lost in each 'off' cycle.

\subsection{Jet precession}

We experimented with different precession angles and precession periods and find that the dominant effect of the precessing kinetic jet is still shock heating the surrounding gas and suppressing the inflows or pushing the gas outward within a specific solid angle. Thus, making an otherwise narrow cocoon 'efficient' requires precession angles $\gtrsim 30-45^{\circ}$ so that the cocoon can become effectively quasiisotropic.

Specifically, we see that when the precession period is around $10 \mathrm{Myr}$, the jet becomes more effective only after the precession angle reaches $\gtrsim 45^{\circ}$ ('Kin6e43-pr45-t $10^{\prime}$ '), where the SFR is lower (see Fig. 1) and the cocoon becomes effectively wider than 'Kin6e43' (Fig. 17). When the period is $100 \mathrm{Myr}$, a slightly smaller $30^{\circ}$ precession ('Kin6e43-pr30-t ${ }_{p} 100$ ') is sufficient to suppress the SFR instead of $45^{\circ}$. Consistently, as shown in Fig. 17, 'Kin6e43-pr30- 


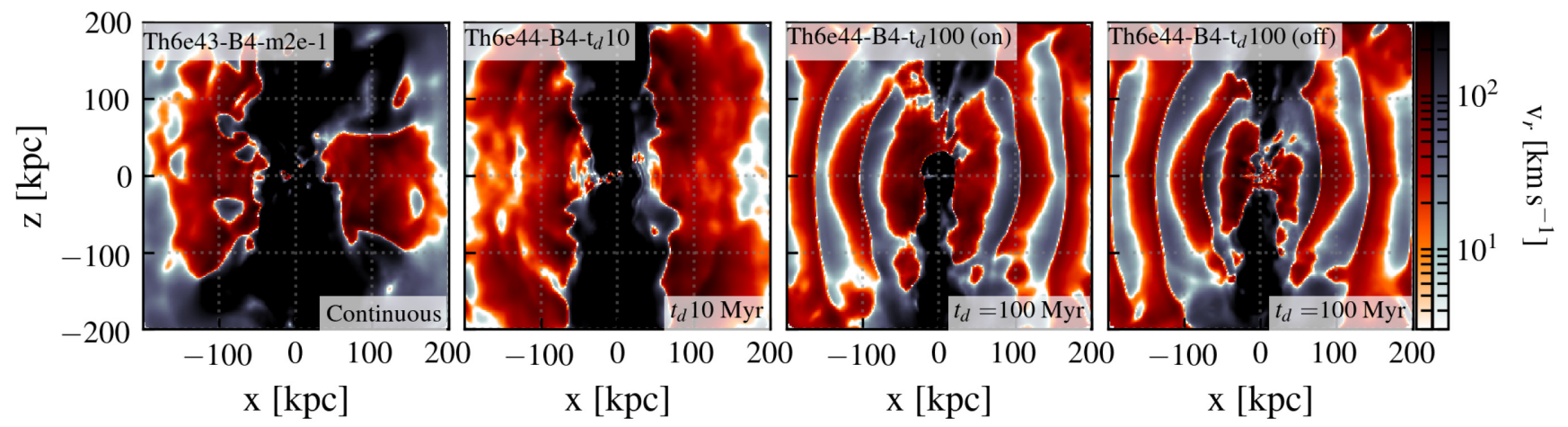

Figure 16. The radial velocity field for thermal jet runs with the same time-averaged energy flux $6 \times 10^{43} \mathrm{erg} \mathrm{s}^{-1}$ within a $\delta y=10 \mathrm{kpc}$ slice, but with different duty cycles (continuous, versus 'on' for $\sim 10$ per cent of the time with a period of 10 or $100 \mathrm{Myr}$, as labeled). The results are shown at the end of each simulation. The run with a $10 \mathrm{Myr}$ period looks effectively similar to the continuous run. Both of them eventually shut down the inflows at the core region of the galaxy completely. The run with a $100 \mathrm{Myr}$ period has concentric shells of inflows and outflows, showing the previous cycles. The inflows at the core region rebuild again when the jet is off.
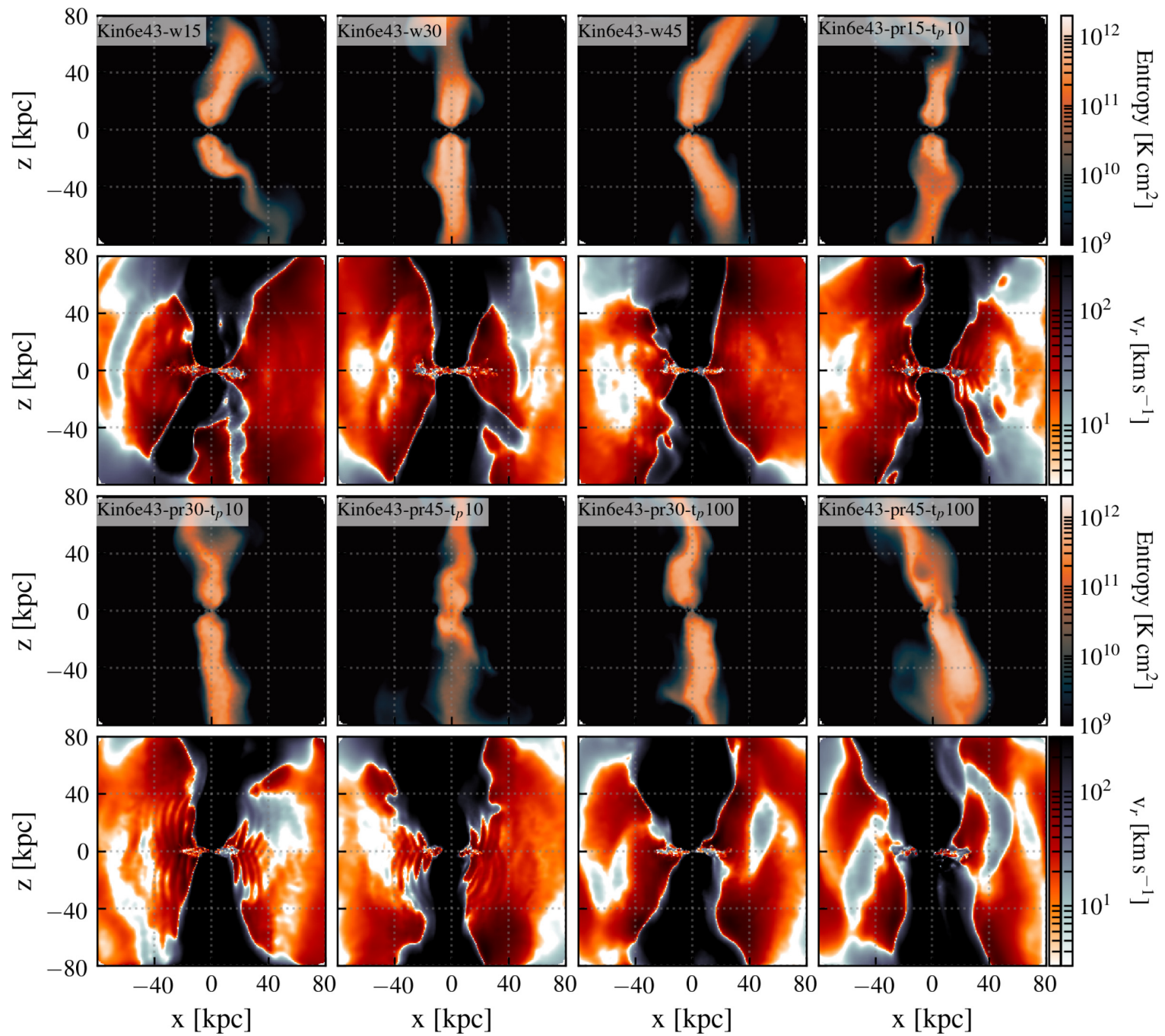

Figure 17. The entropy and radial velocity morphology for runs with different jet opening-angles, precession periods, and precession angles, shown in a $\delta y=$ $10 \mathrm{kpc}$ slice. The runs with a wider opening-angle and/or more extended precession ('Kin6e43-pr30- $\mathrm{t}_{p}$ 100', 'Kin6e43-pr45- $\mathrm{t}_{p}$ 100', 'Kin6e43-pr45- $\mathrm{t}_{p} 10$ ', and 'Kin6e43-w45') have a slightly wider solid angle for outflowing material at the core region of the galaxy, consistent with their lower cooling flows and SFR. 

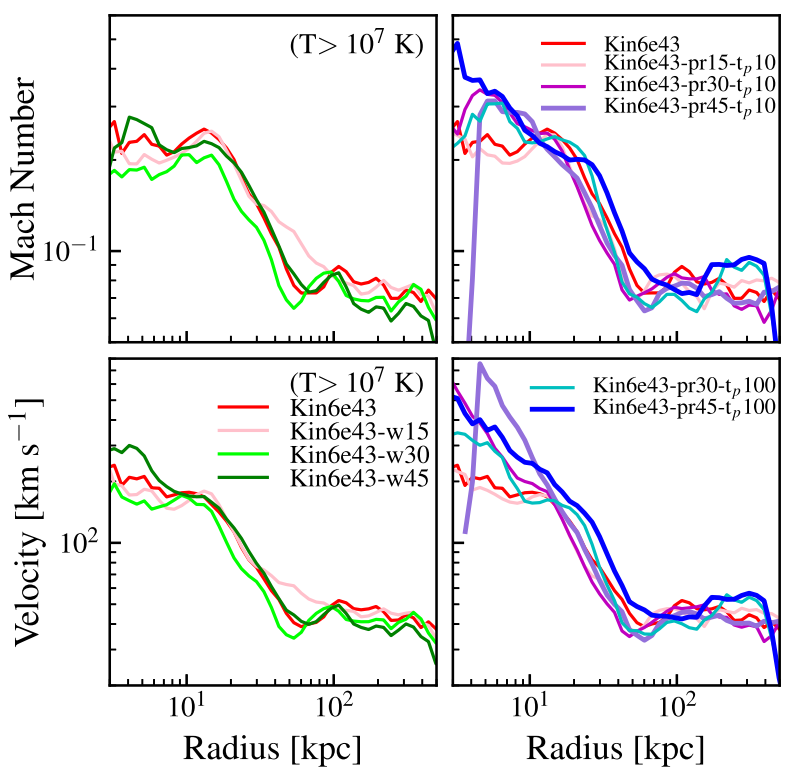

Figure 18. As Fig. 6, $1 \mathrm{D}$ rms Mach number $\left(v_{\text {turb }}^{\theta, \phi} / \sqrt{2} v_{\text {thermal }}\right.$; top), and dispersion $\left(v_{\text {turb }}^{\theta, \phi} / \sqrt{2}\right.$; below $)$ in gas with $T>10^{7} \mathrm{~K}$, averaged over the last $50 \mathrm{Myr}$ of the runs, as a function of radius for the runs with different widths, precession periods, and precession angles from Fig. 17. (We exclude the radial motion to obtain a rough upper bound on the turbulent velocity.) Jet models with a long precession period and a sufficiently wide precession angle can slightly boost the turbulent velocity at $10-70 \mathrm{kpc}$ by a factor of $\leqslant 2$, indicating more efficient turbulent 'stirring'.

$\mathrm{t}_{p} 100$ ' has a wider 'effective' solid angle than 'Kin6e43-pr30- $\mathrm{t}_{p} 10$ ' in the core region.

A non-precessing wide jet ('Kin6e43-w15', 'Kin6e43-w30', and 'Kin6e43-w45') can be viewed as a high-speed precessing jet, since the spawned cells are sampling the opening-angle, with an effective period « Myr. Consistent with the cocoon behaviour discussed above, although the SFR starts to drop when the opening-angle reaches $45^{\circ}$, 'Kin6e43-w45' has a higher SFR and stronger cooling flows than the precessing jet with $45^{\circ}$ precession angle. Therefore, an opening-angle between $45^{\circ}$ and isotropic should be required to reach a similar level of quenching effect.

We also see a factor of $\lesssim 2$ boost in the turbulent velocity at $\sim 10-70 \mathrm{kpc}$ in the runs with a $100 \mathrm{Myr}$ precession period when the opening-angle reaches $30-40^{\circ}$ (Fig. 18). So precessing jets can stir some turbulence, but this, by itself, is nowhere near the level of turbulence required to quench (see Paper II).

The reason for these 'second order' trends is that a more slowly precessing jet can more efficiently expand to a sufficiently large radius when all other jet parameters are equal. Since it stays in each direction for a longer time, it 'clears out' a path before moving to another direction.

\section{COMPARISONS AND CAVEATS}

\subsection{Comparison with Paper II}

In Paper II ( $\mathrm{Su}$ et al. 2020), we considered the same ICs, with AGN toy models using four different mechanisms: CR injection, thermal heating, radial momentum injection, and turbulent stirring with varied $\dot{E}$ and radial distribution of the injection (i.e. these did not follow any physical propagation model). The CR-dominated jets here produce broadly similar results to the simpler CR-injection runs in Paper II, as the CR-dominated cocoon becomes quasi-isotropic and dominates the dynamics. The thermal jets here are qualitatively different from the thermal heating runs in Paper II: here, extremely hot low-density slow-cooling plasma is injected, which inflates a quasi-isotropic cocoon, providing pressure and bouyancy support to halt/reverse inflows inside $R_{\text {cool }}$. In Paper II, the 'thermal heating' was applied as a direct heating term to the pre-existing gas in the galaxy or halo: this requires far more energy to offset cooling 'directly' (since it requires re-heating dense cool-phase gas where cooling is rapid) and is far more unstable, as either the applied heating is less than cooling (in which case the gas still cools) or is greater than cooling (in which case a Sedov-Taylor-type explosion immediately results in 'overheating'). The kinetic jet models here are also distinct from, although in some ways in-between, the momentum-injection and turbulent-stirring runs from Paper II. Our widest-angle kinetic jets are somewhat akin to the isotropic radial momentum injection runs in Paper II, but less explosive owing to the cocoon dynamics that occur here (and not in Paper II owing to the isotropy and effectively large mass-loading of the coupling). None of our models here produces turbulent power approaching the level identified in Paper II as required for quenching from 'pure turbulence' effects.

\subsection{Comparison with other simulations}

We place our results in context through several comparisons as follows (although there is too much previous work to make the comparison comprehensive):

The fiducial model in Li \& Bryan (2014a, b) has the energy flux equally distributed between thermal and kinetic energy. They also tested various jet models with a varied balance between kinetic and thermal energy and different efficiencies. They found, like us, that feedback stronger than the favoured value causes overheating in the core region. They also found that with a pure kinetic model, the cocoons are narrower and less pressurized. However, they suggested that the exact kinetic fraction versus thermal fraction does not significantly alter galaxy evolution, while we found that thermal jets are more effective in quenching. This likely owes to the fact that they do not fix the jet energies: in their runs, when a specific jet model is not as efficient in quenching at the same energy flux, the accretion rate and energy flux rise to compensate. Kinetic jets are also shown to be able to quench the galaxy, reach selfregulation, and maintain the $\mathrm{CC}$ properties across a range of halo mass in Gaspari et al. (2011a, b, 2012a) and Gaspari, Ruszkowski \& Sharma (2012b). This probably occurs for the same reason discussed above, and also the frequently higher jet velocity in their model.

In Bourne \& Sijacki (2017), the authors discussed the CGM turbulence caused by kinetic jets and compared with the CGM turbulence caused by substructures. The authors found that the jet is mostly responsible for the smaller scale turbulence around its edges, but not as effective in inducing larger-scale turbulence. We find a qualitatively similar result that even our most widely precessing jets boost the turbulence at a large radius at most by a factor of 2 , insufficient to quench on its own (Paper II). We also found that a more widely precessing jet can heat the core region up more, consistent with the widely re-oriented jet in Cielo et al. (2018).

Cosmic ray jets have been studied by Wang, Ruszkowski \& Yang (2020; in a $4 \times 10^{13} \mathrm{M}_{\odot}$ halo), Ruszkowski et al. (2017b), and Yang, Gaspari \& Marlow (2019) in a more massive Perseus-mass cluster. The CR energy flux we find to stably quench a $10^{14} \mathrm{M}_{\odot}$ halo $\left(\sim 6 \times 10^{42} \sim \sim 6 \times 10^{43} \mathrm{erg} \mathrm{s}^{-1}\right)$ in this work is roughly consistent with the energy range suggested in Wang et al. (2020), given their 
slightly less massive system. Also consistent with Yang et al. (2019), we find that a CR-dominated jet is generally more efficient at quenching and results in a wider bubble compared to a kinetic jet. Like Ruszkowski et al. (2017b), we find that CR pressure plays a key role regulating the cooling flows, and that including CR motion relative to the gas is the key. Otherwise, if CRs were purely advected, they would behave explosively like thermal energy. Note that we parametrized the $\mathrm{CR}$ motion relative to the gas as diffusion, but within our approximations, this is mathematically identical to their super-Alfvén streaming.

\subsection{Possible further observational probes}

To further constrain the models here, a more detailed analysis of X-ray properties and further comparisons with the multiphase observations will be required. We leave this for future work, but briefly comment on directions we think would be fruitful. Although various models in this work are broadly consistent with the $\mathrm{X}$ ray inferred radially averaged density, temperature, and entropy profiles, the detailed spatial distribution of these properties may vary, especially between the region closer to and further from the jet axis. These can be further constrained by more extensive X-ray map comparisons. Likewise mapping the kinetic properties (inflow/outflow and turbulent velocities versus polar angle) near the jet can further constrain the models. Given that the gas properties at very large radii are dominated by the initial conditions in isolated galaxy simulations, thermal and kinetic Sunyaev-Zeldovich (Sunyaev \& Zeldovich 1970) properties might not be as sensitive to the jet model. As shown in Fig. 12, the thermal properties of lower temperature gas can differ within $\sim 30 \mathrm{kpc}$ between runs with $\mathrm{CR}$ and thermal jets of different energy flux. These will predict different column densities of various ions in different phases.

We have verified that, in our $\mathrm{CR}$ jets, the predicted $\sim \mathrm{GeV}$ gamma-ray luminosity from hadronic interactions is below the current observational upper limits (Ackermann et al. 2016; Wiener \& Zweibel 2019). 'CR6e43' and 'CR6e43-B4-m2e-1' have $L_{\gamma} \sim$ $1-3 \times 10^{41} \mathrm{erg} \mathrm{s}^{-1}$. In 'CR6e42' $L_{\gamma}$ grows from $\sim 10^{41} \mathrm{erg} \mathrm{s}^{-1}$ to $\sim 1-2 \times 10^{42} \mathrm{erg} \mathrm{s}^{-1}$, roughly at the upper limit. The values in the latter case are higher due to the denser core that develops. Likewise, the estimated $\sim \mathrm{GHz}$ radio luminosity from secondary $\mathrm{CR}$ electrons is well within the observational constraint from the radio flux assuming all the secondary CR electrons decay via synchrotron emission (e.g. Giacintucci et al. 2014; Bravi et al. 2016).

\subsection{Limitation of our models}

We emphasize that we are, by design, testing jet models with constant flux in a fixed initial cluster configuration. Our model does not include dynamically variable black hole accretion, so is not 'selfregulating'. Although we explore the effect of duty cycles, we expect a self-consistently fueled jet will have a more complicated duty cycle. With these limitations in mind, the less dramatic 'overheated' models we considered (like 'Th6e43-B4-m2e-1' or 'Kin-6e43-iso') may be allowed if the jet only lasts for a shorter duration. The most overheated models like 'Kin6e44' or 'Th6e44', on the other hand, may still result in tension with observations even if the jet is on only for a short time episodically, like what we see in our duty cycle test 'Th6e44-B4- $t_{d} 100$ '. Moreover, among the surveyed parameter space, fewer non-overheated quiescent runs stay within the $\mathrm{CC}$ range, while a wider variety of them would be classified as NCC. The lower-energy CR jets 'CR-6e42' fall the most robustly in the former case, while the entropy and density profiles of the other runs have some NCC features to some extent. Therefore, to account for both the observed CC and NCC populations, cycles between the two populations should be expected, and further study of this possibility, including simulations with live black hole accretion, will be done in future work.

Another limitation of this work is the lack of a cosmological environment in our simulations. For example, there are no satellites/substructures in our halo, which can alter the large scale turbulence and other ICM gas properties. Moreover, we observed limited X-ray luminosity variation amongst our runs because we start our isolated galaxy simulation from an initial condition resembling a $\mathrm{CC}$ cluster and run it for only $<1.5 \mathrm{Gyr}$. In cosmological simulations, the inclusion of AGN feedback can be even more crucial to reproduce the X-ray luminosity and other thermodynamic properties (e.g. McCarthy et al. 2010; Le Brun et al. 2014; Planelles et al. 2014; Choi et al. 2015; Liang et al. 2016; Henden et al. 2018; Davé et al. 2019).

We also note that we try to test each form of energy flux broadly within the plausible range instead of attempting to match any specific theoretically or observationally motivated model for the energy composition at the jet launching scale. Finally, our experiments are limited because we use a Newtonian MHD code, and cannot consider truly relativistic jets like those in black hole scale simulations.

\section{CONCLUSIONS}

In this paper, we have attempted a systematic exploration of different AGN jet models that inject energy into massive haloes, quenching galaxies and suppressing cooling flows. We specifically considered models with pure kinetic jets, thermal energy dominated jets, and CR jets. We also systematically varied the mass loading, jet width, jet magnetic field strength and field geometry, precession angle and period, and jet duty cycle. These were studied in full-haloscale but non-cosmological simulations including radiative heating and cooling, self-gravity, star formation, and stellar feedback from supernovae, stellar mass-loss, and radiation, enabling a truly 'live' response of star formation and the multiphase ISM to cooling flows. We used a hierarchical super-Lagrangian refinement scheme to reach $\sim 10^{4} \mathrm{M}_{\odot}$ mass resolution, much higher than many previous global studies.

We summarize our key results in the following points and in Table 3:

(i) All our successful models quench via the (initially narrow) jet inflating a quasi-isotropic (large solid-angle at $r \lesssim R_{\text {cool }}$ ) cocoon in which pressure (ram or thermal or CR) is able to balance gravity and 'loft' and heat gas within most of the solid angle inside $R_{\text {cool }}$. Narrowangle cocoons fail to quench regardless of energetics, as inflow continues near the midplane. We stress that the mode with which the jet delivers energy is important and it is not enough to simply directly dump in thermal energy or induce turbulent dissipation to offset cooling. The qualitative behaviours of these cocoons in our kinetic + thermal + magnetic + CR runs are well-described by simple similarity solutions (Sections 4.1 and 4.4).

(ii) This implies three necessary criteria for quenching (suppressing SFR to lower than $3 \mathrm{M}_{\odot} \mathrm{yr}^{-1}$ ) via AGN jets, which we find are sufficient to identify all our quenched runs. (1) A mean energy input rate sufficient to reverse the cooling flow dynamics (sustain pressure that balances gravity), $\left\langle\dot{E}_{\text {tot, } \mathrm{J}}\right\rangle \gtrsim 3 \times$ $10^{43} \mathrm{erg} \mathrm{s}^{-1}\left(\dot{M}_{\text {cool }} / 100 \mathrm{M}_{\odot} \mathrm{yr}^{-1}\right)\left(v_{c}\left[R_{\text {cool }}\right] / 500 \mathrm{~km} \mathrm{~s}^{-1}\right)^{2}$. (2) A specific energy of jet material large enough that the direct (for thermal/CR) or post-shock (for kinetic) cocoon cooling time is 
Table 3. Executive summary of our experiments with different jet models in a $10^{14} \mathrm{M}_{\odot}$ halo.

\begin{tabular}{|c|c|c|c|c|c|}
\hline Form & Variations & $\dot{E}_{q}$ at $2 \mathrm{M}_{\odot} \mathrm{yr}^{-1}$ & Other criteria & Higher $\dot{E} / \dot{m}$ & Problem \\
\hline Thermal & $\begin{array}{c}\text { Constant } \dot{E} \\
10 \% \text { duty cycle }\end{array}$ & $\begin{array}{c}\sim 6 \mathrm{e} 43 \\
\langle\dot{E}(t)\rangle=6 \mathrm{e} 44\end{array}$ & $\begin{array}{c}T_{\text {jet material }} \gtrsim 10^{9} \mathrm{~K} \\
t_{\mathrm{d}} \gtrsim 100 \mathrm{Myr}\end{array}$ & $\begin{array}{l}\text { Wider cocoon } \\
\text { Not tested }\end{array}$ & $\begin{array}{c}\text { Narrow } \dot{E} \text { range } \\
d T / d r<0 \text { when 'on' }\end{array}$ \\
\hline Magnetic & Constant $\mathbf{B}_{\text {ini }}$ & None & None quenches & Wider cocoon & Inefficient by itself \\
\hline
\end{tabular}

This is a summary of all the parameter space we explore. Each column is as follow: (1) 'Form': The dominant energy form in the jet at launch. (2) 'Variations': Qualitative model variations we considered. (3) $\dot{E}_{\mathrm{q}}$ at $2 \mathrm{M}_{\odot} \mathrm{yr}^{-1}$ : The required energy flux to stably quench the galaxy (for our default IC) when the mass flux is $2 \mathrm{M}_{\odot} \mathrm{yr}^{-1}$. (4) 'Other criteria': Additional requirements for this model group to stably quench. $\theta_{\text {op }}$ : opening-angle. $\theta_{\mathrm{p}}$ : precessing angle. (5) Higher $\dot{E} / \dot{m}$ : Effect of increased specific energy in the jet. (6) 'Problems': Physical problems or major qualitative inconsistencies with observations common to all runs in a given 'group'.

always much longer than the cocoon expansion time, $\dot{E}_{\text {tot, J }} / \dot{M}_{\mathrm{J}} \gtrsim$ $10^{17} \mathrm{erg} \mathrm{g}^{-1}$ (e.g. $T>10^{9} \mathrm{~K}$, for thermal jets, or $v \gtrsim 5000 \mathrm{~km} \mathrm{~s}^{-1}$ for kinetic). (3) A means to ensure the cocoon can expand to fill broad solid angles (so effectively suppress inflows) before the jet breaks through $\sim R_{\text {cool }}$. This can be accomplished by either (i) the jet having a dominant fraction of its injection energy in non-kinetic (thermal, CR, or magnetic) form (with the relevant solid angle scaling as $\left.\left(\dot{E}_{\text {tot, jet }} / \dot{E}_{\text {kin, J }}\right)^{3 / 2}\right)$; (ii) an extremely 'light' kinetic jet having a high specific-energy at $\sim$ $10 \mathrm{pc}$ (our coupling radius), with jet velocity at this radius $\gtrsim$ $10^{4}\left(\dot{M}_{\mathrm{J}} / \mathrm{M}_{\odot} \mathrm{yr}^{-1}\right)\left(v_{\mathrm{ff}} / 300 \mathrm{~km} \mathrm{~s}^{-1}\right)\left(\dot{M}_{\mathrm{cool}} / 100 \mathrm{M}_{\odot} \mathrm{yr}^{-1}\right)^{-1}$; or (iii) a large kinetic jet opening or precession angle.

(iii) For thermal+kinetic+magnetic jets (provided the above conditions are met), the criterion for this quenching to become 'explosive' is a larger mean $\left\langle\dot{E}_{\text {tot }}\right\rangle$ by only a factor $\sim 10$ or so. Beyond this energy flux, the jet violently expels the inner halo gas, leaving a remnant which is too hot and has an inverted temperature/entropy gradient compared to observations. Modifying precession or opening-angles or duty cycles can shift the 'preferred' energies slightly but does not appreciably widen this range. Thus there is a rather narrow range of energetics where such jets quench without violating observations. But it remains possible that jets 'selfregulate' to this range in models where accretion and jet power scale self-consistently with nuclear gas properties.

(iv) For CR-dominated jets, the fact that CRs can diffuse or stream through the gas provides a sort of 'pressure valve', making the induced outflows less 'overheated' at high energies. At lower energies, the combination of efficient $\mathrm{CR}$ diffusion isotropizing the $\mathrm{CR}$ cocoon, efficient CR pressure support of cool gas, and the modified nature of thermal instability in a CR-pressure-dominated medium allows CR jets to quench at order-of-magnitude lower energetics. Together this means the allowed dynamic range of energetics for CR-dominated jets is much larger (factor 100 ). Moreover, the lower-energy CR jets are the only successfully quenched models in this category that do not strongly alter the core density and therefore retain observed $\mathrm{CC}$ features and lower turbulent velocities $\lesssim 100 \mathrm{~km} \mathrm{~s}^{-1}$.

Among the surveyed parameter space, few quenched runs always stay within the $\mathrm{CC}$ range. Most of the non-overheated quenched runs evolve toward having NCC entropy and density profiles. Therefore, to account for both the observed CC and NCC populations, cycles between the two populations should be expected. Further study of this possibility, including simulations with jet feedback based on the amount of gas actually accreted will be done in future work.

In summary, our study supports the idea that quenching - at least of observed $z \sim 0$ massive haloes - can be accomplished within the viable parameter space of AGN jets. But with this study and Paper II, we show the viable parameter space that produces successful quenching and does not violate observational constraints is rather narrow, and points to specific jet/cocoon processes and quite possibly a role for CRs. Many caveats remain (see Section 5.4) to explore in future work, alongside more detailed comparisons with observations (Section 5.3).

\section{ACKNOWLEDGEMENTS}

We thank Eliot Quataert for useful discussion. KS acknowledges financial support from the Simons Foundation. We thank Lucy Reading-Ikkanda for preparing the summarizing cartoon Figure (Fig. 7). Support for PFH and co-authors was provided by an Alfred P. Sloan Research Fellowship, NSF Collaborative Research Grant \#1715847, and CAREER grant \#1455342, and NASA grants NNX15AT06G, JPL 1589742, 17-ATP17-0214. GLB acknowledges financial support from the NSF (grant AST-1615955, OAC-1835509, AST-2006176) and computing support from NSF XSEDE. RSS, $\mathrm{CCH}$, and DAA were supported by the Simons Foundation through the Flatiron Institute. DAA was supported by NSF grant AST2009687. CAFG was supported by NSF through grants AST1715216 and CAREER award AST-1652522; by NASA through grant 17-ATP17-0067; and by a Cottrell Scholar Award and a Scialog Award from the Research Corporation for Science Advancement. RF acknowledges financial support from the Swiss National Science Foundation (grant no 194814). DK was supported by NSF grant AST-1715101 and the Cottrell Scholar Award from the Research Corporation for Science Advancement. TKC is supported by Science and Technology Facilities Council (STFC) astronomy consolidated grant ST/T000244/1. Numerical calculations were run on the Flatiron Institute cluster 'popeye' and 'rusty', Caltech cluster 'Wheeler', allocations from XSEDE TG-AST120025, TG-AST130039, and PRAC NSF.1713353 supported by the NSF, and NASA HEC SMD16-7592. This work was carried out as part of the FIRE project and in collaboration with the SMAUG collaboration. SMAUG gratefully acknowledges support from the Center for Computational Astrophysics at the Flatiron Institute, which is supported by the Simons Foundation. 


\section{DATA AVAILABILITY STATEMENT}

The data supporting the plots within this article are available on reasonable request to the corresponding author. A public version of the GIZMOcode is available at http://www.tapir.caltech.edu/ $\sim$ phopkin s/Site/GIZMO.html.

\section{REFERENCES}

Aalto S. et al., 2016, A\&A, 590, A73

Ackermann M. et al., 2016, ApJ, 819, 149

Anderson M. E., Gaspari M., White S. D. M., Wang W., Dai X., 2015, MNRAS, 449, 3806

Anglés-Alcázar D., Faucher-Giguère C.-A., Quataert E., Hopkins P. F., Feldmann R., Torrey P., Wetzel A., Kereš D., 2017, MNRAS, 472, L109

Angles-Alcazar D. et al., 2020, preprint (arXiv:2008.12303)

Babul A., Sharma P., Reynolds C. S., 2013, ApJ, 768, 11

Baldry I. K., Glazebrook K., Brinkmann J., Ivezić Ž., Lupton R. H., Nichol R. C., Szalay A. S., 2004, ApJ, 600, 681

Balogh M. L., Babul A., Voit G. M., McCarthy I. G., Jones L. R., Lewis G. F., Ebeling H., 2006, MNRAS, 366, 624

Banerjee N., Sharma P., 2014, MNRAS, 443, 687

Barai P., Viel M., Murante G., Gaspari M., Borgani S., 2014, MNRAS, 437, 1456

Beck A. M., Lesch H., Dolag K., Kotarba H., Geng A., Stasyszyn F. A., 2012, MNRAS, 422, 2152

Beck R., Brandenburg A., Moss D., Shukurov A., Sokoloff D., 1996, ARA\&A, 34, 155

Begelman M. C., 2004, in Ho L. C., ed., Coevolution of Black Holes and Galaxies. Cambridge Univ. Press, Cambridge, p. 374

Begelman M. C., Cioffi D. F., 1989, ApJ, 345, L21

Begelman M. C., Blandford R. D., Rees M. J., 1980, Nature, 287, 307

Bell E. F., McIntosh D. H., Katz N., Weinberg M. D., 2003, ApJS, 149, 289

Berezinsky V., Gazizov A., Grigorieva S., 2006, Phys. Rev. D, 74, 043005

Binney J., Cowie L. L., 1981, ApJ, 247, 464

Bîrzan L., Rafferty D. A., McNamara B. R., Wise M. W., Nulsen P. E. J., 2004, ApJ, 607, 800

Blandford R. D., Znajek R. L., 1977, MNRAS, 179, 433

Blandford R., Meier D., Readhead A., 2019, ARA\&A, 57, 467

Blanton M. R., Eisenstein D., Hogg D. W., Schlegel D. J., Brinkmann J., 2005, ApJ, 629, 143

Booth C. M., Schaye J., 2009, MNRAS, 398, 53

Bourne M. A., Sijacki D., 2017, MNRAS, 472, 4707

Bravi L., Gitti M., Brunetti G., 2016, preprint (arXiv:1603.00368)

Butsky I. S., Quinn T. R., 2018, ApJ, 868, 108

Butsky I. S., Fielding D. B., Hayward C. C., Hummels C. B., Quinn T. R., Werk J. K., 2020, ApJ, 903, 77

Chan T. K., Kereš D., Hopkins P. F., Quataert E., Su K. Y., Hayward C. C., Faucher-Giguère C. A., 2019, MNRAS, 488, 3716

Choi E., Ostriker J. P., Naab T., Johansson P. H., 2012, ApJ, 754, 125

Choi E., Ostriker J. P., Naab T., Oser L., Moster B. P., 2015, MNRAS, 449, 4105

Cielo S., Babul A., Antonuccio-Delogu V., Silk J., Volonteri M., 2018, A\&A, 617, A58

Ciotti L., Ostriker J. P., 2001, ApJ, 551, 131

Ciotti L., Ostriker J. P., Proga D., 2009, ApJ, 699, 89

Conroy C., van Dokkum P. G., Kravtsov A., 2015, ApJ, 803, 77

Croton D. J. et al., 2006, MNRAS, 365, 11

Davé R., Anglés-Alcázar D., Narayanan D., Li Q., Rafieferantsoa M. H., Appleby S., 2019, MNRAS, 486, 2827

Dekel A., Birnboim Y., 2006, MNRAS, 368, 2

Dekel A., Sari R., Ceverino D., 2009, ApJ, 703, 785

Di Matteo T., Springel V., Hernquist L., 2005, Nature, 433, 604

Dubois Y., Devriendt J., Slyz A., Teyssier R., 2010, MNRAS, 409, 985

Dubois Y., Pichon C., Devriendt J., Silk J., Haehnelt M., Kimm T., Slyz A., 2013, MNRAS, 428, 2885

Dunn R. J. H., Fabian A. C., Sanders J. S., 2006, MNRAS, 366, 758
Eisenreich M., Naab T., Choi E., Ostriker J. P., Emsellem E., 2017, MNRAS, 468,751

Enßlin T., Pfrommer C., Miniati F., Subramanian K., 2011, A\&A, 527, A99

Fabian A. C., 1999, MNRAS, 308, L39

Fabian A. C., 2012, ARA\&A, 50, 455

Fabian A. C., Arnaud K. A., Bautz M. W., Tawara Y., 1994, ApJ, 436, L63

Fabian A. C., Voigt L. M., Morris R. G., 2002, MNRAS, 335, L71

Farber R., Ruszkowski M., Yang H.-Y. K., Zweibel E. G., 2018, ApJ, 856, 112

Faucher-Giguère C.-A., Quataert E., 2012, MNRAS, 425, 605

Feldmann R., Mayer L., 2015, MNRAS, 446, 1939

Fujita Y., Ohira Y., 2011, ApJ, 738, 182

Fujita Y., Kimura S., Ohira Y., 2013, MNRAS, 432, 1434

Gaspari M., Sạdowski A., 2017, ApJ, 837, 149

Gaspari M., Melioli C., Brighenti F., D’Ercole A., 2011a, MNRAS, 411, 349

Gaspari M., Brighenti F., D'Ercole A., Melioli C., 2011b, MNRAS, 415, 1549

Gaspari M., Brighenti F., Temi P., 2012a, MNRAS, 424, 190

Gaspari M., Ruszkowski M., Sharma P., 2012b, ApJ, 746, 94

Giacintucci S., Markevitch M., Venturi T., Clarke T. E., Cassano R., Mazzotta P., 2014, ApJ, 781, 9

Glines F. W., O'Shea B. W., Voit G. M., 2020, preprint (arXiv:2004.00021)

Guan X., Li H., Li S., 2014, ApJ, 781, 48

Guo F., Oh S. P., 2008, MNRAS, 384, 251

Häring N., Rix H.-W., 2004, ApJ, 604, L89

Hawley J. F., Villiers J. D., 2004, Prog. Theor. Phys. Suppl., 155, 132

Henden N. A., Puchwein E., Shen S., Sijacki D., 2018, MNRAS, 479, 5385

Hernquist L., 1990, ApJ, 356, 359

Hickox R. C., Alexander D. M., 2018, ARA\&A, 56, 625

Hillel S., Soker N., 2017, ApJ, 845, 91

Hillel S., Soker N., 2018, Res. Astron. Astrophys., 18, 081

Hitomi Collaboration, 2016, Nature, 535, 117

Hitomi Collaboration, 2018, PASJ, 70, 9

Hopkins P. F., 2015, MNRAS, 450, 53

Hopkins P. F., 2016, MNRAS, 462, 576

Hopkins P. F., 2017, MNRAS, 466, 3387

Hopkins P. F., Elvis M., 2010, MNRAS, 401, 7

Hopkins P. F., Raives M. J., 2016, MNRAS, 455, 51

Hopkins P. F., Hernquist L., Cox T. J., Di Matteo T., Martini P., Robertson B., Springel V., 2005, ApJ, 630, 705

Hopkins P. F., Hernquist L., Cox T. J., Di Matteo T., Robertson B., Springel V., 2006a, ApJS, 163, 1

Hopkins P. F., Hernquist L., Cox T. J., Robertson B., Springel V., 2006b, ApJS, 163, 50

Hopkins P. F., Hernquist L., Cox T. J., Robertson B., Krause E., 2007, ApJ, 669,45

Hopkins P. F., Hernquist L., Cox T. J., Kereš D., 2008, ApJS, 175, 356

Hopkins P. F., Hernquist L., Hayward C. C., Narayanan D., 2012, MNRAS, 425,1121

Hopkins P. F., Narayanan D., Murray N., 2013, MNRAS, 432, 2647

Hopkins P. F., Kereš D., Oñorbe J., Faucher-Giguère C.-A., Quataert E., Murray N., Bullock J. S., 2014, MNRAS, 445, 581

Hopkins P. F., Torrey P., Faucher-Giguère C.-A., Quataert E., Murray N., 2016, MNRAS, 458, 816

Hopkins P. F. et al., 2018a, MNRAS, 477, 1578

Hopkins P. F. et al., 2018b, MNRAS, 480, 800

Hopkins P. F. et al., 2019, MNRAS, 492, 2993

Hopkins P. F., Chan T. K., Ji S., Hummels C. B., Kereš D., Quataert E., Faucher-Giguère C.-A., 2021a, MNRAS, 501, 3640

Hopkins P. F., Chan T. K., Squire J., Quataert E., Ji S., Kereš D., FaucherGiguère C.-A., 2021b, MNRAS, 501, 3663

Hopkins P. F., Squire J., Chan T. K., Quataert E., Ji S., Kereš D., FaucherGiguère C.-A., 2021c, MNRAS, 501, 4184

Hudson D. S., Mittal R., Reiprich T. H., Nulsen P. E. J., Andernach H., Sarazin C. L., 2010, A\&A, 513, A37

Humphrey P. J., Buote D. A., 2013, MNRAS, 436, 2879

Humphrey P. J., Buote D. A., Brighenti F., Flohic H. M. L. G., Gastaldello F., Mathews W. G., 2012, ApJ, 748, 11 
Jacob S., Pfrommer C., 2017a, MNRAS, 467, 1449

Jacob S., Pfrommer C., 2017b, MNRAS, 467, 1478

Jacob S., Pakmor R., Simpson C. M., Springel V., Pfrommer C., 2018, MNRAS, 475, 570

Ji S. et al., 2020, MNRAS, 496, 4221

Johansson P. H., Naab T., Burkert A., 2009, ApJ, 690, 802

Kaastra J. S., Mewe R., Nieuwenhuijzen H., 1996, in Yamashita K., Watanabe T., eds, UV and X-ray Spectroscopy of Astrophysical and Laboratory Plasmas. p. 411

Kauffmann G. et al., 2003, MNRAS, 341, 54

Kereš D., Katz N., Weinberg D. H., Davé R., 2005, MNRAS, 363, 2

Kereš D., Katz N., Davé R., Fardal M., Weinberg D. H., 2009, MNRAS, 396, 2332

Kim D.-W., Fabbiano G., 2013, ApJ, 776, 116

Kim W.-T., Narayan R., 2003, ApJ, 596, L139

Kroupa P., 2002, Science, 295, 82

Le Brun A. M. C., McCarthy I. G., Schaye J., Ponman T. J., 2014, MNRAS, 441,1270

Leitherer C. et al., 1999, ApJS, 123, 3

Lense J., Thirring H., 1918, Phys. Z., 19, 156

Li Y., Bryan G. L., 2014a, ApJ, 789, 54

Li Y., Bryan G. L., 2014b, ApJ, 789, 153

Li Y., Bryan G. L., Ruszkowski M., Voit G. M., O’Shea B. W., Donahue M., 2015, ApJ, 811, 73

Li Y., Ruszkowski M., Bryan G. L., 2017, ApJ, 847, 106

Liang L., Durier F., Babul A., Davé R., Oppenheimer B. D., Katz N., Fardal M., Quinn T., 2016, MNRAS, 456, 4266

Li Y.-P. et al., 2018, ApJ, 866, 70

Lodders K., 2003, ApJ, 591, 1220

McCarthy I. G. et al., 2010, MNRAS, 406, 822

McDonald M., Veilleux S., Mushotzky R., 2011, ApJ, 731, 33

McDonald M. et al., 2013, ApJ, 774, 23

McKinney J. C., Tchekhovskoy A., Bland ford R. D., 2012, MNRAS, 423, 3083

McNamara B. R., Nulsen P. E. J., 2007, ARA\&A, 45, 117

Madgwick D. S., Somerville R., Lahav O., Ellis R., 2003, MNRAS, 343, 871

Martí-Vidal I., Marcaide J. M., Alberdi A., Pérez-Torres M. A., Ros E., Guirado J. C., 2011, A\&A, 533, A111

Martig M., Bournaud F., Teyssier R., Dekel A., 2009, ApJ, 707, 250

Martizzi D., Quataert E., Faucher-Giguère C.-A., Fielding D., 2019, MNRAS, 483, 2465

Mernier F. et al., 2017, A\&A, 603, A80

Mittal R., Hudson D. S., Reiprich T. H., Clarke T., 2009, A\&A, 501, 835

Navarro J. F., Frenk C. S., White S. D. M., 1996, ApJ, 462, 563

Nixon C. J., King A. R., Price D. J., 2012a, MNRAS, 422, 2547

Nixon C., King A., Price D., Frank J., 2012b, ApJ, 757, L24

O’Dea C. P. et al., 2008, ApJ, 681, 1035

Ostriker J. P., Choi E., Ciotti L., Novak G. S., Proga D., 2010, ApJ, 722, 642

Parrish I. J., Quataert E., Sharma P., 2010, ApJ, 712, L194

Parrish I. J., McCourt M., Quataert E., Sharma P., 2012, MNRAS, 419, L29

Pellegrini S., Ciotti L., Negri A., Ostriker J. P., 2018, ApJ, 856, 115

Peres C. B., Fabian A. C., Edge A. C., Allen S. W., Johnstone R. M., White D. A., 1998, MNRAS, 298, 416

Peterson J. R., Fabian A. C., 2006, Phys. Rep., 427, 1

Pfrommer C., 2013, ApJ, 779, 10

Pfrommer C., Pakmor R., Schaal K., Simpson C. M., Springel V., 2017, MNRAS, 465, 4500

Pillepich A. et al., 2018, MNRAS, 473, 4077

Planelles S., Borgani S., Fabjan D., Killedar M., Murante G., Granato G. L., Ragone-Figueroa C., Dolag K., 2014, MNRAS, 438, 195

Pozzetti L. et al., 2010, A\&A, 523, A13

Prasad D., Sharma P., Babul A., 2015, ApJ, 811, 108

Pringle J. E., 1996, MNRAS, 281, 357

Pringle J. E., 1997, MNRAS, 292, 136

Rafferty D. A., McNamara B. R., Nulsen P. E. J., 2008, ApJ, 687, 899

Reiprich T. H., Böhringer H., 2002, ApJ, 567, 716

Ressler S. M., Quataert E., Stone J. M., 2018, MNRAS, 478, 3544

Reynolds C. S., Balbus S. A., Schekochihin A. A., 2015, ApJ, 815, 41
Richings A. J., Faucher-Giguère C.-A., 2018a, MNRAS, 474, 3673 Richings A. J., Faucher-Giguère C.-A., 2018b, MNRAS, 478, 3100

Ruszkowski M., Oh S. P., 2010, ApJ, 713, 1332

Ruszkowski M., Yang H.-Y. K., Zweibel E., 2017a, ApJ, 834, 208

Ruszkowski M., Yang H.-Y. K., Reynolds C. S., 2017b, ApJ, 844, 13

Sanderson A. J. R., Ponman T. J., O'Sullivan E., 2006, MNRAS, 372, 1496

Sanderson A. J. R., O'Sullivan E., Ponman T. J., 2009, MNRAS, 395, 764

Schure K. M., Kosenko D., Kaastra J. S., Keppens R., Vink J., 2009, A\&A, 508,751

Sharma P., Parrish I. J., Quataert E., 2010, ApJ, 720, 652

Sharma P., McCourt M., Quataert E., Parrish I. J., 2012, MNRAS, 420, 3174

Sijacki D., Springel V., Di Matteo T., Hernquist L., 2007, MNRAS, 380, 877

Silk J., Rees M. J., 1998, A\&A, 331, L1

Soker N., Sarazin C. L., 1990, ApJ, 348, 73

Somerville R. S., Hopkins P. F., Cox T. J., Robertson B. E., Hernquist L., 2008, MNRAS, 391, 481

Springel V., 2000, MNRAS, 312, 859

Springel V., White S. D. M., 1999, MNRAS, 307, 162

Springel V., Di Matteo T., Hernquist L., 2005, MNRAS, 361, 776

Stanek R., Evrard A. E., Böhringer H., Schuecker P., Nord B., 2006, ApJ, 648,956

Stern J., Fielding D., Faucher-Giguère C.-A., Quataert E., 2019, MNRAS, 488,2549

Strong A. W., Moskalenko I. V., 1998, ApJ, 509, 212

Strong A. W., Porter T. A., Digel S. W., Jóhannesson G., Martin P., Moskalenko I. V., Murphy E. J., Orlando E., 2010, ApJ, 722, L58

Su Y., White R. E. III, Miller E. D., 2013, ApJ, 775, 89

Su Y., Buote D., Gastaldello F., Brighenti F., 2015, ApJ, 805, 104

Su K.-Y., Hopkins P. F., Hayward C. C., Faucher-Giguère C.-A., Kereš D., Ma X., Robles V. H., 2017, MNRAS, 471, 144

Su K.-Y. et al., 2019, MNRAS, 487, 4393 (Paper I)

Su K.-Y. et al., 2020, MNRAS, 491, 1190 (Paper II)

Sunyaev R. A., Zeldovich Y. B., 1970, Ap\&SS, 7, 3

Tamura T. et al., 2001, A\&A, 365, L87

Tchekhovskoy A., Narayan R., McKinney J. C., 2011, MNRAS, 418, L79

Torrey P. et al., 2020, MNRAS, 497, 5292

Trotta R., Jóhannesson G., Moskalenko I. V., Porter T. A., Ruiz de Austri R., Strong A. W., 2011, ApJ, 729, 106

Tucker W. H., Rosner R., 1983, ApJ, 267, 547

Uhlig M., Pfrommer C., Sharma M., Nath B. B., Enßlin T. A., Springel V., 2012, MNRAS, 423, 2374

Vernaleo J. C., Reynolds C. S., 2006, ApJ, 645, 83

Voigt L. M., Fabian A. C., 2004, MNRAS, 347, 1130

Voigt L. M., Schmidt R. W., Fabian A. C., Allen S. W., Johnstone R. M., 2002, MNRAS, 335, L7

Voit G. M., Donahue M., Bryan G. L., McDonald M., 2015, Nature, 519, 203

Voit G. M., Meece G., Li Y., O’Shea B. W., Bryan G. L., Donahue M., 2017, ApJ, 845, 80

Wang C., Ruszkowski M., Yang H. Y. K., 2020, MNRAS, 493, 4065

Weinberger R. et al., 2017a, MNRAS, 465, 3291

Weinberger R., Ehlert K., Pfrommer C., Pakmor R., Springel V., 2017b, MNRAS, 470, 4530

Weinberger R. et al., 2018, MNRAS, 479, 4056

Werner N. et al., 2013, ApJ, 767, 153

Wetzel A. R., Tinker J. L., Conroy C., 2012, MNRAS, 424, 232

White C. J., Stone J. M., Quataert E., 2019, ApJ, 874, 168

Wiener J., Zweibel E. G., 2019, MNRAS, 488, 280

Wiener J., Oh S. P., Guo F., 2013, MNRAS, 434, 2209

Yang H.-Y. K., Reynolds C. S., 2016a, ApJ, 818, 181

Yang H. Y. K., Reynolds C. S., 2016b, ApJ, 829, 90

Yang H. Y. K., Sutter P. M., Ricker P. M., 2012, MNRAS, 427, 1614

Yang H. Y. K., Gaspari M., Marlow C., 2019, ApJ, 871, 6

Yoon D., Yuan F., Gan Z.-M., Ostriker J. P., Li Y.-P., Ciotti L., 2018, ApJ, 864,6

Zakamska N. L., Narayan R., 2003, ApJ, 582, 162

Zhuravleva I. et al., 2014, Nature, 515, 85

ZuHone J. A., Markevitch M., Zhuravleva I., 2016, ApJ, 817, 110 


\section{APPENDIX A: DENSITY AND ENTROPY PROFILES FOR ALL RUNS}

In Figs A1 and A2 we provide the density and luminosity-weighted entropy profiles of all our runs averaged over the last $\sim 50 \mathrm{Myr}$. For the overheated runs, we plotted the averaged profiles from
550-600 Myr to compare on an equal footing. The runs labeled 'overheated' in Table 2 generally have very low density high entropy core regions within $\sim 500 \mathrm{Myr}$. The runs labeled 'strong CF' or 'slight $\downarrow$ ' in Table 2 generally have an overdense core region. The other runs agree more reasonably with the observations.
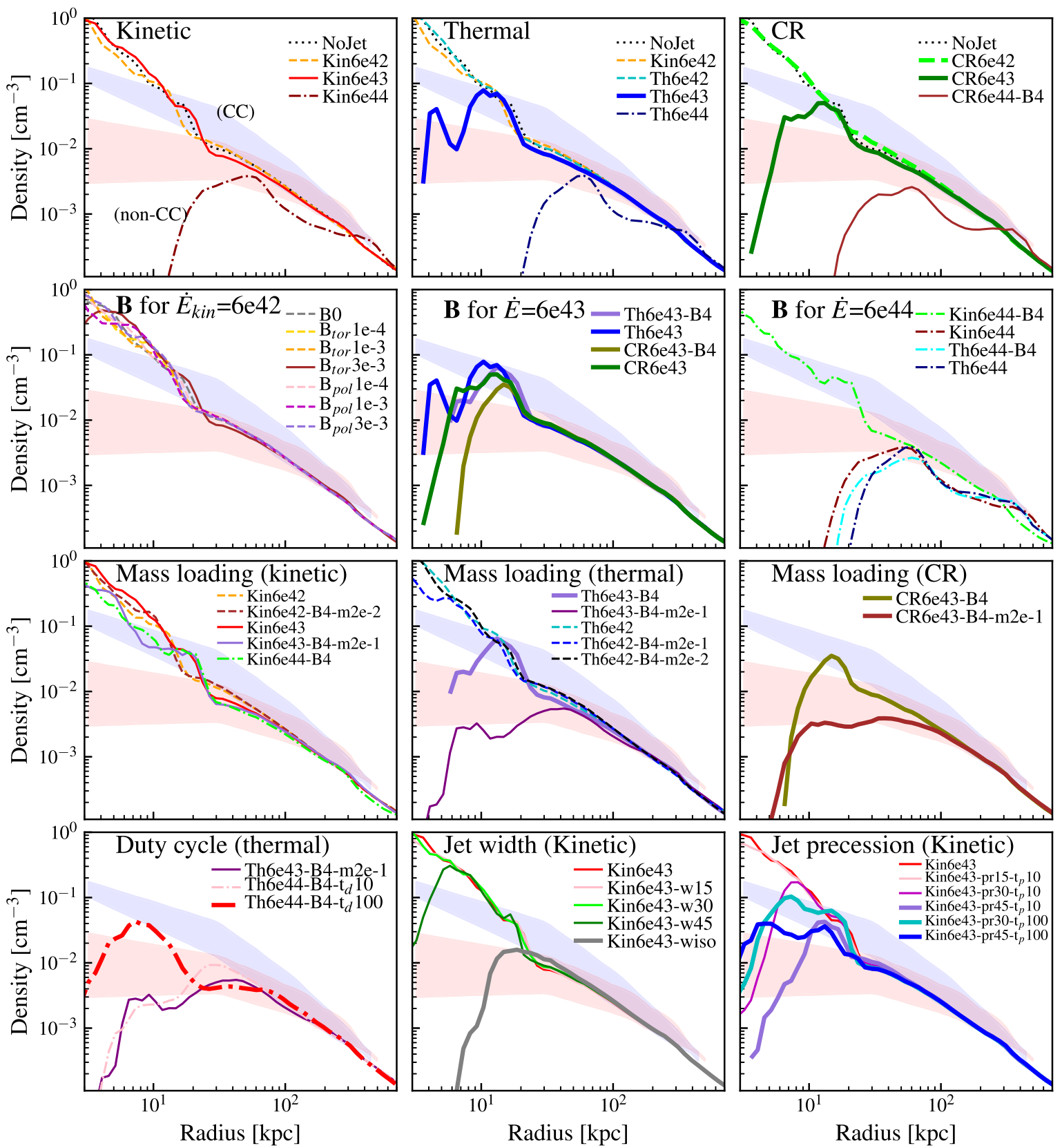

Figure A1. Density versus radius averaged over the last $\sim 50 \mathrm{Myr}$ in the all runs from Fig. 1. For the overheated runs, we plotted the averaged profiles from 550 to $600 \mathrm{Myr}$ to compare on an equal footing. The shaded regions indicate the observational density profiles (scaled) for CC (blue) and NCC (red) clusters (McDonald et al. 2013), scaled according to the halo mass differences. Runs labeled 'overheated' in Table 2 generally have very low density core regions. Runs labeled 'strong CF' or 'slight $\downarrow$ ' in Table 2 generally have an overdense core region. The other runs agree reasonably well with the observations. 

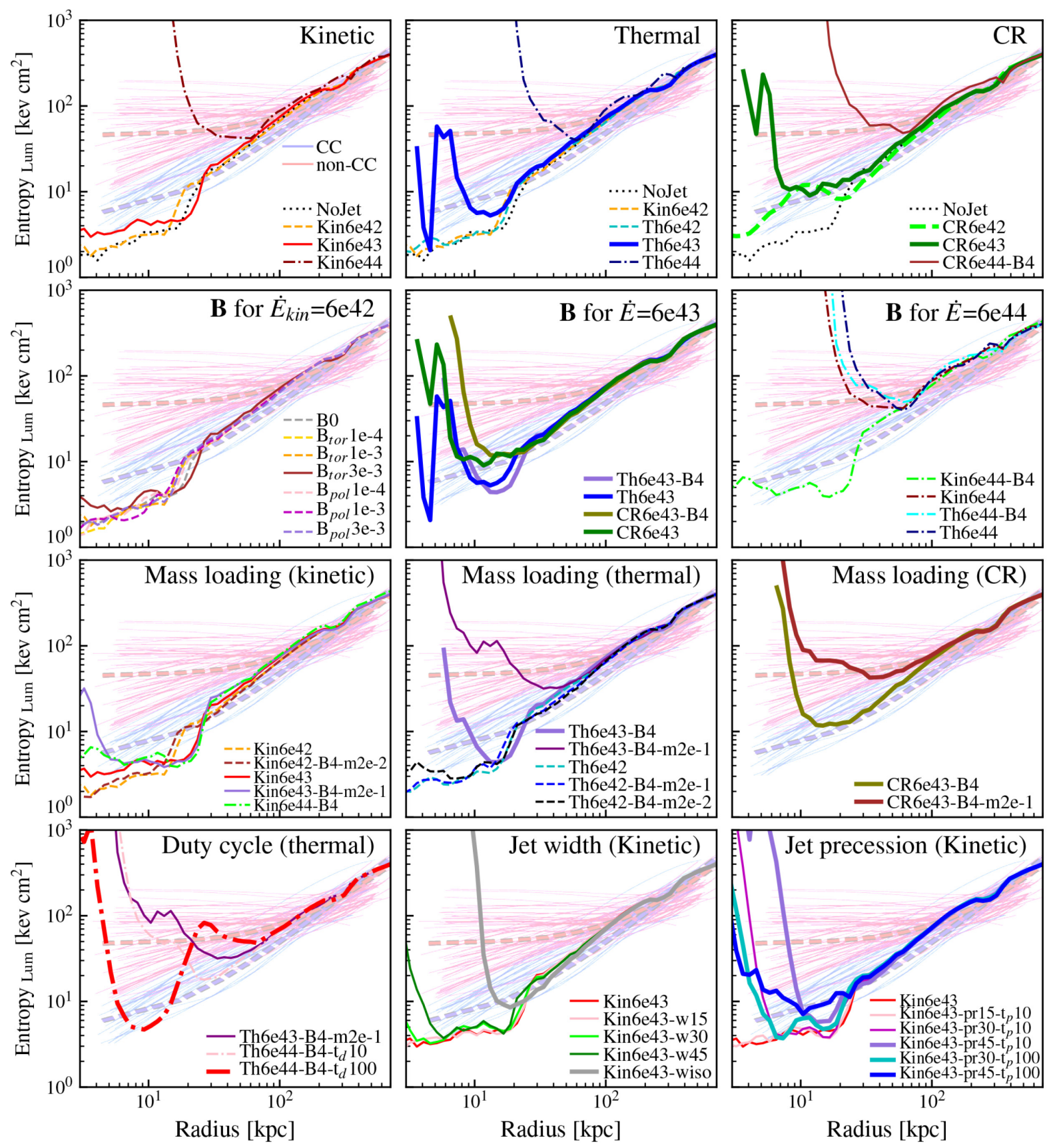

Figure A2. Luminosity-weighted entropy versus radius averaged over the last $\sim 50 \mathrm{Myr}$ in the all runs from Fig. 1. For the overheated runs, we plotted the averaged profiles from 550 to $600 \mathrm{Myr}$ to compare on an equal footing. The light curves in the bottom row indicate the observational entropy profiles (scaled) for CC (blue) and NCC (red) clusters (McDonald et al. 2013; scaled according to the halo mass differences). Runs labeled 'overheated' in Table 2 generally have very high entropy in the core regions. The other runs have entropy profiles qualitatively resembling the observed CC populations.

\section{APPENDIX B: THERMAL STABILITY FOR CR JETS WITH DIFFERENT FLUX}

CRs stabilize the gas more effectively in the runs with lower CR energy injection. The reason is due to the balance between CR energy and thermal energy, $f_{\mathrm{CR}}=P_{\mathrm{CR}} / P_{\text {thermal }}$. The gas will only follow an isochoric and constant thermal+CR pressure process when CRs are the dominant energy form (high $f_{\mathrm{CR}}$ ).

The first row of Fig. B1 shows such a ratio of the two CR injection runs at the beginning (100 Myr) and end (1.5 Gyr) of the simulations. In 'CR6e42', initially, the ratio, $f_{\mathrm{CR}}$, for the gas that is cooling (the blue square region in Fig. B1) is not sufficiently high, so the density in that phase shows a broader distribution resembling that in the 'Th6e42' run. After the CR energy builds up as the energy injection continues and $f_{\mathrm{CR}}$ increases, the density distribution becomes narrow. On the other hand, in the higher CR flux run, 'CR6e43', initially, the ratio $f_{\mathrm{CR}}$ is slightly higher than the 'CR6e 42 ' run. However, at a later time, the CR energy of the gas with density $n>10^{-2} \mathrm{~cm}^{-3}$ does not increase much due to gas expansion (because of the suppressed density) and the advection of CR-rich gas. Instead, the CR energy goes into the lower density phase at larger radii (the red square region in Fig. B1). 

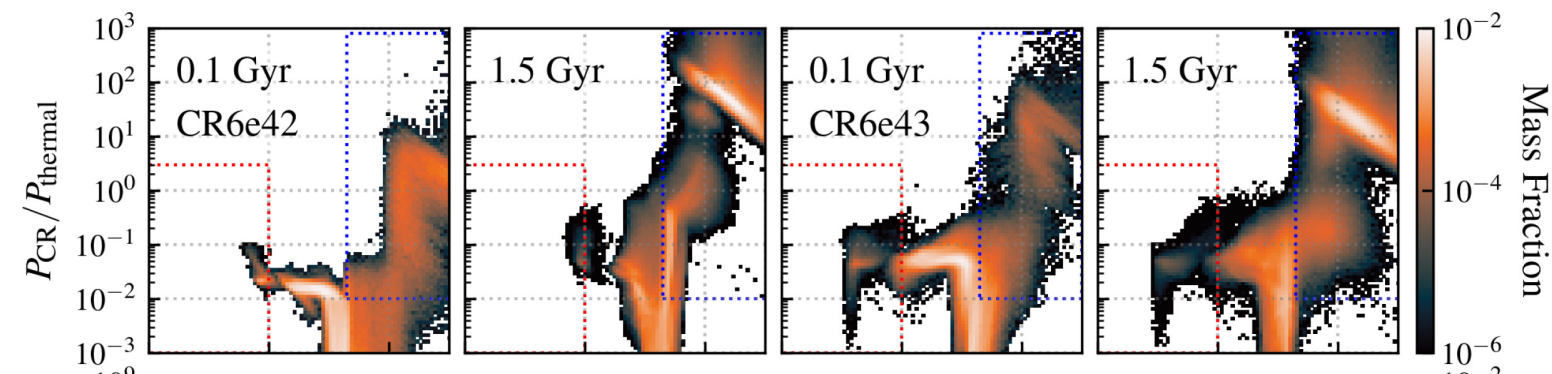

\section{3
0
0
0
0
0
0
0}
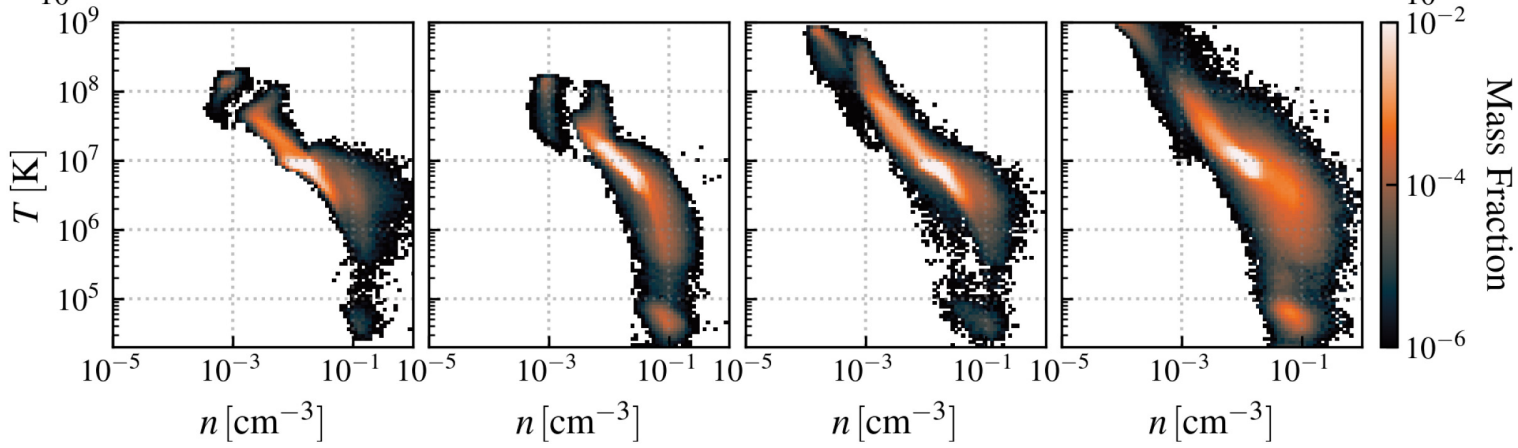

Figure B1. The distribution of $P_{\mathrm{CR}} / P_{\text {thermal }}$ as a function of gas density (top), and the phase distribution in the temperature-density plane (bottom) for the 'CR6e42' and 'CR6e43' runs at $100 \mathrm{Myr}$ and $1.5 \mathrm{Gyr}$, for the gas above $10^{4} \mathrm{~K}$ and from 10 to $30 \mathrm{kpc}$. At $1.5 \mathrm{Gyr}$, the run with a lower CR flux jet ('CR6e42') reaches a higher $P_{\mathrm{CR}} / P_{\text {thermal }}$ than the run with higher CR flux ('CR6e43') for the gas that is cooling (the region outlined with a blue dotted line in the plot). The latter run has more CRs distributed to the lower density phase at a larger radius (the red region in the plot). Only when $P_{\mathrm{CR}} / P_{\text {thermal }}$ builds up to a sufficiently high value does the density distribution tighten.

This paper has been typeset from a TEX/LTTEX file prepared by the author. 